\title{
Model Theory for Process Algebra
}

\author{
J.A. Bergstra ${ }^{1,2}$ and C.A. Middelburg ${ }^{3}$ \\ 1 Programming Research Group, University of Amsterdam, \\ P.O. Box 41882, 1009 DB Amsterdam, the Netherlands \\ janb@science.uva.nl \\ 2 Department of Philosophy, Utrecht University, \\ P.O. Box 80126, 3508 TC Utrecht, the Netherlands \\ janb@phil.uu.nl \\ 3 Computing Science Department, Eindhoven University of Technology, \\ P.O. Box 513, 5600 MB Eindhoven, the Netherlands \\ keesm@win.tue.nl
}

\begin{abstract}
We present a first-order extension of the algebraic theory about processes known as ACP and its main models. Useful predicates on processes, such as deadlock freedom and determinism, can be added to this theory through first-order definitional extensions. Model theory is used to analyse the discrepancies between identity in the models of the first-order extension of ACP and bisimilarity of the transition systems extracted from these models, and also the discrepancies between deadlock freedom in the models of a first-order definitional extension of this theory and deadlock freedom of the transition systems extracted from these models. First-order definitions are material to the formalization of an interpretation of one theory about processes in another. We give a comprehensive example of such an interpretation too.
\end{abstract}

Keywords: model theory, process algebra, first-order theory, full bisimulation models, bisimilarity, reachability, deadlock freedom, determinism, interpretation.

1998 CR Categories: D.2.4, F.1.2, F.3.1, F.4.1.

\section{Introduction}

Usually, theories about processes such as ACP $[12,7]$ and CCS $[26,27]$ are equationally axiomatized. However, it is also possible to give first-order theories. An important advantage of a first-order approach is that it makes available the tool of first-order definition of predicates and operations on processes.

In this paper, we present a first-order extension of ACP and its main models. The first-order extension concerned includes a binary reachability predicate on processes with an associated first-order axiom schema for subprocess induction. The reachability predicate can be used to give first-order definitions of many general properties of processes, such as deadlock freedom and determinism, and the axiom schema for subprocess induction can then be used to verify whether processes have these properties. This is one of the interesting applications of first-order definitions of predicates on processes. 
First-order definitions of predicates and operations on processes are generally indispensable for the formalization of an interpretation of one theory about processes in another. For example, a first-order definition of the deadlock freedom predicate permits the formalization of the interpretation of $\mathrm{BPA}$ in $\mathrm{BPA}_{\delta}[7]$ (both are subtheories of ACP). By first-order definitions of operations on processes, we are able to formalize more complicated interpretations, such as the interpretation of BPPA $[17,8]$ in the first-order extension of ACP. If one theory is interpretable in another theory, then a model of the former theory can be obtained from each model of the latter theory by taking a submodel of a restriction of an expansion by definitions. The expansion concerns the first-order definable operations on processes needed in the formalization of the interpretation concerned; and the first-order definable predicate on processes needed in the formalization of the interpretation determines the domain of the submodel. This technique to construct models can be regarded as a first-order generalization of the SRM-technique from [2].

In this paper, we analyse the discrepancies between identity in the models of the first-order extension of ACP and external bisimilarity, i.e. bisimilarity of the transition systems extracted from these models. Besides external bisimilarity, we pay attention to observational equivalence; and we have a look at other related issues such as bisimilarity based on structural operational semantics and modal characterization of external bisimilarity. We also analyse the discrepancies between deadlock freedom in the models of a first-order definitional extension of the first-order extension of ACP and external deadlock freedom, i.e. deadlock freedom of the transition systems extracted from these models. Additionally, we briefly consider the comparable discrepancies for determinism.

We give a comprehensive example of the formalization of an interpretation of one theory about processes in another too. It happens that the first-order extension of $\mathrm{BPA}_{\delta}$, which is a subtheory of the first-order extension of ACP, gets great expressive power in case it is extended with restricted reachability predicates. Even the first-order extension of ACP can be interpreted in it. As a matter of fact, the comprehensive example of the formalization of an interpretation concerns this interpretation.

The structure of this paper is as follows. First of all, we introduce $\mathrm{BPA}_{\delta}^{\mathrm{fo}}$, the (finitary) first-order extension of an important subtheory of ACP, to wit $\mathrm{BPA}_{\delta}$ (Section 2). Next, we consider some useful infinitary and second-order axioms (Section 3). After that, we introduce transition systems and bisimilarity of transition systems (Section 4) and full bisimulation models, the main models of $\mathrm{BPA}_{\delta}^{\text {fo }}$ (Section 5). Thereupon, we analyse the discrepancies between external bisimilarity and identity in models of $\mathrm{BPA}_{\delta}^{\text {fo }}$ (Section 6) and investigate the related external equivalence known as observational equivalence (Section 7). Following this, we have a closer look at bisimilarity based on structural operational semantics (Section 8) and the modal characterization of external bisimilarity (Section 9). Then, we extend $\mathrm{BPA}_{\delta}^{\text {fo }}$ with a deadlock freedom predicate and analyse the discrepancies between external deadlock freedom and internal deadlock freedom in models of the extension of $\mathrm{BPA}_{\delta}^{\text {fo }}$ concerned (Section 10). We 
also briefly consider the extension with a determinism predicate (Section 11). After that, we consider the addition of restricted reachability predicates to $\mathrm{BPA}_{\delta}^{\text {fo }}$ (Section 12). Next, we introduce $\mathrm{ACP}^{\text {fo }}$, the first-order extension of ACP (Section 13) and the full bisimulation models of $\mathrm{ACP}^{\text {fo }}$ (Section 14). Thereupon, we consider interpretations of one theory in another (Section 15) and give as an example the interpretation of $\mathrm{ACP}^{\mathrm{fo}}$ in the extension of $\mathrm{BPA}_{\delta}^{\text {fo }}$ with restricted reachability predicates (Section 16). Finally, we make some concluding remarks (Section 17).

\section{The First-Order Theory $\mathrm{BPA}_{\delta}^{\text {fo }}$}

In this section, we present $\mathrm{BPA}_{\delta}^{\mathrm{fo}}$, a first-order extension of an important subtheory of ACP, being known as $\mathrm{BPA}_{\delta}$. In $\mathrm{BPA}_{\delta}^{\text {fo }}$, it is assumed that there is a fixed but arbitrary finite set of actions A with $\delta \notin \mathrm{A}$.

The first-order theory $\mathrm{BPA}_{\delta}^{\text {fo }}$ has the following nonlogical symbols:

- the deadlock constant $\delta$;

- for each $a \in \mathrm{A}$, the action constant $a$;

- the binary alternative composition operator + ;

- the binary sequential composition operator ·;

- the binary summand inclusion predicate symbol $\sqsubseteq$;

- for each $a \in \mathrm{A}$, the unary action termination predicate symbol $\stackrel{a}{\rightarrow} \sqrt{ }$;

- for each $a \in \mathrm{A}$, the binary action step predicate symbol $\stackrel{a}{\rightarrow}$;

- the binary reachability predicate symbol $\rightarrow$.

We use infix notation for the binary operators, postfix notation for the unary predicate symbols and infix notation for the binary predicate symbols. The following precedence conventions are used to reduce the need for parentheses. Operators bind stronger than predicate symbols, and predicate symbols bind stronger than logical connectives and quantifiers. Moreover, the operator · binds stronger than the operator + , the logical connective $\neg$ binds stronger than the logical connectives $\wedge$ and $\vee$, and the logical connectives $\wedge$ and $\vee$ bind stronger than the logical connectives $\Rightarrow$ and $\Leftrightarrow$. Quantifiers are given the smallest possible scope.

The constants and operators of $\mathrm{BPA}_{\delta}^{\text {fo }}$ are the same as the constants and operators of $\mathrm{BPA}_{\delta}$. The additional nonlogical symbols of $\mathrm{BPA}_{\delta}^{\mathrm{fo}}$ are all predicate symbols. In the context of $\mathrm{BPA}_{\delta}$, the summand inclusion predicate symbol is sometimes used in abbreviations for equations expressing summand inclusions. The action termination and action step predicate symbols are used in the description of the structural operational semantics of $\mathrm{BPA}_{\delta}$. That usage is related to the usage in the theory $\mathrm{BPA}_{\delta}^{\mathrm{fo}}$, but the one should not be mistaken for the other. A similar remark applies to the reachability predicate symbol.

Let $t$ and $t^{\prime}$ be closed terms of $\mathcal{L}\left(\mathrm{BPA}_{\delta}^{\text {fo }}\right)$. Intuitively, the constants and operators can be explained as follows:

$-\delta$ cannot perform any action; 
- $a$ first performs action $a$ and then terminates successfully;

$-t+t^{\prime}$ behaves either as $t$ or as $t^{\prime}$, but not both;

$-t \cdot t^{\prime}$ first behaves as $t$, but when $t$ terminates succesfully it continues by behaving as $t^{\prime}$.

Intuitively, the predicates can be explained as follows:

$-t \sqsubseteq t^{\prime}$ means that $t^{\prime}$ is capable of behaving as $t$;

$-t \stackrel{a}{\rightarrow} \vee$ means that $t$ is capable of performing action $a$ and then terminating successfully;

$-t \stackrel{a}{\rightarrow} t^{\prime}$ means that $t$ is capable of performing action $a$ and then behaving as $t^{\prime}$

- $t \rightarrow t^{\prime}$ means that $t$ is capable of performing a number of actions and then behaving as $t^{\prime}$.

The axioms of $\mathrm{BPA}_{\delta}^{\text {fo }}$ are given in Table 1. Many axioms in this table are actually axiom schemas. RDPf and RSPf are axiom schemas in which $t_{1}, \ldots, t_{n}$ $(n \geq 1)$ stand for arbitrary terms of $\mathcal{L}\left(\mathrm{BPA}_{\delta}^{\text {fo }}\right)$ in which only the variables $x_{1}, \ldots, x_{n}$ may have occurrences and all those occurrences are guarded. We call an occurrence of a variable $x$ in a term $t$ guarded if $t$ has a subterm of the form $a \cdot t^{\prime}$ with $t^{\prime}$ containing this occurrence of $x$. The notation $t_{i}\left(t_{1}^{\prime}, \ldots, t_{n}^{\prime}\right)$ is used for the term obtained from $t_{i}$ by replacing all occurrences of $x_{1}, \ldots, x_{n}$ by $t_{1}^{\prime}, \ldots, t_{n}^{\prime}$, respectively. BS and RS are axiom schemas in which $\phi$ stands for an arbitrary formula of $\mathcal{L}\left(\mathrm{BPA}_{\delta}^{\mathrm{fo}}\right)$ in which only the variables $x_{1}$ and $x_{2}$ may have free occurrences. The notation $\phi\left(t_{1}, t_{2}\right)$ is used for the formula obtained from $\phi$ by replacing all free occurrences of $x_{1}$ and $x_{2}$ by $t_{1}$ and $t_{2}$, respectively. SI2SI9, TR1-TR2 and R2 are axiom schemas in which $a$ and $b$ stand for arbitrary actions.

Axioms $\mathrm{A} 1-\mathrm{A} 7$ are the axioms of $\mathrm{BPA}_{\delta}$. So $\mathrm{BPA}_{\delta}^{\text {fo }}$ imports the (equational) axioms of $\mathrm{BPA}_{\delta}$. Axiom schemas RDPf and RSPf are relevant to the use of recursion for describing (potentially) non-terminating processes. They will be explained separately below. Axiom SI1 is the defining axiom of the summand inclusion predicate. Axiom schemas SI2-SI9 exclude models that identify processes that cannot be related by a bisimulation. Axiom SI10 is an extensionality axiom for summand inclusion. The instances of axiom schema TR1 are the defining axioms of the action termination predicates and the instances of axiom schema TR2 are the defining axioms of the action step predicates. Axiom schema BS, called the bisimilarity axiom schema, excludes models that do not identify processes that can be related by a first-order definable bisimulation. Axiom R1 and axiom schemas R2 and RS implicitly define the reachability predicate. Axiom schema RS is an induction schema, called the subprocess induction schema.

We do not claim that the axioms of $\mathrm{BPA}_{\delta}^{\text {fo }}$ are independent. For example, axiom SI2 is derivable from axioms A7 and SI6. Axiom SI10 and axiom schema BS are dependent in a weak sense: extensionality for equality, i.e.

$$
\bigwedge_{a \in \mathrm{A}}((a \sqsubseteq x \Leftrightarrow a \sqsubseteq y) \wedge \forall z \bullet(a \cdot z \sqsubseteq x \Leftrightarrow a \cdot z \sqsubseteq y)) \Rightarrow x=y,
$$

is derivable from SI10 and derivable from BS. 
Table 1. Axioms for $\mathrm{BPA}_{\delta}^{\text {fo }}$ (in $t_{1}, \ldots, t_{n}$ all occurrences of variables must be guarded)

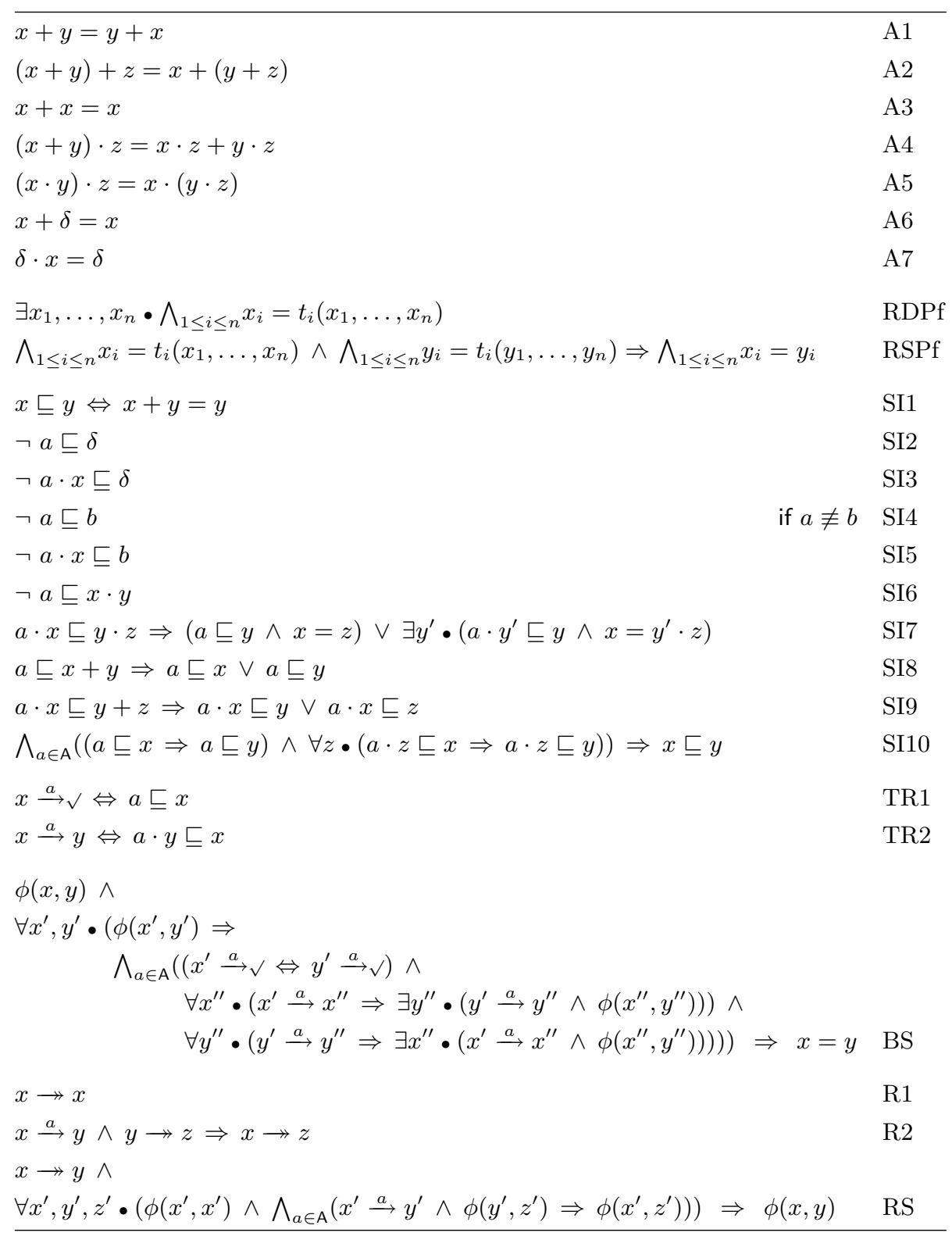

The axiom schemas RDPf an RSPf are called the recursive definition principle and the recursive specification principle for finite guarded recursive specifications. A guarded recursive specification (over $\mathrm{BPA}_{\delta}^{\text {fo }}$ ) is a set of equations $E=\left\{x=t_{x} \mid x \in V\right\}$ where $V$ is a set of variables and each $t_{x}$ is a term 
of $\mathcal{L}\left(\mathrm{BPA}_{\delta}^{\text {fo }}\right)$ in which only the variables in $V$ may have occurrences and all those occurrences are guarded. There is an instance of RDPf and an instance of RSPf for each finite guarded recursive specification $E$. We write $\operatorname{RDPf}^{E}$ for the instance of RDPf for $E$ and $\mathrm{RSPf}^{E}$ for the instance of RDPf for $E$. RDPf $E$ expresses that $E$ has at least one solution and $\operatorname{RSPf}^{E}$ expresses that $E$ has at most one solution.

Because the implications from right to left are derivable, the (outmost) occurrence of " $\Rightarrow$ " in SI7-SI10 and BS can be replaced by " $\Leftrightarrow$ ". The equivalences

$$
\begin{aligned}
& x=y \Leftrightarrow x \sqsubseteq y \wedge y \sqsubseteq x, \\
& x+y \sqsubseteq z \Leftrightarrow x \sqsubseteq z \wedge y \sqsubseteq z .
\end{aligned}
$$

are easily derived from axiom SI1 and axiom SI10, respectively. Both equivalences are used in the proof of Theorem 2.1 (see below).

Using the reachability predicate, we can give explicit definitions of other properties of processes. For example, deadlock freedom, absence of termination, and determinism can be explicitly defined as follows:

$$
\begin{aligned}
& \operatorname{dlf}(x) \Leftrightarrow \neg x \rightarrow \delta, \\
& \operatorname{perp}(x) \Leftrightarrow \neg x \rightarrow \delta \wedge \bigwedge_{a \in \mathrm{A}} \neg x \rightarrow a, \\
& \operatorname{det}(x) \Leftrightarrow \forall y \bullet\left(x \rightarrow y \Rightarrow \bigwedge_{a \in \mathrm{A}}((y \stackrel{a}{\rightarrow} \vee \Rightarrow \forall z \bullet \neg y \stackrel{a}{\rightarrow} z) \wedge\right. \\
&\left.\left.\forall z, z^{\prime} \bullet\left(y \stackrel{a}{\rightarrow} z \wedge y \stackrel{a}{\rightarrow} z^{\prime} \Rightarrow z=z^{\prime}\right)\right)\right) .
\end{aligned}
$$

Using the subprocess induction schema, we can derive a formula according to which case distinction with respect to reachability can be made.

Proposition 2.1 (Case Distinction for Reachability). The following formula is derivable from $\mathrm{BPA}_{\delta}^{\text {fo }}$ :

$$
\begin{aligned}
& x \rightarrow y \Rightarrow \\
& x=y \vee \bigvee_{a \in \mathrm{A}} x \stackrel{a}{\rightarrow} y \vee \exists z \bullet\left(z \neq x \wedge \bigvee_{a \in \mathrm{A}}(x \stackrel{a}{\rightarrow} z \wedge z \rightarrow y)\right) .
\end{aligned}
$$

Proof. We use $\operatorname{cdr}(x, y)$ as an abbreviation for the right-hand side of the above implication. We will apply RS, taking $x \rightarrow y \wedge \operatorname{cdr}(x, y)$ for $\phi$. When we have shown that $x \rightarrow y \Rightarrow(x \rightarrow y \wedge \operatorname{cdr}(x, y))$, we can immediately conclude that $x \rightarrow y \Rightarrow \operatorname{cdr}(x, y)$ and we are done.

It remains to be shown by means of RS that $x \rightarrow y \Rightarrow(x \rightarrow y \wedge \operatorname{cdr}(x, y))$. First of all, we conclude from $\mathrm{R} 1$, because obviously $\operatorname{cdr}(x, x)$, that

$$
\forall x^{\prime} \bullet\left(x^{\prime} \rightarrow x^{\prime} \wedge \operatorname{cdr}\left(x^{\prime}, x^{\prime}\right)\right) .
$$

Moreover, we easily derive the following implications:

$$
x^{\prime} \stackrel{a^{\prime}}{\longrightarrow} y^{\prime} \wedge y^{\prime} \rightarrow z^{\prime} \Rightarrow x^{\prime} \rightarrow z^{\prime},
$$




$$
\begin{aligned}
& x^{\prime} \stackrel{a^{\prime}}{\rightarrow} y^{\prime} \wedge y^{\prime} \rightarrow z^{\prime} \wedge y^{\prime}=z^{\prime} \Rightarrow \bigvee_{a \in \mathrm{A}} x^{\prime} \stackrel{a}{\rightarrow} z^{\prime}, \\
& x^{\prime} \stackrel{a^{\prime}}{\rightarrow} y^{\prime} \wedge y^{\prime} \rightarrow z^{\prime} \wedge \bigvee_{a \in \mathrm{A}} y^{\prime} \stackrel{a}{\rightarrow} z^{\prime} \Rightarrow \\
& \bigvee_{a \in \mathrm{A}} x^{\prime} \stackrel{a}{\rightarrow} z^{\prime} \vee \exists z \bullet\left(z \neq x^{\prime} \wedge \bigvee_{a \in \mathrm{A}}\left(x^{\prime} \stackrel{a}{\rightarrow} z \wedge z \rightarrow z^{\prime}\right)\right), \\
& x^{\prime} \stackrel{a^{\prime}}{\rightarrow} y^{\prime} \wedge y^{\prime} \rightarrow z^{\prime} \wedge \exists z \bullet\left(z \neq y^{\prime} \wedge \bigvee_{a \in \mathrm{A}}\left(y^{\prime} \stackrel{a}{\rightarrow} z \wedge z \rightarrow z^{\prime}\right)\right) \Rightarrow \\
& \quad \exists z \bullet\left(z \neq x^{\prime} \wedge \bigvee_{a \in \mathrm{A}}\left(x^{\prime} \stackrel{a}{\rightarrow} z \wedge z \rightarrow z^{\prime}\right)\right) .
\end{aligned}
$$

The first implication is derived using R2, the second implication is derived elementary, the third implication is derived using R1 and R2 (with distinction between the cases $x^{\prime}=y^{\prime}, y^{\prime}=z^{\prime}$ and $\left.x^{\prime} \neq y^{\prime} \wedge y^{\prime} \neq z^{\prime}\right)$, and the fourth implication is derived elementary (with distinction between the cases $x^{\prime}=y^{\prime}$ and $x^{\prime} \neq y^{\prime}$ ). The left-hand sides of the second, third and fourth implication are conjunctions of $x^{\prime} \stackrel{a^{\prime}}{\longrightarrow} y^{\prime} \wedge y^{\prime} \rightarrow z^{\prime}$ and one of the disjuncts of $\operatorname{cdr}\left(y^{\prime}, z^{\prime}\right)$. The right-hand sides of these implication consists of one or two of the disjuncts of $\operatorname{cdr}\left(x^{\prime}, z^{\prime}\right)$. Hence, we also conclude that

$$
\bigwedge_{a^{\prime} \in \mathrm{A}}^{\forall x^{\prime}, y^{\prime}, z^{\prime} \bullet}\left(x^{\prime} \stackrel{a^{\prime}}{\longrightarrow} y^{\prime} \wedge\left(y^{\prime} \rightarrow z^{\prime} \wedge \operatorname{cdr}\left(y^{\prime}, z^{\prime}\right)\right) \Rightarrow x^{\prime} \rightarrow z^{\prime} \wedge \operatorname{cdr}\left(x^{\prime}, z^{\prime}\right)\right) .
$$

Using the subprocess induction schema, it follows from these conclusions that $x \rightarrow y \Rightarrow(x \rightarrow y \wedge \operatorname{cdr}(x, y))$.

A well-known subtheory of $\mathrm{BPA}_{\delta}$ is $\mathrm{BPA}, \mathrm{BPA}_{\delta}$ without the deadlock constant and consequently without axioms A6 and A7. Analogously, we have a subtheory of $\mathrm{BPA}_{\delta}^{\text {fo }}$, to wit $\mathrm{BPA}^{\text {fo }}$. As to be expected, the first-order theory $\mathrm{BPA}^{\text {fo }}$ is $\mathrm{BPA}_{\delta}^{\text {fo }}$ without the deadlock constant and without axioms A6, A7, SI2 and SI3. In other words, the possibility that a process gets into a deadlock is not covered by $\mathrm{BPA}^{\mathrm{fo}}$. Note that deadlock freedom cannot be defined in $\mathrm{BPA}^{\mathrm{fo}}$.

To prove a statement for all closed terms of $\mathcal{L}\left(\mathrm{BPA}_{\delta}^{\text {fo }}\right)$, it is sufficient to prove it for all basic terms over $\mathrm{BPA}_{\delta}^{\text {fo }}$. The set $\mathcal{B}$ of basic terms over $\mathrm{BPA}_{\delta}^{\text {fo }}$ is inductively defined by the following rules:

$-\delta \in \mathcal{B}$

- if $a \in \mathrm{A}$, then $a \in \mathcal{B}$;

- if $a \in \mathrm{A}$ and $t \in \mathcal{B}$, then $a \cdot t \in \mathcal{B}$;

- if $t_{1}, t_{2} \in \mathcal{B}$, then $t_{1}+t_{2} \in \mathcal{B}$.

We can prove that all closed terms of $\mathcal{L}\left(\mathrm{BPA}_{\delta}^{\text {fo }}\right)$ are derivably equal to a basic term over $\mathrm{BPA}_{\delta}^{\mathrm{fo}}$.

Proposition 2.2 (Elimination). For all closed terms t of $\mathcal{L}\left(\mathrm{BPA}_{\delta}^{\mathrm{fo}}\right)$ there exists a basic term $t^{\prime} \in \mathcal{B}$ such that $\mathrm{BPA}_{\delta}^{\text {fo }} \vdash t=t^{\prime}$. 
Table 2. Infinitary First-Order Axioms

\begin{tabular}{ll}
\hline$\exists x_{1}, x_{2}, \ldots \bullet \bigwedge_{i \geq 1} x_{i}=t_{i}\left(x_{1}, x_{2}, \ldots\right)$ & $\mathrm{RDP}$ \\
$\bigwedge_{i \geq 1} x_{i}=t_{i}\left(x_{1}, x_{2}, \ldots\right) \wedge \bigwedge_{i \geq 1} y_{i}=t_{i}\left(x_{1}, x_{2}, \ldots\right) \Rightarrow \bigwedge_{i \geq 1} x_{i}=y_{i}$ & $\mathrm{RSP}$ \\
\hline
\end{tabular}

Proof. This follows immediately from the elimination property for $\mathrm{BPA}_{\delta}$ : the closed terms of $\mathcal{L}\left(\mathrm{BPA}_{\delta}^{\text {fo }}\right)$ are the same as the closed terms of $\mathcal{L}\left(\mathrm{BPA}_{\delta}\right)$, and the equational axioms of $\mathrm{BPA}_{\delta}^{\text {fo }}$ are the same as the axioms of $\mathrm{BPA}_{\delta}$.

For closed equations, $\mathrm{BPA}_{\delta}^{\text {fo }}$ is a complete theory.

Theorem 2.1 (Complete Theory for Closed Equations). For all closed terms $t_{1}, t_{2}$ of $\mathcal{L}\left(\mathrm{BPA}_{\delta}^{\text {fo }}\right)$, we have either $\mathrm{BPA}_{\delta}^{\text {fo }} \vdash t_{1}=t_{2}$ or $\mathrm{BPA}_{\delta}^{\text {fo }} \vdash \neg t_{1}=t_{2}$, but not both.

Proof. In Section 5, we will show that there exists a model of $\mathrm{BPA}_{\delta}^{\text {fo }}$. From this, it follows by the Extended Completeness Theorem (see e.g. [20]) that there are no closed terms $t_{1}, t_{2}$ of $\mathcal{L}\left(\mathrm{BPA}_{\delta}^{\text {fo }}\right)$ such that both $t_{1}=t_{2}$ and $\neg t_{1}=t_{2}$ are derivable. Moreover, the equivalence $x=y \Leftrightarrow x \sqsubseteq y \wedge y \sqsubseteq x$ is derivable. For these reasons, and Proposition 2.2, it is sufficient to prove that for all basic terms $t_{1}, t_{2} \in \mathcal{B}$, either $\mathrm{BPA}_{\delta}^{\text {fo }} \vdash t_{1} \sqsubseteq t_{2}$ or $\mathrm{BPA}_{\delta}^{\text {fo }} \vdash \neg t_{1} \sqsubseteq t_{2}$. This is easily proved by induction on the sum of the lengths of $t_{1}$ and $t_{2}$. All cases follow immediately from axioms SI1-SI9, except the cases where $t_{1}$ is of the form $t+t^{\prime}$. Those cases follow immediately from the derivable equivalence $x+y \sqsubseteq z \Leftrightarrow x \sqsubseteq z \wedge y \sqsubseteq z$.

\section{Infinitary and Second-Order Axioms}

It appears to be of use to add certain infinitary and second-order axioms to $\mathrm{BPA}_{\delta}^{\mathrm{fo}}$. In this section, we consider those axioms.

The recursive definition principle and recursive specification principle for finite guarded recursive specifications (RDPf and RSPf) do not exclude models in which there are countably infinite guarded recursive specifications without a unique solution. The infinitary axiom schemas RDP and RSP from Table 2 would exclude all such models. Like in the case of axiom schemas RDPf and RSPf, we write $\mathrm{RDP}^{E}$ and $\mathrm{RSP}^{E}$ for the instances of RDP and RSP, respectively, for guarded recursive specification $E$.

The instances of axiom schema RSP are formulas of $\mathcal{L}_{\omega_{1} \omega}\left(\mathrm{BPA}_{\delta}^{\text {fo }}\right)$, the firstorder language of $\mathrm{BPA}_{\delta}^{\mathrm{fo}}$ with conjunctions and disjunctions of countable sets of formulas. The instances of axiom schema RDP are formulas of $\mathcal{L}_{\omega_{1} \omega_{1}}\left(\mathrm{BPA}_{\delta}^{\mathrm{fo}}\right)$, the first-order language of $\mathrm{BPA}_{\delta}^{\text {fo }}$ with conjunctions and disjunctions of countable sets of formulas and quantification on countable sets of variables. RDP and RSP are not axiomatizable in the usual finitary first-order language $\mathcal{L}\left(\mathrm{BPA}_{\delta}^{\text {fo }}\right)$.

Theorem 3.1 (RDP and RSP Are Not Axiomatizable in $\mathcal{L}\left(\mathbf{B P A}_{\delta}^{\mathrm{fo}}\right)$ ). There does not exist a finitary first-order extension of $\mathrm{BPA}_{\delta}^{\text {fo }}$ of which all models satisfy RDP and RSP. 
Proof. It is sufficient to show that there does not exist a finitary first-order extension of $\mathrm{BPA}_{\delta}^{\text {fo }}$ of which all models satisfy RDP and there does not exist a finitary first-order extension of $\mathrm{BPA}_{\delta}^{\text {fo }}$ of which all models satisfy RSP.

First, we show that there does not exist a finitary first-order extension of $\mathrm{BPA}_{\delta}^{\text {fo }}$, say $\mathrm{BPA}_{\delta}^{\text {fo }} \cup H$, such that $\mathrm{BPA}_{\delta}^{\text {fo }} \cup H \models \mathrm{RDP}$. Suppose that $\mathrm{BPA}_{\delta}^{\text {fo }} \cup$ $H \models$ RDP. A contradiction is found as follows. By the Downward LöwenheimSkolem Theorem (see e.g. [24]), there exists a countable model of $\mathrm{BPA}_{\delta}^{\text {fo }} \cup H$. Take a countable model $\mathfrak{A} \models \mathrm{BPA}_{\delta}^{\text {fo }} \cup H$. Let $a$ and $b$ be different actions. Consider the guarded recursive specifications $E_{V}=\left\{X_{i}=a \cdot X_{i+1} \mid i \in V\right\} \cup$ $\left\{X_{i}=b \cdot X_{i+1} \mid i \notin V\right\}$ for $V \subseteq \mathbb{N}$. Then there exists a solution $p_{V}$ of $E_{V}$ for $X_{0}$ in $\mathfrak{A}$ for each $V \subseteq \mathbb{N}$. There exist uncountably many $V$ such that $V \subseteq \mathbb{N}$; and it is easily proved by induction on the smallest $i$ such that $i \in V \Leftrightarrow i \notin V^{\prime}$ that $V \neq V^{\prime}$ implies $p_{V} \neq p_{V^{\prime}}$. Hence, $\mathfrak{A}$ must be an uncountable model. However, $\mathfrak{A}$ is a countable model.

Next, we show that there does not exist a finitary first-order extension of $\mathrm{BPA}_{\delta}^{\text {fo }}$, say $\mathrm{BPA}_{\delta}^{\text {fo }} \cup H$, such that $\mathrm{BPA}_{\delta}^{\text {fo }} \cup H \models \mathrm{RSP}$. Suppose that $\mathrm{BPA}_{\delta}^{\text {fo }} \cup$ $H \models$ RSP. A contradiction is found as follows. Let $c_{0}, c_{1}, c_{2}, \ldots$ and $d_{0}, d_{1}, d_{2}, \ldots$ be different new constants; and let $a, a^{\prime}, a^{\prime \prime}$ be different actions. Consider the following sets of formulas:

$$
\begin{aligned}
H^{\prime}= & \left\{c_{0} \neq d_{0}\right\} \cup\left\{c_{i}=a \cdot c_{i+1} \mid i \geq 0\right\} \cup\left\{d_{i}=a \cdot d_{i+1} \mid i \geq 0\right\}, \\
H_{n}^{\prime}= & \left\{c_{0} \neq d_{0}\right\} \cup\left\{c_{i}=a \cdot c_{i+1} \mid 0 \leq i<n\right\} \cup\left\{d_{i}=a \cdot d_{i+1} \mid 0 \leq i<n\right\} \\
& \cup\left\{c_{n}=a^{\prime}, d_{n}=a^{\prime \prime}\right\}
\end{aligned}
$$

(for $n \geq 0$ ).

We can easily show that, for each $n \geq 0, H_{n}^{\prime}$ is satisfiable in an expansion of $\mathfrak{P}_{\aleph_{0}}$, a model of $\mathrm{BPA}_{\delta}^{\text {fo }}$ that will be introduced in Section 5 . Hence, for each $n \geq 0, H_{n}^{\prime}$ is consistent with $\mathrm{BPA}_{\delta}^{\text {fo }}$. Each finite $H^{\prime \prime} \subseteq H^{\prime}$ is consistent with $\mathrm{BPA}_{\delta}^{\text {fo }}$ because there is an $n \geq 0$ for which $H^{\prime \prime} \subseteq H_{n}^{\prime}$. From this, it follows by the Compactness Theorem (see e.g. [20]) that $H^{\prime}$ is consistent with $\mathrm{BPA}_{\delta}^{\text {fo }}$. Now consider an arbitrary model $\mathfrak{A}$ of $\mathrm{BPA}_{\delta}^{\text {fo }} \cup H \cup H^{\prime}$. Both $c_{0}^{\mathfrak{A}}$ and $d_{0}^{\mathfrak{A}}$ are solutions of the guarded recursive specification $E=\left\{X_{i}=a \cdot X_{i+1} \mid i \in \mathbb{N}\right\}$ for $X_{0}$. Hence, by RSP, it must be the case that $c_{0}^{\mathfrak{A}}=d_{0}^{\mathfrak{A}}$. However, $c_{0}^{\mathfrak{A}} \neq d_{0}^{\mathfrak{A}}$.

If we restrict ourselves to recursively enumerable theories, we can even give an instance of RDP that is not axiomatizable.

Theorem 3.2 (Instance of RDP Is Not Axiomatizable in $\mathcal{L}\left(\mathbf{B P A}_{\delta}^{\mathrm{fo}}\right)$ ). Let $T$ be a finitary first-order extension of $\mathrm{BPA}_{\delta}^{\text {fo }}$ that is recursively enumerable. Let $a, b$ be different actions. Let $V$ be a subset of $\mathbb{N}$ that is not recursively enumerable. Let $E_{V}$ be the guarded recursive specification $\left\{X_{i}=a \cdot X_{i+1} \mid i \in V\right\} \cup$ $\left\{X_{i}=b \cdot X_{i+1} \mid i \notin V\right\}$. Then $T \not \operatorname{RDP}^{E_{V}}$.

Proof. Let $\psi_{n}$, for each $n \geq 0$, be the following formula:

$$
\exists y \bullet \exists z_{0}, \ldots, z_{n} \bullet\left(x=z_{0} \cdot \ldots \cdot z_{n} \cdot y \wedge \bigwedge_{i \leq n, i \in V} z_{i}=a \wedge \bigwedge_{i \leq n, i \notin V} z_{i}=b\right) .
$$


Let $\Psi$ be the set of formulas $\left\{\psi_{n} \mid n \in \mathbb{N}\right\}$. It is easy to see that there does not exist a solution of $E_{V}$ in a model of $\mathrm{BPA}_{\delta}^{\text {fo }}$ iff that model omits $\Psi$. Moreover, by the Omitting Types Theorem (see e.g. [20]), there exists a model that omits $\Psi$ if $T$ or some consistent extension of $T$ locally omits $\Psi$. Thus, when we have shown that $T$ or some consistent extension of $T$ locally omits $\Psi$, we can immediately conclude that $T \not \mathrm{RDP}^{E_{V}}$ and we are done.

We prove that some consistent extension of $T$ locally omits $\Psi$ by constructing such an extension of $T$. Let $\phi_{0}, \phi_{1}, \phi_{2}, \ldots$ be an enumeration of all formulas of $\mathcal{L}\left(\mathrm{BPA}_{\delta}^{\text {fo }}\right)$ in which no variable other than $x$ has free occurrences. We start to construct a non-decreasing sequence $T^{0}, T^{1}, T^{2}, \ldots$ of consistent extensions of $T$ as follows:

$$
\begin{array}{rlrl}
T^{0} & =T, & \\
T^{2 k+1} & =T^{2 k} \cup\left\{\phi_{k}\right\} & & \text { if not } T^{2 k} \vdash \neg \phi_{k}, \\
T^{2 k+1} & =T^{2 k} \cup\left\{\neg \phi_{k}\right\} & & \text { otherwise }, \\
T^{2 k+2}=T^{2 k+1} & & \text { if } T^{2 k+1} \vdash \neg \exists x \bullet \phi_{k}(x), \\
T^{2 k+2} & =T^{2 k+1} \cup\left\{\exists x \bullet\left(\phi_{k}(x) \wedge \neg \psi_{n}(x)\right)\right\} & \text { otherwise }, \\
\text { for some } n \in \mathbb{N} \text { such that not } T^{2 k+1} \vdash \neg \exists x \bullet\left(\phi_{k}(x) \wedge \neg \psi_{n}(x)\right) .
\end{array}
$$

For all $k$, there exists an $n$ such that not $T^{2 k+1} \vdash \neg \exists x \bullet\left(\phi_{k}(x) \wedge \neg \psi_{n}(x)\right)$. This is easily proved by contradiction. If it was not the case, it would follow from the recursive enumerability of $T$ (and therefore also $T^{2 k+1}$ ) that $V$ is recursively enumerable. However, $V$ is not recursively enumerable.

For each $k \in \mathbb{N}, T^{k}$ is consistent by construction. Let $T^{\infty}=\bigcup_{k \in \mathbb{N}} T^{k}$. Then $T^{\infty}$ is also consistent by construction. Moreover, $T^{\infty}$ locally omits $\Psi$ by construction.

The bisimilarity axiom schema (BS) from Table 1 does not exclude all models that distinguish between processes that can be related by a bisimulation. It only excludes models that distinguish between processes that can be related by a first-order definable bisimulation. The second-order axiom B from Table 3 would exclude all such models. Axiom B is called the bisimilarity axiom. It is a secondorder axiom because of the existential quantification on $R$, which is a variable ranging over binary relations on processes instead of a variable ranging over processes.

The subprocess induction schema (RS) from Table 1 does not exclude all models in which there are processes that have more reachable processes than needed to satisfy axiom R1 and the instances of axiom schema R2. The secondorder axiom $\mathrm{R}$ from Table 3 would exclude all such models. Axiom $\mathrm{R}$ is called the subprocess induction axiom.

Let $\mathfrak{A}$ be a model of $\mathrm{BPA}_{\delta}^{\text {fo }}$, i.e. $\mathfrak{A} \models \mathrm{BPA}_{\delta}^{\text {fo }}$. Then $\mathfrak{A}$ is a bisimulation model if $\mathfrak{A} \models \mathrm{B}$; and $\mathfrak{A}$ is a model with standard reachability if $\mathfrak{A}=\mathrm{R}$. 
Table 3. Second-Order Axioms

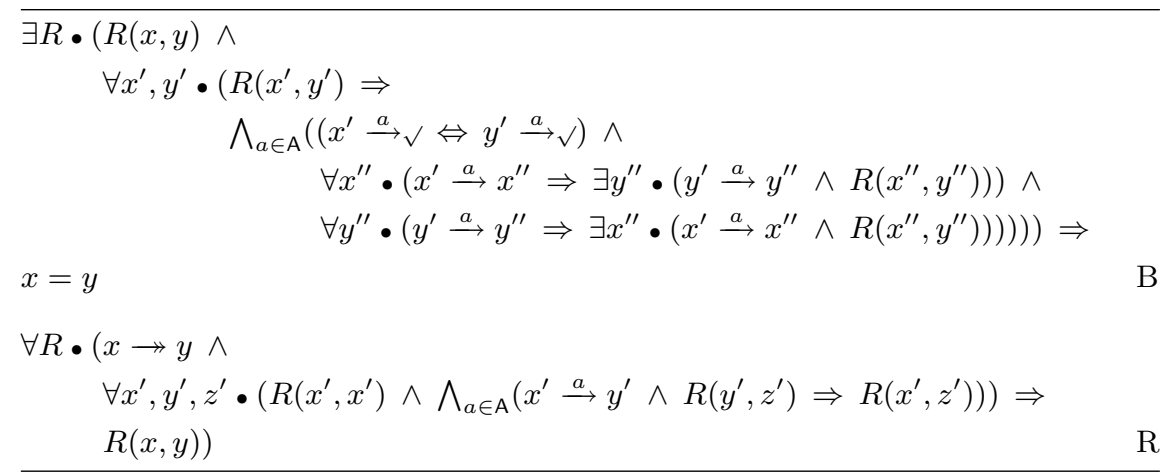

\section{Transition Systems and Bisimilarity}

In this section, we introduce transition systems and bisimilarity of transition systems. In Section 5, we will make use of transition systems and bisimilarity of transition systems to construct the main models of $\mathrm{BPA}_{\delta}^{\text {fo }}$.

A transition system $T$ consists of the following:

- a set $S$ of states;

- a set $\stackrel{a}{\longrightarrow} \subseteq S \times S$, for each $a \in \mathrm{A}$;

- a set $\stackrel{a}{\longrightarrow} \sqrt{ } \subseteq S$, for each $a \in \mathrm{A}$;

- an initial state $s^{0} \in S$.

If $\left(s, s^{\prime}\right) \in \stackrel{a}{\rightarrow}$ for some $a \in \mathrm{A}$, then we say that there is a transition from state $s$ to state $s^{\prime}$. We usually write $s \stackrel{a}{\rightarrow} s^{\prime}$ instead of $\left(s, s^{\prime}\right) \in \stackrel{a}{\rightarrow}$ and $s \stackrel{a}{\rightarrow} \sqrt{ }$ instead of $s \in \stackrel{a}{\rightarrow} \sqrt{ }$. Furthermore, we write $\rightarrow$ for the family of sets $(\stackrel{a}{\rightarrow})_{a \in \mathrm{A}}$ and $\rightarrow \sqrt{ }$ for the family of sets $(\stackrel{a}{\rightarrow} \sqrt{ })_{a \in \mathrm{A}}$.

The identity of the states of a transition system is not relevant to the behaviour represented by the transition system. We ignore the identity of the states of a transition system as follows.

Let $T_{1}=\left(S_{1}, \rightarrow_{1}, \rightarrow \sqrt{ }_{1}, s_{1}^{0}\right)$ and $T_{2}=\left(S_{2}, \rightarrow_{2}, \rightarrow \sqrt{ }_{2}, s_{2}^{0}\right)$ be transition systems. Then $T_{1}$ and $T_{2}$ are isomorphic if there exists a bijective function $b: S_{1} \rightarrow S_{2}$ such that

$$
\begin{aligned}
& -b\left(s_{1}^{0}\right)=s_{2}^{0} ; \\
& -s_{1} \stackrel{a}{\rightarrow} 1 s_{1}^{\prime} \text { iff } b\left(s_{1}\right) \stackrel{a}{\rightarrow} 2 b\left(s_{1}^{\prime}\right) ; \\
& -s \stackrel{a}{\rightarrow} \sqrt{ }_{1} \text { iff } b(s) \stackrel{a}{\rightarrow} \sqrt{ }_{2} .
\end{aligned}
$$

If $T_{1}$ and $T_{2}$ are isomorphic, then they differ only with respect to the identity of the states. Henceforth, we will always consider two transition systems the same if they are isomorphic, and write $T_{1}=T_{2}$ if $T_{1}$ and $T_{2}$ are isomorphic. Because of this, the disjointness requirement on the sets of states that occurs in the definitions of alternative composition and sequential composition on transition systems given in Section 5 does not cause any loss of generality. Moreover, it 
does not matter that an arbitrary fresh initial state is chosen in a few definitions from Section 5: up to isomorphism the result is independent of the particular choice.

A transition system may have states that are not reachable from its initial state by a number of transitions. Unreachable states, and the transitions between them, are not relevant to the behaviour represented by the transition system. We exclude transition systems with unreachable states as follows.

Let $T=\left(S, \rightarrow, \rightarrow \sqrt{ }, s^{0}\right)$ be a transition system. Then the reachability relation of $T$ is the smallest relation $\rightarrow \subseteq S \times S$ such that:

$$
\begin{aligned}
& -s \rightarrow s ; \\
& - \text { if } s \stackrel{a}{\rightarrow} s^{\prime} \text { and } s^{\prime} \rightarrow s^{\prime \prime} \text {, then } s \rightarrow s^{\prime \prime} .
\end{aligned}
$$

We write $\operatorname{RS}(T)$ for $\left\{s \in S \mid s^{0} \rightarrow s\right\}$. T is called a connected transition system if $S=\mathrm{RS}(T)$. Henceforth, we will only consider connected transition systems. However, this often calls for extraction of the connected part of a transition system that is composed of connected transition systems.

Let $T=\left(S, \rightarrow, \rightarrow \sqrt{ }, s^{0}\right)$ be a transition system that is not necessarily connected. Then the connected part of $T$, written $\Gamma(T)$, is defined as follows:

$$
\Gamma(T)=\left(S^{\prime}, \rightarrow^{\prime}, \rightarrow \sqrt{ }^{\prime}, s^{0}\right),
$$

where

$$
S^{\prime}=\mathrm{RS}(T)
$$

and for every $a \in \mathrm{A}$ :

$$
\begin{aligned}
& \stackrel{a}{\longrightarrow}=\stackrel{a}{\longrightarrow} \cap\left(S^{\prime} \times S^{\prime}\right), \\
& \stackrel{a}{\longrightarrow} \sqrt{ }^{\prime}=\stackrel{a}{\longrightarrow} \sqrt{ } \cap S^{\prime} .
\end{aligned}
$$

Let $\kappa$ be an infinite cardinal number. Then $\mathbb{T S}_{\kappa}$ is the set of all connected transition systems $T=\left(S, \rightarrow, \rightarrow \sqrt{ }, s^{0}\right)$ such that for all $s \in S$ the cardinality of the set $\left\{\left(a, s^{\prime}\right) \in \mathrm{A} \times S \mid s \stackrel{a}{\rightarrow} s^{\prime}\right\}$ is less than $\kappa$. $\mathbb{T S}_{\aleph_{0}}$ is the set of finitely branching connected transition systems. $\mathbb{T S}_{\aleph_{1}}$ is the set of countably branching connected transition systems.

Bisimilarity of transition systems from $\mathbb{T S}_{\kappa}$ is defined as follows.

Let $T_{1}=\left(S_{1}, \rightarrow_{1}, \rightarrow \sqrt{ }_{1}, s_{1}^{0}\right) \in \mathbb{T S}_{\kappa}$ and $T_{2}=\left(S_{2}, \rightarrow_{2}, \rightarrow \sqrt{ }_{2}, s_{2}^{0}\right) \in \mathbb{T} \mathbb{S}_{\kappa}(\kappa \geq$ $\left.\aleph_{0}\right)$. Then a bisimulation $B$ between $T_{1}$ and $T_{2}$ is a binary relation $B \subseteq S_{1} \times S_{2}$ such that $B\left(s_{1}^{0}, s_{2}^{0}\right)$ and for all $s_{1}, s_{2}$ such that $B\left(s_{1}, s_{2}\right)$ :

$-s_{1} \stackrel{a}{\rightarrow} \sqrt{ }_{1}$ iff $s_{2} \stackrel{a}{\longrightarrow} \sqrt{ }_{2}$;

- if $s_{1} \stackrel{a}{\longrightarrow} 1 s_{1}^{\prime}$, then there is a state $s_{2}^{\prime}$ such that $s_{2} \stackrel{a}{\longrightarrow}_{2} s_{2}^{\prime}$ and $B\left(s_{1}^{\prime}, s_{2}^{\prime}\right)$;

- if $s_{2} \stackrel{a}{\longrightarrow}_{2} s_{2}^{\prime}$, then there is a state $s_{1}^{\prime}$ such that $s_{1} \stackrel{a}{\longrightarrow}_{1} s_{1}^{\prime}$ and $B\left(s_{1}^{\prime}, s_{2}^{\prime}\right)$.

Two transition systems $T_{1}, T_{2} \in \mathbb{T} \mathbb{S}_{\kappa}$ are bisimilar, written $T_{1} \leftrightarrows T_{2}$, if there exists a bisimulation $B$ between $T_{1}$ and $T_{2}$. Let $B$ be a bisimulation between $T_{1}$ and $T_{2}$. Then we say that $B$ is a bisimulation witnessing $T_{1} \leftrightarrows T_{2}$. 
Note that $\leftrightarrows$ is an equivalence on $\mathbb{T S}_{\kappa}$. Let $T \in \mathbb{T S}_{\kappa}$. Then we write $[T]$ for

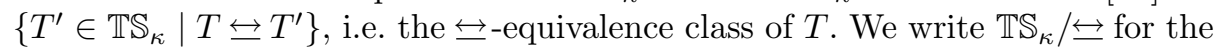
set of equivalence classes $\left\{[T] \mid T \in \mathbb{T S}_{\kappa}\right\}$.

It is easy to see that isomorphic transition systems are bisimilar. This justifies the choice to consider transition systems the same if they are isomorphic. It is easy to see that, if we would consider transition systems with unreachable states as well, each transition system would be bisimilar to its connected part. This justifies the choice to consider only connected transition systems.

In the construction of the main models of $\mathrm{BPA}_{\delta}^{\text {fo }}$ in Section 5, we also make use of subsystems of transition systems.

Let $T=\left(S, \rightarrow, \rightarrow \sqrt{ }, s^{0}\right) \in \mathbb{T S}_{\kappa}$ and $s \in S$. Then the subsystem of $T$ with initial state $s$, written $(T)_{s}$, is defined as follows:

$$
(T)_{s}=\Gamma(S, \rightarrow, \rightarrow \sqrt{ }, s) .
$$

\section{$5 \quad$ Full Bisimulation Models of $\mathrm{BPA}_{\delta}^{\text {fo }}$}

In this section, we introduce the full bisimulation models of $\mathrm{BPA}_{\delta}^{\text {fo }}$. They are models of which the domain consists of equivalence classes of transition systems modulo bisimilarity. The qualification "full" will be explained later on.

The models of $\mathrm{BPA}_{\delta}^{\text {fo }}$ are structures that consist of the following:

- a non-empty set $\mathcal{D}$, called the domain of the model;

- for each constant of $\mathrm{BPA}_{\delta}^{\text {fo }}$, an element of $\mathcal{D}$;

- for each $n$-ary operator of $\mathrm{BPA}_{\delta}^{\text {fo }}$, an $n$-ary operation on $\mathcal{D}$;

- for each $n$-ary predicate symbol of $\mathrm{BPA}_{\delta}^{\text {fo }}$, an $n$-ary relation on $\mathcal{D}$.

In the full bisimulation models of $\mathrm{BPA}_{\delta}^{\mathrm{fo}}$ that are introduced in this section, the domain is $\mathbb{T S}_{\kappa} / \leftrightarrows$ for some $\kappa \geq \aleph_{0}$. We obtain the models concerned by associating certain elements of $\mathbb{T S}_{\kappa} / \leftrightarrows$, certain operations on $\mathbb{T S} / \leftrightarrows$ and certain relations on $\mathbb{T S}_{\kappa} / \leftrightarrows$ with the constants, operators and predicate symbols of $\mathrm{BPA}_{\delta}^{\text {fo }}$. We begin by associating elements of $\mathbb{T S}_{\kappa}$ and operations on $\mathbb{T} \mathbb{S}_{\kappa}$ with the constants and operators, and a binary relation on $\mathbb{T S}_{\kappa}$ with the reachability predicate symbol. The result of this is subsequently lifted to $\mathbb{T} \mathbb{S}_{\kappa} / \leftrightarrows$.

We associate with each constant $c$ of $\mathrm{BPA}_{\delta}^{\text {fo }}$ an element $\widehat{c}$ of $\mathbb{T S}_{\kappa}$ and with each operator $f$ of $\mathrm{BPA}_{\delta}^{\text {fo }}$ an operation $\widehat{f}$ on $\mathbb{T} \mathbb{S}_{\kappa}$ as follows.

$$
\begin{gathered}
-\quad \widehat{\delta}=\left(\left\{s^{0}\right\}, \emptyset, \emptyset, s^{0}\right) . \\
-\quad \widehat{a}=\left(\left\{s^{0}\right\}, \emptyset, \rightarrow \sqrt{ }, s^{0}\right), \\
\text { where } \\
\stackrel{a}{\rightarrow} \sqrt{ }=\left\{s^{0}\right\},
\end{gathered}
$$

and for every $a^{\prime} \in \mathrm{A}$ such that $a^{\prime} \neq a$ :

$$
\stackrel{a^{\prime}}{\longrightarrow} \sqrt{ }=\emptyset \text {. }
$$


- Let $T_{i}=\left(S_{i}, \rightarrow_{i}, \rightarrow \vee_{i}, s_{i}^{0}\right) \in \mathbb{T S}_{\kappa}$ for $i=1,2, S_{1} \cap S_{2}=\emptyset$, and $s^{0} \notin S_{1} \cup S_{2}$.

Then

$$
T_{1} \widehat{+} T_{2}=\Gamma\left(S, \rightarrow, \rightarrow \sqrt{ }, s^{0}\right),
$$

where

$$
S=\left\{s^{0}\right\} \cup S_{1} \cup S_{2},
$$

and for every $a \in \mathrm{A}$ :

$$
\begin{aligned}
& \stackrel{a}{\rightarrow}=\left\{\left(s^{0}, s\right) \mid s_{1}^{0} \stackrel{a}{\rightarrow}_{1} s \vee s_{2}^{0} \stackrel{a}{\rightarrow}_{2} s\right\} \cup \stackrel{a}{\rightarrow} \cup \cup \stackrel{a}{\rightarrow}_{2}, \\
& \stackrel{a}{\rightarrow} \sqrt{ }=\left\{s^{0} \mid s_{1}^{0} \stackrel{a}{\rightarrow} \sqrt{ }_{1} \vee s_{2}^{0} \stackrel{a}{\rightarrow} \sqrt{ }_{2}\right\} \cup \stackrel{a}{\rightarrow} \sqrt{ }_{1} \cup \stackrel{a}{\rightarrow} \sqrt{ }_{2} .
\end{aligned}
$$

- Let $T_{i}=\left(S_{i}, \rightarrow_{i}, \rightarrow_{\sqrt{ }}, s_{i}^{0}\right) \in \mathbb{T S}_{\kappa}$ for $i=1,2$, and $S_{1} \cap S_{2}=\emptyset$. Then

$$
T_{1} \widehat{\cdot} T_{2}=\Gamma\left(S, \rightarrow, \rightarrow \sqrt{ }, s_{1}^{0}\right),
$$

where

$$
S=S_{1} \cup S_{2},
$$

and for every $a \in \mathrm{A}$ :

$$
\begin{aligned}
& \stackrel{a}{\rightarrow}=\stackrel{a}{\rightarrow}_{1} \cup\left\{\left(s, s_{2}^{0}\right) \mid s \stackrel{a}{\rightarrow} \sqrt{ }_{1}\right\} \cup \stackrel{a}{\rightarrow}_{2}, \\
& \stackrel{a}{\rightarrow} \sqrt{ }=\sqrt{ }_{2} .
\end{aligned}
$$

We associate with the reachability predicate symbol $\rightarrow$ a relation $\widehat{\rightarrow}$ on $\mathbb{T} \mathbb{S}_{\kappa}$ as follows.

$$
\begin{gathered}
\text { - Let } T_{i}=\left(S_{i}, \rightarrow_{i}, \rightarrow{ } i, s_{i}^{0}\right) \in \mathbb{T} \mathbb{S}_{\kappa} \text { for } i=1,2 . \text { Then } \\
T_{1} \widehat{\rightarrow} T_{2} \text { iff } \exists s \in S_{1} \bullet\left(T_{1}\right)_{s}=T_{2} .
\end{gathered}
$$

Recall that the disjointness requirement on the sets of states and the choice of an arbitrary fresh initial state in the definitions given above do not really matter because isomorphic transition systems are considered the same. In the definition of alternative composition on $\mathbb{T S}_{\kappa}$, the connected part of a transition system is extracted because the initial states of the transition systems $T_{1}$ and $T_{2}$ may be unreachable from the new initial state. The new initial state is introduced because, in $T_{1}$ and/or $T_{2}$, there may exist a transition back to the initial state. In the definition of sequential composition on $\mathbb{T S}_{\kappa}$, the connected part of a transition system is extracted because the initial state of the transition system $T_{2}$ may be unreachable from the initial state of the transition system $T_{1}$ - due to absence of termination in $T_{1}$.

We do not associate relations on $\mathbb{T S} \mathbb{S}_{\kappa}$ with the summand inclusion, action termination and action step predicate symbols. They have defining axioms, which explicitly define them in terms of the other nonlogical symbols of $\mathrm{BPA}_{\delta}^{\text {fo }}$. Therefore, it is known how to obtain the relations on $\mathbb{T S}_{\kappa} / \leftrightarrows$ to be associated with these predicate symbols from the elements of $\mathbb{T S}_{\kappa} / \leftrightarrows$, operations on $\mathbb{T} \mathbb{S}_{\kappa} / \leftrightarrows$ and relations on $\mathbb{T S}_{\kappa} / \leftrightarrows$ to be associated with the other nonlogical symbols of $\mathrm{BPA}_{\delta}^{\text {fo }}$.

We can easily show that bisimilarity is a congruence with respect to alternative composition and sequential composition. 
Proposition 5.1 (Congruence). For all $T_{1}, T_{2}, T_{1}^{\prime}, T_{2}^{\prime} \in \mathbb{T S}_{\kappa}\left(\kappa \geq \aleph_{0}\right), T_{1} \leftrightarrows$ $T_{1}^{\prime}$ and $T_{2} \leftrightarrows T_{2}^{\prime}$ imply $T_{1} \widehat{+} T_{2} \leftrightarrows T_{1}^{\prime} \hat{+} T_{2}^{\prime}$ and $T_{1} \widehat{\cdot} T_{2} \leftrightarrows T_{1}^{\prime} \widehat{\cdot} T_{2}^{\prime}$.

Proof. Let $R_{1}$ and $R_{2}$ be bisimulations witnessing $T_{1} \leftrightarrow T_{1}^{\prime}$ and $T_{2} \leftrightarrows T_{2}^{\prime}$, respectively. Then we construct relations $R_{\widehat{+}}$ and $R$ ? as follows:

- $R_{\widehat{+}}=\left(\left\{\left(s^{0}, s^{0 \prime}\right)\right\} \cup R_{1} \cup R_{2}\right) \cap\left(S \times S^{\prime}\right)$, where $S$ and $S^{\prime}$ are the sets of states of $T_{1} \widehat{+} T_{2}$ and $T_{1}^{\prime} \hat{+} T_{2}^{\prime}$, respectively, and $s^{0}$ and $s^{0 \prime}$ are the initial states of $T_{1} \widehat{+} T_{2}$ and $T_{1}^{\prime} \widehat{+} T_{2}^{\prime}$, respectively;

- $R_{\mathrm{r}}=\left(R_{1} \cup R_{2}\right) \cap\left(S \times S^{\prime}\right)$, where $S$ and $S^{\prime}$ are the sets of states of $T_{1} \hat{\cdot} T_{2}$ and $T_{1}^{\prime} \widehat{\cdot} T_{2}^{\prime}$, respectively.

Given the definitions of alternative composition and sequential composition, it

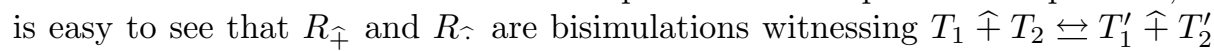
and $T_{1} \widehat{\cdot} T_{2} \leftrightarrows T_{1}^{\prime} \widehat{\cdot} T_{2}^{\prime}$, respectively. ing: ${ }^{1}$

The full bisimulation models $\mathfrak{P}_{\kappa}$, one for each $\kappa \geq \aleph_{0}$, consist of the follow-

- a set $\mathcal{P}$, called the domain of $\mathfrak{P}_{\kappa}$;

- for each constant $c$ of $\mathrm{BPA}_{\delta}^{\mathrm{fo}}$, an element $\widetilde{c}$ of $\mathcal{P}$;

- for each $n$-ary operator $f$ of $\mathrm{BPA}_{\delta}^{\text {fo }}$, an $n$-ary operation $\widetilde{f}$ on $\mathcal{P}$;

- for each $n$-ary predicate symbol $R$ of $\mathrm{BPA}_{\delta}^{\text {fo }}$, a $n$-ary relation $\widetilde{R}$ on $\mathcal{P}$;

where those ingredients are defined as follows:

$$
\begin{aligned}
& \mathcal{P} \quad=\mathbb{T S}_{\kappa} / \leftrightarrows, \\
& \widetilde{\delta} \quad=[\widehat{\delta}], \\
& \widetilde{a}=[\widehat{a}], \\
& {\left[T_{1}\right] \widetilde{+}\left[T_{2}\right]=\left[T_{1} \widehat{+} T_{2}\right],} \\
& {\left[T_{1}\right] \sim\left[T_{2}\right]=\left[T_{1} \widehat{\cdot} T_{2}\right],} \\
& {\left[T_{1}\right] \widetilde{\subseteq}\left[T_{2}\right] \text { iff }\left[T_{1}\right] \widetilde{+}\left[T_{2}\right]=\left[T_{2}\right],} \\
& {\left[T_{1}\right] \stackrel{\widetilde{a}}{\rightarrow} \sqrt{ } \quad \text { iff } \widetilde{a} \widetilde{\Xi}\left[T_{1}\right],} \\
& {\left[T_{1}\right] \stackrel{\widetilde{a}}{\rightarrow}\left[T_{2}\right] \text { iff } \widetilde{a} \widetilde{r}\left[T_{2}\right] \widetilde{\subseteq}\left[T_{1}\right],}
\end{aligned}
$$

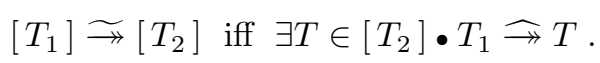

Alternative composition and sequential composition on $\mathbb{T S}_{\kappa} / \leftrightarrows$ are well-defined

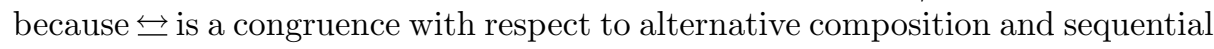
composition on $\mathbb{T \mathbb { S } _ { \kappa }}$. Reachability on $\mathbb{T S}_{\kappa} / \leftrightarrows$ is well-defined because $\leftrightarrows$ preserves

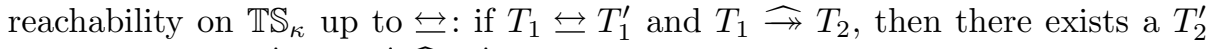
such that $T_{2} \leftrightarrows T_{2}^{\prime}$ and $T_{1}^{\prime} \widehat{\rightarrow} T_{2}^{\prime}$.

The structures $\mathfrak{P}_{\kappa}$ are models of $\mathrm{BPA}_{\delta}^{\text {fo }}$.

\footnotetext{
${ }^{1} \mathfrak{P}$ is the Gothic capital P.
} 
Theorem 5.1 (Soundness of $\mathbf{B P A}_{\delta}^{\text {fo }}$ ). For all $\kappa \geq \aleph_{0}$, we have $\mathfrak{P}_{\kappa} \models \mathrm{BPA}_{\delta}^{\text {fo }}$.

Proof. The soundness of all axioms, except RDPf and RSPf, follows easily from the definitions of the ingredients of $\mathfrak{P}_{\kappa}$. The soundness of RDPf and RSPf follows immediately from Theorem 5.2 (see below), which states the soundness of RDP and RSP.

All finite and countably infinite guarded recursive specifications have a unique solution in the full bisimulation models.

Theorem 5.2 (Soundness of RDP and RSP). For all $\kappa \geq \aleph_{0}$, we have $\mathfrak{P}_{\kappa} \models \mathrm{RDP}$ and $\mathfrak{P}_{\kappa} \models \mathrm{RSP}$

Proof. This is essentially the proof of soundness of RDP and RSP in the graph models of $\mathrm{ACP}_{\tau}$ given in [5] adapted to the case without silent steps.

Moreover, $\mathrm{B}$ and $\mathrm{R}$ are valid in the full bisimulation models.

Theorem 5.3 (Soundness of $\mathbf{B}$ and $\mathbf{R}$ ). For all $\kappa \geq \aleph_{0}$, we have $\mathfrak{P}_{\kappa}=\mathrm{B}$ and $\mathfrak{P}_{\kappa} \models \mathrm{R}$.

Proof. The soundness of B follows easily from the definitions of $\underset{a}{\rightarrow} \sqrt{ }$ and $\stackrel{\widetilde{a}}{\rightarrow}$, the definition of bisimilarity of transition systems and Proposition 6.1. The soundness of $\mathrm{R}$ follows easily from the definitions of $\underset{\vec{a}}{\rightarrow}$ and $\widetilde{\rightarrow}$, the definition of the reachability relation of a transition system and Corollary 10.1. ${ }^{2}$

As to be expected, the full bisimulation models are related by isomorphic embeddings.

Theorem 5.4 (Isomorphic Embedding). Let $\aleph_{0} \leq \kappa<\kappa^{\prime}$. Then $\mathfrak{P}_{\kappa}$ is isomorphically embedded in $\mathfrak{P}_{\kappa^{\prime}}$.

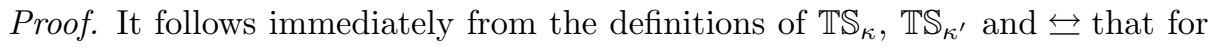
each $p \in \mathbb{T S}_{\kappa} / \leftrightarrows$, there exists a unique $p^{\prime} \in \mathbb{T S}_{\kappa^{\prime}} / \leftrightarrows$ such that $p \subseteq p^{\prime}$. Now

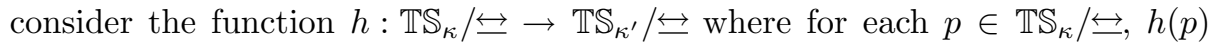
is the unique $p^{\prime} \in \mathbb{T S}_{\kappa^{\prime}} / \leftrightarrows$ such that $p \subseteq p^{\prime}$. It follows immediately from the definition of $h$ that $h$ is injective. Moreover, it follows easily from the definitions of the operations and relations on $\mathbb{T} \mathbb{S}_{\kappa} / \leftrightarrows$ and $\mathbb{T S}_{\kappa^{\prime}} / \leftrightarrows$ that $h$ is a homomorphism from $\mathfrak{P}_{\kappa}$ to $\mathfrak{P}_{\kappa^{\prime}}$.

In Section 6, we will show that every bisimulation model with standard reachability, i.e. every model that additionally satisfies the second-order axioms B and R, is isomorphically embedded in the models $\mathfrak{P}_{\kappa}$ from some $\kappa \geq \aleph_{0}$. This explains why the models $\mathfrak{P}_{\kappa}$ are called full bisimulation models: within the bound on the branching degree set by $\kappa, \mathfrak{P}_{\kappa}$ is full.

The question whether all full bisimulation models are elementary equivalent must be answered in the negative. ${ }^{2}$ Proposition 6.1 and Corollary 10.1 are not proved here, because they need definitions
of auxiliary notions which are better in place in Sections 6 and 10, respectively. 
Theorem 5.5 (No Elementary Equivalence). We have $\mathfrak{P}_{\aleph_{0}} \not \equiv \mathfrak{P}_{2^{\aleph_{0}}}, \mathfrak{P}_{\aleph_{0}} \not \equiv$ $\mathfrak{P}_{2^{2^{\aleph_{0}}}}$ and $\mathfrak{P}_{2^{\aleph_{0}}} \not \equiv \mathfrak{P}_{2^{2^{\aleph_{0}}}}$.

Proof. $\mathfrak{P}_{\aleph_{0}} \not \equiv \mathfrak{P}_{2^{\aleph_{0}}}$ and $\mathfrak{P}_{\aleph_{0}} \not \equiv \mathfrak{P}_{2^{2^{\aleph_{0}}}}$ are proved as follows. Let $a$ be an action. Let $\phi$ be the following formula of $\mathcal{L}\left(\mathrm{BPA}_{\delta}^{\text {fo }}\right)$ :

$$
\exists x \bullet(x \stackrel{a}{\longrightarrow} \delta \wedge \forall y \bullet(x \stackrel{a}{\longrightarrow} y \Rightarrow \exists z \bullet(z \neq y \wedge x \stackrel{a}{\longrightarrow} z \wedge z \stackrel{a}{\longrightarrow} y))) .
$$

Clearly, $\mathfrak{P}_{\aleph_{0}} \not \models \phi$, but $\mathfrak{P}_{2^{\aleph_{0}}} \models \phi$ and $\mathfrak{P}_{2^{2^{\aleph_{0}}}} \models \phi$.

$\mathfrak{P}_{2^{\aleph_{0}}} \not \equiv \mathfrak{P}_{2^{2^{\aleph_{0}}}}$ is proved as follows. Let $a, a^{\prime}, b, b^{\prime}$ be different actions. Let $\phi$ be the following formula of $\mathcal{L}\left(\mathrm{BPA}_{\delta}^{\text {fo }}\right)$ :

$$
\begin{aligned}
& \exists ! y \bullet(x \stackrel{a}{\rightarrow} y \wedge \\
& \left.\quad \forall y, z \bullet\left(x \rightarrow y \wedge y \stackrel{a}{\rightarrow} z \Rightarrow \exists ! u \bullet z \stackrel{a}{\rightarrow} u \wedge \neg\left(z \stackrel{a^{\prime}}{\longrightarrow} \sqrt{ } \Leftrightarrow z \stackrel{b^{\prime}}{\longrightarrow} \sqrt{ }\right)\right)\right) .
\end{aligned}
$$

For all $\kappa \geq \aleph_{0}$, there exist $2^{\aleph_{0}}$ different $x$ in the domain of $\mathfrak{P}_{\kappa}$ for which $\phi(x)$. Let $\psi$ be the following formula of $\mathcal{L}\left(\mathrm{BPA}_{\delta}^{\text {fo }}\right)$ :

$$
\exists w \bullet(\forall x \bullet(\phi(x) \Rightarrow w \stackrel{b}{\rightarrow} x)) .
$$

Clearly, $\mathfrak{P}_{2^{\aleph_{0}}} \not \models \psi$ and $\mathfrak{P}_{2^{2^{\aleph_{0}}}} \models \psi$.

We conjecture that, more general, $\mathfrak{P}_{\kappa} \not \equiv \mathfrak{P}_{\kappa^{\prime}}$ if $\kappa \neq \kappa^{\prime}$.

\section{External Bisimilarity}

Each model of $\mathrm{BPA}_{\delta}^{\text {fo }}$ induces a transition system for each element of its domain.

Let $\mathfrak{A}$ be a model of $\mathrm{BPA}_{\delta}^{\text {fo }}$ with domain $P$, a binary relation $\stackrel{a}{\rightarrow}$ on $P$ for each predicate symbol $\stackrel{a}{\rightarrow}$, and a unary relation $\stackrel{a}{\rightarrow}{ }^{\prime}$ on $P$ for each predicate symbol $\stackrel{a}{\rightarrow} \sqrt{ }$. Moreover, let $p \in P$. Then the transition system of $p$ induced by $\mathfrak{A}$, written $\operatorname{TS}(\mathfrak{A}, p)$, is defined as follows:

$$
\operatorname{TS}(\mathfrak{A}, p)=\Gamma\left(P, \rightarrow^{\prime}, \rightarrow \sqrt{ }^{\prime}, p\right) .
$$

In each of the full bisimulation models, every element of the domain is an equivalence class of transition systems. The transition system of an element induced by the model is (up to isomorphism) a representative of that element.

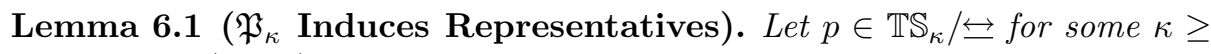
$\aleph_{0}$. Then $\operatorname{TS}\left(\mathfrak{P}_{\kappa}, p\right) \in p$.

Proof. Let $\operatorname{TS}\left(\mathfrak{P}_{\kappa}, p\right)=\left(P, \rightarrow^{\prime}, \rightarrow \vee^{\prime}, p\right)$. Take an arbitrary transition system $T=\left(S, \rightarrow^{\prime \prime}, \rightarrow \mathcal{J}^{\prime \prime}, s^{0}\right) \in \mathbb{T S}_{\kappa}$ such that $[T]=p$. Consider the relation $B \subseteq P \times S$ defined as follows:

$$
B=\left\{\left(\left[(T)_{s}\right], s\right) \mid s \in S\right\} .
$$

It is easy to see that $B$ is a bisimulation between $\operatorname{TS}\left(\mathfrak{P}_{\kappa}, p\right)$ and $T$. Hence, $\mathrm{TS}\left(\mathfrak{P}_{\kappa}, p\right) \in[T]=p$. 
Let $\mathfrak{A}$ be a model of $\mathrm{BPA}_{\delta}^{\text {fo }}$ with domain $P$. Then bisimilarity on $P$ is defined as follows:

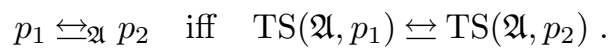

Bisimilarity on the domain of a model of $\mathrm{BPA}_{\delta}^{\text {fo }}$ as defined above is called $e x$ ternal bisimilarity. In each of the full bisimulation models, external bisimilarity coincides with identity.

Proposition 6.1 (External Bisimilarity Is Identity in $\mathfrak{P}_{\kappa}$ ). Let $p_{1}, p_{2} \in$

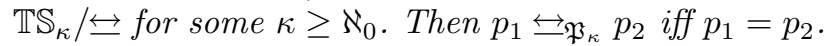

Proof. Follows immediately from Lemma 6.1.

There does not exist a consistent extension of $\mathrm{BPA}_{\delta}^{\text {fo }}$ with first-order axioms that has only models in which external bisimilarity coincides with identity.

Theorem 6.1 (Undefinability of External Bisimilarity). External bisimilarity is not first-order definable in any consistent extension of $\mathrm{BPA}_{\delta}^{\text {fo }}$.

Proof. From Proposition 6.1, we know that it is sufficient to show that each firstorder consistent extension of $\mathrm{BPA}_{\delta}^{\text {fo }}$ has a model in which external bisimilarity is not identity.

Suppose that each first-order consistent extension of $\mathrm{BPA}_{\delta}^{\text {fo }}$ has only models in which external bisimilarity is identity. A contradiction is found as follows. Let $c_{0}, c_{1}, c_{2}, \ldots$ and $d_{0}, d_{1}, d_{2}, \ldots$ be different new constants; and let $a, a^{\prime}, a^{\prime \prime}$ be different actions. Consider the following sets of formulas:

$$
\begin{aligned}
& H=\left\{c_{0} \neq d_{0}\right\} \cup\left\{c_{i}=a \cdot c_{i+1} \mid i \geq 0\right\} \cup\left\{d_{i}=a \cdot d_{i+1} \mid i \geq 0\right\}, \\
& H_{n}=\left\{c_{0} \neq d_{0}\right\} \cup\left\{c_{i}=a \cdot c_{i+1} \mid 0 \leq i<n\right\} \cup\left\{d_{i}=a \cdot d_{i+1} \mid 0 \leq i<n\right\} \\
& \cup\left\{c_{n}=a^{\prime}, d_{n}=a^{\prime \prime}\right\} \\
&\text { (for } n \geq 0) .
\end{aligned}
$$

For each $n \geq 0, H_{n}$ is satisfiable in an expansion of $\mathfrak{P}_{\aleph_{0}}$. Hence, for each $n \geq 0$, $H_{n}$ is consistent with $\mathrm{BPA}_{\delta}^{\text {fo }}$. Each finite $H^{\prime} \subseteq H$ is consistent with $\mathrm{BPA}_{\delta}^{\text {fo }}$ because there is an $n \geq 0$ for which $H^{\prime} \subseteq H_{n}$. From this, it follows by the Compactness Theorem that $H$ is consistent with $\mathrm{BPA}_{\delta}^{\text {fo }}$. Now consider an arbitrary model $\mathfrak{A}$ of $\mathrm{BPA}_{\delta}^{\text {fo }} \cup H$. Because $\operatorname{TS}\left(\mathfrak{A}, c_{0}^{\mathfrak{A}}\right)$ and $\mathrm{TS}\left(\mathfrak{A}, d_{0}^{\mathfrak{A}}\right)$ are isomorphic transition systems, $c_{0}^{\mathfrak{A}} \leftrightarrows_{\mathfrak{A}} d_{0}^{\mathfrak{A}}$. However, $c_{0}^{\mathfrak{A}} \neq d_{0}^{\mathfrak{A}}$.

We can summarize the state of affairs as follows. It is obvious that equality derivable from $\mathrm{BPA}_{\delta}^{\text {fo }}$ implies external bisimilarity in each model of $\mathrm{BPA}_{\delta}^{\text {fo }}$. In the full bisimulation models, external bisimilarity coincides with identity. However, there also exist models of which the domain contains pairs of different elements that are externally bisimilar. Moreover, those models cannot be excluded by extending $\mathrm{BPA}_{\delta}^{\text {fo }}$ with first-order axioms.

The above-mentioned discrepancy can for the greater part be eliminated in second-order logic, as indicated below by Theorem 6.2 . This theorem states that each bisimulation model with standard reachability is isomorphic to a substructure of one of the full bisimulation models. 
Theorem 6.2 (Isomorphic Embedding). Let $\mathfrak{A}$ be a model of $\mathrm{BPA}_{\delta}^{\text {fo }}$ such that $\mathfrak{A}=\mathrm{R}$. Then $\mathfrak{A} \models \mathrm{B}$ iff $\mathfrak{A}$ is isomorphically embedded in $\mathfrak{P}_{\kappa}$ for some $\kappa \geq \aleph_{0}$.

Proof. The implication from left to right is proved as follows. Let $P$ be the domain of $\mathfrak{A}, \kappa^{\prime}$ be the cardinality of $P$, and $\kappa>\kappa^{\prime}$. It follows immediately from the definitions of $\mathrm{TS}$ and $\mathbb{T S}_{\kappa}$ that for each $p \in P$, TS $(\mathfrak{A}, p) \in \mathbb{T S}_{\kappa}$. Now consider the function $h: P \rightarrow \mathbb{T S}_{\kappa} / \leftrightarrows$ such that for each $p \in P, h(p)=[\mathrm{TS}(\mathfrak{A}, p)]$. Because $\mathfrak{A} \models \mathrm{B}$, it follows immediately that $h$ is injective. Because the implications from right to left are derivable, the occurrence of " $\Rightarrow$ " in axioms SI7-SI9 (Table 1) can be replaced by " $\Leftrightarrow$ ". It follows easily from these equivalences and the definitions of alternative composition and sequential composition on $\mathbb{T} \mathbb{S}_{\kappa} / \leftrightarrows$ (Section 5 ) that $h$ is a homomorphism with respect to these operations. From this, it follows immediately by axioms SI1, TR1 and TR2 that $h$ is also a homomorphism with respect to the summand inclusion, action termination and action step relations. Because $\mathfrak{A} \models \mathrm{R}$, it follows immediately that $h$ is a homomorphism with respect to the reachability relation. The implication from right to left is trivial.

\section{Observational Equivalence}

In this section, we have a closer look at observational equivalence as defined in [23]. This equivalence on the domain of models of $\mathrm{BPA}_{\delta}^{\text {fo }}$ is closely related to external bisimilarity. Observational equivalence is defined in the following way.

Let $\mathfrak{A}$ be a model of $\mathrm{BPA}_{\delta}^{\text {fo }}$ with domain $P$, a binary relation $\stackrel{a}{\rightarrow}$ on $P$ for each predicate symbol $\stackrel{a}{\rightarrow}$, and a unary relation $\stackrel{a}{\rightarrow} \sqrt{ }^{\prime}$ on $P$ for each predicate symbol $\stackrel{a}{\rightarrow} \sqrt{ }$. Then equivalences $\approx_{n} \subseteq P \times P$ for each $n \geq 0$ are defined as follows:

$-p_{1} \approx_{0} p_{2}$ for all $p_{1}, p_{2} \in P$

$-p_{1} \approx_{n+1} p_{2}$ if

- $p_{1} \stackrel{a}{\rightarrow} \sqrt{ }^{\prime}$ iff $p_{2} \stackrel{a}{\rightarrow} \sqrt{ }^{\prime}$

- if $p_{1} \stackrel{a}{\rightarrow} p_{1}^{\prime}$, then there is a $p_{2}^{\prime} \in P$ such that $p_{2} \stackrel{a}{\rightarrow} p_{2}^{\prime}$ and $p_{1}^{\prime} \approx_{n} p_{2}^{\prime}$;

- if $p_{2} \stackrel{a}{\rightarrow} p_{2}^{\prime}$, then there is a $p_{1}^{\prime} \in P$ such that $p_{1} \stackrel{a}{\rightarrow} p_{1}^{\prime}$ and $p_{1}^{\prime} \approx_{n} p_{2}^{\prime}$.

Now, $p_{1}$ and $p_{2}$ are observationally equivalent in $\mathfrak{A}$, written $p_{1} \approx_{\mathfrak{A}} p_{2}$, if $p_{1} \approx_{n} p_{2}$ for all $n \geq 0$.

If all transition systems that can be extracted from a model are finitely branching, then observational equivalence and external bisimilarity coincide.

Theorem 7.1 (Observational Equivalence vs External Bisimilarity).

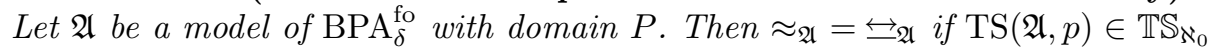
for all $p \in P$.

Proof. The proof is analogous to the proof of the corresponding property for process graphs given in [13].

An interesting extension of $\mathrm{BPA}_{\delta}^{\text {fo }}$ is obtained as follows. We add to the nonlogical symbols of $\mathrm{BPA}_{\delta}^{\text {fo }}$, for each $n \geq 0$, a binary observational equivalence 
Table 4. Approximation Induction Principle

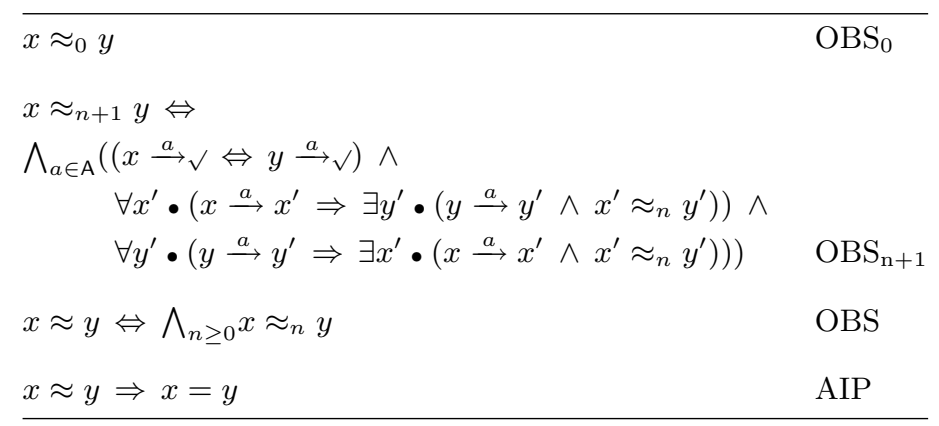

up to depth $n$ predicate symbol $\approx_{n}$ and a binary observational equivalence predicate symbol $\approx$. Moreover, we add the axioms given in Table 4 to the axioms of $\mathrm{BPA}_{\delta}^{\text {fo }}$. Axiom $\mathrm{OBS}_{0}$ is the defining axiom of the observational equivalence up to depth 0 predicate; and $\mathrm{OBS}_{\mathrm{n}+1}$ is an axiom schema whose instances are the defining axioms of the observational equivalence up to depth $n+1$ predicates. Axiom OBS is the defining axiom of the observational equivalence predicate. Axiom AIP is called the approximation induction principle. We write $\mathfrak{P} \approx(\kappa \geq$ $\aleph_{0}$ ) for the unique definitional expansion of $\mathfrak{P}_{\kappa}$ determined by the definitional extension of $\mathrm{BPA}_{\delta}^{\text {fo }}$ with axioms $\mathrm{OBS}_{0}, \mathrm{OBS}_{1}, \ldots$ and OBS. AIP is valid in $\mathfrak{P}_{\aleph_{0}}$, but not in $\mathfrak{P} \approx$ with $\kappa \geq \aleph_{1}$.

Theorem 7.2 (Soundness of AIP). We have $\mathfrak{P} \widetilde{\tilde{\kappa}} \models$ AIP iff $\kappa=\aleph_{0}$.

Proof. It follows immediately from Proposition 6.1 and Theorem 7.1 that $\mathfrak{P} \approx \bar{\kappa} \models$ AIP if $\kappa=\aleph_{0}$. For $\kappa>\aleph_{0}$, we have the following counterexample. Fix an $a \in \mathrm{A}$. Consider the transition systems $T_{1}=\left(S_{1}, \rightarrow_{1}, \emptyset, 0\right)$ and $T_{2}=\left(S_{2}, \rightarrow_{2}, \emptyset, 0\right)$ where

$$
\begin{aligned}
& S_{1} \quad=\{0\} \cup\{(i, j) \mid i, j \in \mathbb{N}, i \geq j \geq 1\}, \\
& \stackrel{a}{\longrightarrow}_{1}=\{(0,(i, 1)) \mid i \in \mathbb{N}, i \geq 1\} \cup\{((i, j),(i, j+1)) \mid i, j \in \mathbb{N}, i>j \geq 1\}, \\
& \stackrel{a^{\prime}}{\longrightarrow}=\emptyset \text { for every } a^{\prime} \in \mathrm{A} \text { such that } a^{\prime} \neq a,
\end{aligned}
$$

and

$$
\begin{aligned}
& S_{2}=S_{1} \cup \mathbb{N}, \\
& \stackrel{a}{\longrightarrow} 2=\stackrel{a}{\rightarrow} \cup\{(i, i+1) \mid i \in \mathbb{N}\}, \\
& \stackrel{a^{\prime}}{\longrightarrow} 2=\emptyset \text { for every } a^{\prime} \in \text { A such that } a^{\prime} \neq a .
\end{aligned}
$$

Clearly, $T_{1}, T_{2} \notin \mathbb{T} \mathbb{S}_{\aleph_{0}}$. Because $T_{1}$ has no infinite branch and $T_{2}$ has an infinite branch, $T_{1} \sharp_{\mathfrak{P}} \widetilde{\kappa} T_{2}$. However, $T_{1} \approx_{\mathfrak{P} \approx} T_{2}$.

All models that satisfy AIP, satisfy B as well.

Proposition 7.1 (AIP Implies B). We have AIP $\models$ B. 
Table 5. Axiom Schema for the Constants $\langle X \mid E\rangle$

$$
\overline{\bigwedge_{1 \leq i \leq n}\left\langle X_{i} \mid E\right\rangle=t_{i}\left(\left\langle X_{1} \mid E\right\rangle, \ldots,\left\langle X_{n} \mid E\right\rangle\right) \quad \text { if } E=\left\{X_{i}=t_{i} \mid 1 \leq i \leq n\right\} \quad \text { RDPc }}
$$

Proof. Take a model $\mathfrak{A}$ of $\mathrm{BPA}_{\delta}^{\text {fo }}$ with domain $P$. Let $p, p^{\prime} \in P$. It is easily proved

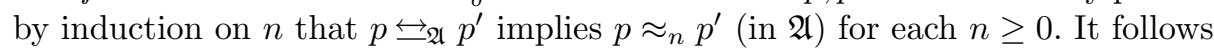
immediately that, if AIP is satisfied, B is satisfied as well.

In $[15,16]$, more can be found on bisimulation models in which AIP is valid. In those papers, only bisimulation models of PA, i.e. ACP without communication (see also Section 13), are considered.

Note that the defining axiom of observational equivalence is a formula of $\mathcal{L}_{\omega_{1} \omega}\left(\mathrm{BPA}_{\delta}^{\mathrm{fo}}\right)$. Observational equivalence is not definable in $\mathcal{L}\left(\mathrm{BPA}_{\delta}^{\mathrm{fo}}\right)$. It is shown in $[29]$ that external bisimilarity is not even definable in $\mathcal{L}_{\omega_{1} \omega}\left(\mathrm{BPA}_{\delta}^{\text {fo }}\right)$.

\section{SOS-Based Bisimilarity}

It is customary to associate transition systems with closed terms of the language of an ACP-like theory about processes by means of structural operational semantics and to identify closed terms if their associated transition systems are bisimilar. In this section, we briefly dwell on this approach.

In the presence of recursion the approach requires a special provision, namely constants for the solutions of recursive specifications.

We add to the nonlogical symbols of the first-order theory $\mathrm{BPA}_{\delta}^{\text {fo }}$, for each finite guarded recursive specification $E$ and each variable $X$ that occurs as the left-hand side of an equation in $E$, a constant standing for the unique solution of $E$ for $X$. This constant is denoted by $\langle X \mid E\rangle$. Moreover, we add the axiom (schema) given in Table 5 to the axioms of $\mathrm{BPA}_{\delta}^{\text {fo }}$. We write $\mathrm{BPA}_{\delta \mathrm{c}}^{\mathrm{fo}}$ for the resulting theory. RDPc is an axiom schema with an instance for each guarded recursive specification $E$. Note that the models of $\mathrm{BPA}_{\delta \mathrm{c}}^{\text {fo }}$ are simply the expansions of the models of $\mathrm{BPA}_{\delta}^{\text {fo }}$ obtained by associating with each constant $\langle X \mid E\rangle$ the unique solution in the model concerned of $E$ for $X$.

The structural operational semantics of $\mathrm{BPA}_{\delta \mathrm{c}}^{\mathrm{fo}}$ is described by the transition rules given in Table 6 . It determines a transition system for each process that can be denoted by a closed term of $\mathcal{L}\left(\mathrm{BPA}_{\delta \mathrm{c}}^{\mathrm{fo}}\right)$. These transition systems are special in the sense that their states are closed terms of $\mathcal{L}\left(\mathrm{BPA}_{\delta \mathrm{c}}^{\mathrm{fo}}\right)$.

Let $t$ be a closed term of $\mathcal{L}\left(\mathrm{BPA}_{\delta \mathrm{c}}^{\mathrm{fo}}\right)$. Then the transition system of $t$ induced by the structural operational semantics of $\mathrm{BPA}_{\delta \mathrm{c}}^{\mathrm{fo}}$, written $\mathrm{TS}(t)$, is the connected transition system $\Gamma\left(S, \rightarrow, \rightarrow \sqrt{ }, s^{0}\right)$, where:

$-S$ is the set of closed terms of $\mathcal{L}\left(\mathrm{BPA}_{\delta \mathrm{c}}^{\mathrm{fo}}\right)$;

- the sets $\stackrel{a}{\rightarrow} \subseteq S \times S$ and $\stackrel{a}{\rightarrow} \vee \subseteq S$ for each $a \in$ A are the smallest subsets of $S \times S$ and $S$, respectively, for which the transition rules from Table 6 hold; $-s^{0} \in S$ is $t$. 
Table 6. Structural Operational Semantics of $\mathrm{BPA}_{\delta \mathrm{c}}^{\text {fo }}$

$$
\begin{aligned}
& \stackrel{a}{a \stackrel{V}{ }} \\
& \frac{x \stackrel{a}{\rightarrow} \sqrt{ }}{x+y \stackrel{a}{\rightarrow} \sqrt{ }} \quad \frac{y \stackrel{a}{\rightarrow} \sqrt{ }}{x+y \stackrel{a}{\rightarrow} \sqrt{ }} \quad \frac{x \stackrel{a}{\rightarrow} x^{\prime}}{x+y \stackrel{a}{\rightarrow} x^{\prime}} \quad \frac{y \stackrel{a}{\rightarrow} y^{\prime}}{x+y \stackrel{a}{\rightarrow} y^{\prime}} \\
& \frac{x \stackrel{a}{\rightarrow} \sqrt{ }}{x \cdot y \stackrel{a}{\rightarrow} y} \quad \frac{x \stackrel{a}{\rightarrow} x^{\prime}}{x \cdot y \stackrel{a}{\rightarrow} x^{\prime} \cdot y} \\
& \frac{t_{i}\left(\left\langle X_{1} \mid E\right\rangle, \ldots,\left\langle X_{n} \mid E\right\rangle\right) \stackrel{a}{\rightarrow} \vee}{\left\langle X_{i} \mid E\right\rangle \stackrel{a}{\rightarrow} \vee} E=\left\{X_{i}=t_{i} \mid 1 \leq i \leq n\right\} \\
& \frac{t_{i}\left(\left\langle X_{1} \mid E\right\rangle, \ldots,\left\langle X_{n} \mid E\right\rangle\right) \stackrel{a}{\rightarrow} x^{\prime}}{\left\langle X_{i} \mid E\right\rangle \stackrel{a}{\rightarrow} x^{\prime}} E=\left\{X_{i}=t_{i} \mid 1 \leq i \leq n\right\}
\end{aligned}
$$

Clearly, the structural operational semantics does not give rise to infinitely branching transition systems. In other words, for each closed term $t$ of $\mathcal{L}\left(\mathrm{BPA}_{\delta \mathrm{c}}^{\text {fo }}\right)$, we have $\operatorname{TS}(t) \in \mathbb{T} \mathbb{S}_{\aleph_{0}}$.

Let $t_{1}$ and $t_{2}$ be closed terms of $\mathcal{L}\left(\mathrm{BPA}_{\delta \mathrm{c}}^{\text {fo }}\right)$. Then we say that $t_{1}$ and $t_{2}$ are

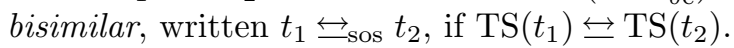

We have the following relationship between bisimilarity of terms, which is based on structural operational semantics, and validity of equation in models of $\mathrm{BPA}_{\delta \mathrm{c}}^{\mathrm{fo}}$.

\section{Theorem 8.1 (SOS-Based Bisimilarity and Validity of Equations).}

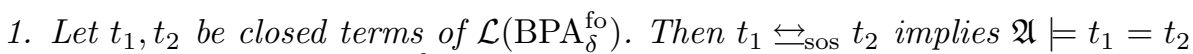
for all models $\mathfrak{A}$ of $\mathrm{BPA}_{\delta \mathrm{c}}^{\mathrm{fo}}$.

2. Let $t_{1}, t_{2}$ be closed terms of $\mathcal{L}\left(\mathrm{BPA}_{\delta \mathrm{c}}^{\mathrm{fo}}\right)$. Then $t_{1} \sharp_{\mathrm{sos}} t_{2}$ implies $\mathfrak{A} \models t_{1} \neq t_{2}$ for all models $\mathfrak{A}$ of $\mathrm{BPA}_{\delta \mathrm{c}}^{\text {fo }}$.

Proof.

Proof of part 1. It follows easily from the structural operational semantics of

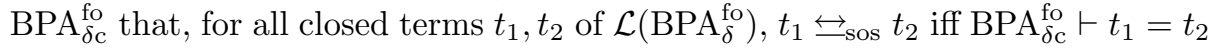
(see also [22]). From this, it follows immediately that, for all closed terms $t_{1}, t_{2}$

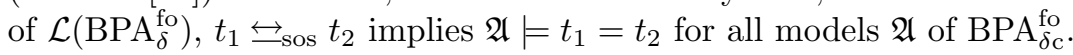

Proof of part 2. It follows easily from the structural operational semantics of

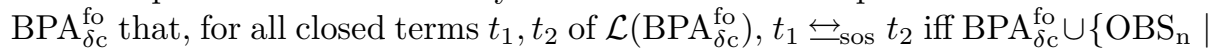
$n \geq 0\} \vdash t_{1} \approx_{n} t_{2}$ for all $n \geq 0$ (see also [22]). Moreover, for all closed terms $t_{1}, t_{2}$ of $\mathcal{L}\left(\mathrm{BPA}_{\delta \mathrm{c}}^{\text {fo }}\right)$ and $n \geq 0$, either $\mathrm{BPA}_{\delta \mathrm{c}}^{\text {fo }} \cup\left\{\mathrm{OBS}_{\mathrm{n}} \mid n \geq 0\right\} \vdash t_{1} \approx_{n} t_{2}$ or $\mathrm{BPA}_{\delta \mathrm{c}}^{\mathrm{fo}} \cup\left\{\mathrm{OBS}_{\mathrm{n}} \mid n \geq 0\right\} \vdash \neg t_{1} \approx_{n} t_{2}$, but not both. This is easily proved by induction on $n$. As a consequence, for all closed terms $t_{1}, t_{2}$ of $\mathcal{L}\left(\mathrm{BPA}_{\delta \mathrm{c}}^{\mathrm{fo}}\right)$, $t_{1} \sharp_{\text {sos }} t_{2}$ iff $\mathrm{BPA}_{\delta \mathrm{c}}^{\text {fo }} \cup\left\{\mathrm{OBS}_{\mathrm{n}} \mid n \geq 0\right\} \vdash \neg t_{1} \approx_{n} t_{2}$ for some $n \geq 0$. From this, it follows easily that, for all closed terms $t_{1}, t_{2}$ of $\mathcal{L}\left(\mathrm{BPA}_{\delta \mathrm{c}}^{\mathrm{fo}}\right), t_{1} \dddot{\sharp}_{\mathrm{sos}} t_{2}$ implies $\mathrm{BPA}_{\delta \mathrm{c}}^{\mathrm{fo}} \vdash \neg t_{1}=t_{2}$. From this, it follows immediately that, for all closed terms 
$t_{1}, t_{2}$ of $\mathcal{L}\left(\mathrm{BPA}_{\delta \mathrm{c}}^{\mathrm{fo}}\right), t_{1} \sharp_{\text {sos }} t_{2}$ implies $\mathfrak{A} \models \neg t_{1}=t_{2}$ for all models $\mathfrak{A}$ of $\mathrm{BPA}_{\delta \mathrm{c}}^{\text {fo }}$.

We could have introduced constants for the solutions of unguarded recursive specifications as well. In that case, the structural operational semantics would have given rise to countably branching transition systems. Moreover, it would have fixed a particular solution for each unguarded recursive specification. In this paper, we do not consider unguarded recursion.

The following remark on fixing a particular solution in the case of unguarded recursion is in order. Suppose that we also add to the nonlogical symbols of the first-order theory $\mathrm{BPA}_{\delta}^{\mathrm{fo}}$ a constant, denoted by $\langle X \mid E\rangle$, for each finite unguarded recursive specification $E$ and each variable $X$ that occurs as the lefthand side of an equation in $E$. Consider the two unguarded recursive specifications $X=a \cdot X+X$ and $Y=b \cdot Y+Y$, where $a$ and $b$ are different actions. The structural operational semantics of $\mathrm{BPA}_{\delta \mathrm{c}}^{\mathrm{fo}}$ described in Table 6 fixes the obvious solution for each of these unguarded recursive specifications. However, as usual with unguarded recursive specifications, both have more than one solution. The problem is not so much that they have more than one solution, but that the sets of solutions are not disjoint. For example, the solution of the guarded recursive specification $Z=a \cdot Z+b \cdot Z$ is a common solution of $X=a \cdot X+X$ and $Y=b \cdot Y+Y$. The common solutions exclude any possibility to prove that $\langle X \mid\{X=a \cdot X+X\}\rangle \neq\langle Y \mid\{Y=b \cdot Y+Y\}\rangle$, although $\langle X \mid\{X=a \cdot X+X\}\rangle \sharp_{\text {sos }}\langle Y \mid\{Y=b \cdot Y+Y\}\rangle$.

\section{A Modal Fragment of $\mathcal{L}\left(\mathrm{BPA}_{\delta}^{\text {fo }}\right)$}

In this section, we have a closer look at a modal fragment of $\mathcal{L}\left(\mathrm{BPA}_{\delta}^{\mathrm{fo}}\right)$. This fragment corresponds to a variant of HML (Hennessy-Milner Logic), a simple modal logic introduced in [23] to give a modal characterization of bisimilarity.

The set $\mathcal{M}$ of modal fragment formulas of $\mathcal{L}\left(\mathrm{BPA}_{\delta}^{\text {fo }}\right)$ is inductively defined as follows:

- if $x$ is a variable, then $x=x \in \mathcal{M}$;

- if $\phi \in \mathcal{M}$, then $\neg \phi \in \mathcal{M}$;

- if $\phi_{1}, \phi_{2} \in \mathcal{M}$, then $\phi_{1} \wedge \phi_{2} \in \mathcal{M}$;

- if $a \in \mathrm{A}$ and $x$ is a variable, then $x \stackrel{a}{\rightarrow} \sqrt{ } \in \mathcal{M}$;

- if $a \in \mathrm{A}, x, y$ are different variables and $\phi \in \mathcal{M}$, then $\exists y \bullet(x \stackrel{a}{\rightarrow} y \wedge \phi) \in \mathcal{M}$.

We write $\mathcal{M}_{1}$ for the subset of $\mathcal{M}$ that contains all formulas from $\mathcal{M}$ in which exactly one variable occurs free. The set $\mathcal{M}_{1}$ of one-variable modal fragment formulas has an interesting property: $\mathcal{M}_{1}$ is essentially the set of formulas of $\mathcal{L}\left(\mathrm{BPA}_{\delta}^{\text {fo }}\right)$ that are invariant for external bisimulation.

Theorem 9.1 (Invariance for External Bisimilarity). Let $\mathfrak{A}$ be a model of $\mathrm{BPA}_{\delta}^{\text {fo }}$ with domain $P$, and let $\phi$ be a formula of $\mathcal{L}\left(\mathrm{BPA}_{\delta}^{\text {fo }}\right)$. Then $\mathfrak{A} \models \phi\left[p_{1}\right]$ iff $\mathfrak{A}=\phi\left[p_{2}\right]$ for all $p_{1}, p_{2} \in P$ such that $p_{1} \overleftrightarrow{\mathfrak{A}}_{\mathfrak{A}} p_{2}$ iff there exists a formula $\phi^{\prime} \in \mathcal{M}_{1}$ such that $\phi \Leftrightarrow \phi^{\prime}$. 
Proof. The proof is analogous to the proof of the corresponding property for first-order formulas that correspond to HML-like modal formula given in [29].

We have the following corollary of Theorem 9.1.

Corollary 9.1 (External Bisimilarity Implies Indistinguishability). Let

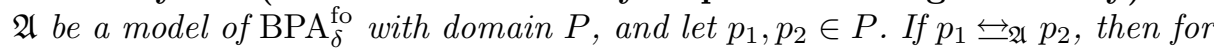
all $\phi \in \mathcal{M}_{1}$ we have $\mathfrak{A} \models \phi\left[p_{1}\right]$ iff $\mathfrak{A} \models \phi\left[p_{2}\right]$.

In general, we do not have the converse of Corollary 9.1. The transition systems from the counterexample used in the proof of Theorem 7.2 provide a counterexample here as well. However, we do have the converse in the case of finite branching.

Theorem 9.2 (Indistinguishability Implies External Bisimilarity). Let $\mathfrak{A}$ be a model of $\mathrm{BPA}_{\delta}^{\text {fo }}$ with domain $P$, and let $p_{1}, p_{2} \in P$. If for all $\phi \in \mathcal{M}_{1}$ we have $\mathfrak{A} \models \phi\left[p_{1}\right]$ iff $\mathfrak{A} \models \phi\left[p_{2}\right]$ and moreover $\operatorname{TS}\left(\mathfrak{A}, p_{1}\right), \operatorname{TS}\left(\mathfrak{A}, p_{2}\right) \in \mathbb{T S}_{\aleph_{0}}$, then

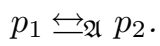

Proof. The proof is analogous to the proof of the corresponding property for HML-like modal formula given in [30].

Now we come back to the variant of HML of which the formulas correspond to the formulas in $\mathcal{M}$. HML is a modal logic introduced in [23] to be used in a setting where no distinction is made between successful termination and deadlock. The variant of HML considered here is adapted to a setting where distinction is made between successful termination and deadlock. This variant is henceforth also called HML. The set $\mathcal{H}$ of $H M L$ formulas is inductively defined as follows:

$-\mathrm{T} \in \mathcal{H}$

- if $\psi \in \mathcal{H}$, then $\neg \psi \in \mathcal{H}$;

- if $\psi_{1}, \psi_{2} \in \mathcal{H}$, then $\psi_{1} \wedge \psi_{2} \in \mathcal{H}$

- if $a \in \mathrm{A}$, then $\langle a\rangle \vee \in \mathcal{H}$;

- if $a \in \mathrm{A}$ and $\psi \in \mathcal{H}$, then $\langle a\rangle \psi \in \mathcal{H}$.

There is a "standard translation" from HML formulas to formulas of $\mathcal{L}\left(\mathrm{BPA}_{\delta}^{\text {fo }}\right)$. Let $x$ be a fixed but arbitrary variable. Then the translation of HML formulas is defined as follows:

$$
\begin{array}{ll}
\mathrm{T}^{\bullet} & =x=x, \\
(\neg \psi)^{\bullet} & =\neg\left(\psi^{\bullet}\right), \\
\left(\psi_{1} \wedge \psi_{2}\right)^{\bullet} & =\psi_{1} \bullet \wedge \psi_{2} \bullet \\
\langle a\rangle \vee^{\bullet} & =x \stackrel{a}{\rightarrow} \sqrt{ }, \\
(\langle a\rangle \psi)^{\bullet} & =\exists y \bullet\left(x \stackrel{a}{\rightarrow} y \wedge \psi^{\bullet}(y)\right) \quad \text { where } y \text { is a fresh variable. }
\end{array}
$$

This translation is justified by the fact that satisfaction for HML formulas $\psi$ is defined such that $\mathfrak{A}=\psi$ iff $\mathfrak{A}=\forall x \bullet \psi \bullet$ 
Table 7. Axioms for Deadlock Freedom

\begin{tabular}{lr}
\hline$\neg \operatorname{dlf}(\delta)$ & DLF1 \\
$\bigwedge_{a \in \mathrm{A}} \forall x, y \bullet(\operatorname{dlf}(x) \wedge x \stackrel{a}{\rightarrow} y \Rightarrow \operatorname{dlf}(y))$ & DLF2 \\
$\neg \psi(\delta) \wedge \bigwedge_{a \in \mathrm{A}} \forall x, y \bullet(\psi(x) \wedge x \stackrel{a}{\rightarrow} y \Rightarrow \psi(y)) \Rightarrow \forall x \bullet(\psi(x) \Rightarrow \operatorname{dlf}(x))$ & DLFS \\
\hline
\end{tabular}

Clearly, the image of the translation from HML formulas to formulas of $\mathcal{L}\left(\mathrm{BPA}_{\delta}^{\text {fo }}\right)$ consists of all formulas from $\mathcal{M}_{1}$ of which the free variable is $x$. HML is a modal logic which has been devised to complement the process algebra CCS $[26,27]$ with a formalism that admits to express and verify properties of processes which are definable directly in terms of the action steps that are possible at any stage. Apparently, $\mathrm{BPA}_{\delta}^{\text {fo }}$ can be considered to include a process algebra and a variant of HML as fragments.

\section{Deadlock Freedom}

In this section, we add a deadlock freedom predicate to $\mathrm{BPA}_{\delta}^{\text {fo }}$. At the end of Section 2, we demonstrated that the deadlock freedom predicate can be explicitly defined by using the reachability predicate. Here, the deadlock freedom predicate will be implicitly defined without using the reachability predicate.

We add to $\mathrm{BPA}_{\delta}^{\text {fo }}$ the unary deadlock freedom predicate symbol dif and the axioms given in Table 7. We write DLF for this set of axioms. Axiom DLFS is actually an axiom schema: $\psi$ stands for an arbitrary formula of $\mathcal{L}\left(\mathrm{BPA}_{\delta}^{\text {fo }} \cup \mathrm{DLF}\right)$ in which only the variable $x_{1}$ may have free occurrences. The notation $\psi\left(t_{1}\right)$ is used for the formula obtained from $\psi$ by replacing all free occurrences of $x_{1}$ by $t_{1}$. Axiom schema DLFS is an induction schema.

The deadlock freedom predicate that is implicitly defined by DLF is equivalent to the one that is explicitly defined by using the reachability predicate.

Theorem 10.1 (Explicit Definability of Deadlock Freedom). We have $\mathrm{BPA}_{\delta}^{\text {fo }} \cup \operatorname{DLF} \vdash \operatorname{dlf}(x) \Leftrightarrow \neg x \rightarrow \delta$.

Proof. We will apply $\mathrm{RS}$, taking $\operatorname{dlf}(x) \Rightarrow y \neq \delta$ for $\phi$, to prove the implication $\operatorname{dlf}(x) \Rightarrow \neg x \rightarrow \delta$. When we have shown that $x \rightarrow y \Rightarrow(\operatorname{dlf}(x) \Rightarrow y \neq \delta)$, we can first conclude by substitution of $\delta$ for $y$ that $x \rightarrow \delta \Rightarrow \neg \operatorname{dlf}(x)$, and then by contraposition that $\operatorname{dIf}(x) \Rightarrow \neg x \rightarrow \delta$.

It remains to be shown by means of RS that $x \rightarrow y \Rightarrow(\operatorname{dlf}(x) \Rightarrow y \neq \delta)$. First of all, we immediately conclude from DLF1 that

$$
\forall x^{\prime} \bullet\left(\operatorname{dlf}\left(x^{\prime}\right) \Rightarrow x^{\prime} \neq \delta\right) .
$$

Moreover, we conclude from DLF2, using substitutivity of implication, that

$$
\forall x^{\prime}, y^{\prime}, z^{\prime} \bullet \bigwedge_{a^{\prime} \in \mathrm{A}}\left(x^{\prime} \stackrel{a^{\prime}}{\longrightarrow} y^{\prime} \wedge\left(\operatorname{dlf}\left(y^{\prime}\right) \Rightarrow z^{\prime} \neq \delta\right) \Rightarrow\left(\operatorname{dlf}\left(x^{\prime}\right) \Rightarrow z^{\prime} \neq \delta\right)\right) .
$$


Using the subprocess induction schema, it follows from these conclusions that $x \rightarrow y \Rightarrow(\operatorname{dlf}(x) \Rightarrow y \neq \delta)$.

We will apply DLFS, taking $\neg x \rightarrow \delta$ for $\psi$, to prove the reverse implication $\neg x \rightarrow \delta \Rightarrow \operatorname{dlf}(x)$.

First of all, we immediately conclude from R1 that

$$
\neg(\neg \delta \rightarrow \delta) .
$$

Moreover, we conclude from R2, because $(x \stackrel{a}{\rightarrow} y \wedge y \rightarrow z \Rightarrow x \rightarrow z) \Leftrightarrow$ $(\neg x \rightarrow z \wedge x \stackrel{a}{\rightarrow} y \Rightarrow \neg y \rightarrow z)$, that

$$
\bigwedge_{a \in \mathrm{A}} \forall x, y \bullet(\neg x \rightarrow \delta \wedge x \stackrel{a}{\rightarrow} y \Rightarrow \neg y \rightarrow \delta) .
$$

Using DLFS, it follows from these conclusions that $\forall x \bullet(\neg x \rightarrow \delta \Rightarrow \operatorname{dlf}(x))$.

Using Proposition 2.1 and Theorem 10.1, we can easily prove that, for example, the solution of the guarded recursive specification $X=a \cdot X$ is deadlock free.

Proposition 10.1 (Solution of $X=a \cdot X$ is Deadlock Free). We have $\mathrm{BPA}_{\delta}^{\text {fo }} \cup \operatorname{DLF} \vdash X=a \cdot X \Rightarrow \operatorname{dlf}(X)$.

Proof. Suppose $\neg \operatorname{dlf}(X)$. By Theorem 10.1, then also $X \rightarrow \delta$. We distinguish three cases according to Proposition 2.1:

- $X=\delta$. Then, because $X=a \cdot X$, also $\delta=a \cdot \delta$. This is equivalent to $a \cdot \delta \sqsubseteq \delta$. However, $\neg a \cdot \delta \sqsubseteq \delta$. Contradiction.

- $X \stackrel{a}{\rightarrow} \delta$ for some $a \in \mathrm{A}$. Then $a \cdot \delta \sqsubseteq X$. Because $X=a \cdot X$, this is equivalent to $a \cdot \delta \sqsubseteq a \cdot X$. This in turn implies $\delta=X$. We already know from the previous case that $X \neq \delta$. Contradiction.

$-X \stackrel{a}{\rightarrow} z$ for some $a \in \mathrm{A}$ and $z \neq X$ with $z \rightarrow \delta$. Then $a \cdot z \sqsubseteq X$. Because $X=a \cdot X$, this is equivalent to $a \cdot z \sqsubseteq a \cdot X$. This in turn implies $z=X$. However, $z \neq X$. Contradiction.

So $\neg \operatorname{dlf}(X)$ leads in all cases to contradiction. From this, we conclude that $\operatorname{dlf}(X)$.

Let $\mathfrak{A}$ be a model of $\mathrm{BPA}_{\delta}^{\text {fo }} \cup$ DLF with domain $P$. Then reachability and deadlock freedom on $P$ are defined as follows:

$$
p_{1} \rightarrow \mathfrak{A} p_{2} \quad \text { iff } \quad p_{1} \rightarrow p_{2},
$$

where $\rightarrow$ is the reachability relation of $\operatorname{TS}\left(\mathfrak{A}, p_{1}\right)$;

$$
\operatorname{dlf}_{\mathfrak{A}}(p) \text { iff } \operatorname{not} p \rightarrow \mathfrak{A} \delta^{\mathfrak{A}} .
$$

Reachability and deadlock freedom on the domain of a model of $\mathrm{BPA}_{\delta}^{\text {fo }} \cup$ DLF as defined above are called external reachability and external deadlock freedom, respectively.

In the models of $\mathrm{BPA}_{\delta}^{\text {fo }} \cup \mathrm{DLF}$, internal deadlock freedom implies external deadlock freedom. 
Proposition 10.2 (Internal Deadlock Freedom Implies External Deadlock Freedom). Let $\mathfrak{A}$ be a model of $\mathrm{BPA}_{\delta}^{\mathrm{fo}} \cup \mathrm{DLF}$ with domain $P$ and let $p \in P$. Then $\mathrm{dlf}^{\mathfrak{A}}(p)$ implies $\operatorname{dlf}_{\mathfrak{A}}(p)$.

Proof. By Theorem 10.1, dlf $\mathfrak{A}^{\mathfrak{A}}(p)$ iff not $p \rightarrow^{\prime} \delta^{\mathfrak{A}}$, where $\rightarrow^{\prime}$ is the binary relation on $P$ associated with the predicate symbol $\rightarrow$ in $\mathfrak{A}$. By the definition of external deadlock freedom, $\operatorname{dlf}_{\mathfrak{A}}(p)$ iff not $p \rightarrow^{\prime \prime} \delta^{\mathfrak{A}}$, where $\rightarrow^{\prime \prime}$ is the reachability relation of $\operatorname{TS}(\mathfrak{A}, p)$. It follows immediately from axioms R1, R2 and RS of $\mathrm{BPA}_{\delta}^{\mathrm{fo}}$ (Table 1) and the definition of reachability relation of a transition system (Section 4) that for all $p^{\prime}, p^{\prime \prime} \in P, p^{\prime} \rightarrow^{\prime \prime} p^{\prime \prime}$ implies $p^{\prime} \rightarrow^{\prime} p^{\prime \prime}$. Hence, $p \rightarrow{ }^{\prime \prime} \delta^{\mathfrak{A}}$ implies $p \rightarrow^{\prime} \delta^{\mathfrak{A}}$; and by the above-mentioned equivalences $\operatorname{dlf}^{\mathfrak{A}}(p)$ implies $\operatorname{dlf}_{\mathfrak{A}}(p)$.

We write $\mathfrak{P}_{\kappa}^{\text {dlf }}\left(\kappa \geq \aleph_{0}\right)$ for the unique definitional expansion of $\mathfrak{P}_{\kappa}$ determined by the definitional extension of $\mathrm{BPA}_{\delta}^{\text {fo }}$ with axioms DLF1, DLF2 and DLFS. In the full bisimulation models $\mathfrak{P}_{\kappa}^{\text {dlf }}$, external deadlock freedom coincides with internal deadlock freedom.

Proposition 10.3 (External Deadlock Freedom Is Internal Deadlock Freedom in $\left.\mathfrak{P}_{\kappa}^{\text {dlf }}\right)$. Let $p \in \mathbb{T} \mathbb{S}_{\kappa} / \leftrightarrows$ for some $\kappa \geq \aleph_{0}$. Then $\operatorname{dlf}_{\mathfrak{P}_{\kappa}^{\text {dlf }}}(p)$ iff $\widetilde{\operatorname{dlf}}(p)$.

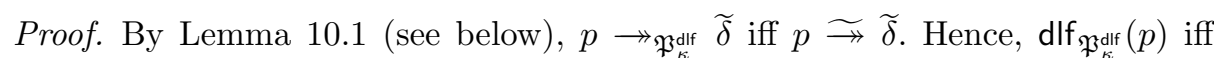
not $p \widetilde{\rightarrow} \widetilde{\delta}$. By Theorem 10.1, also dlf $(p)$ iff not $p \widetilde{\rightarrow} \widetilde{\delta}$. From this, it follows immediately that $\mathrm{dlf}_{\mathfrak{P}_{k}^{\text {dlf }}}(p)$ iff $\widetilde{\operatorname{dlf}}(p)$.

In the above proof, we have used the next lemma. It states that in the full bisimulation models $\mathfrak{P}_{\kappa}^{\text {dif }}$, external reachability coincides with internal reachability.

Lemma 10.1 (External Reachability Is Internal Reachability in $\mathfrak{P}_{\kappa}^{\text {dlf }}$ ). Let $p_{1}, p_{2} \in \mathbb{T S}_{\kappa} / \leftrightarrows$ for some $\kappa \geq \aleph_{0}$. Then $p_{1} \rightarrow \mathfrak{P}_{\kappa}^{\text {diff }} p_{2}$ iff $p_{1} \underset{\rightarrow}{\longrightarrow} p_{2}$.

Proof. By Lemma 6.1, $\mathrm{TS}\left(\mathfrak{P}_{\kappa}, p_{1}\right) \in p_{1}$ and $\operatorname{TS}\left(\mathfrak{P}_{\kappa}, p_{2}\right) \in p_{2}$. Hence, $p_{1} \widetilde{\rightarrow} p_{2}$ iff $\left[\mathrm{TS}\left(\mathfrak{P}_{\kappa}, p_{1}\right)\right] \widetilde{\longrightarrow}\left[\mathrm{TS}\left(\mathfrak{P}_{\kappa}, p_{2}\right)\right]$. It is easy to see that $p$ is a state of $\operatorname{TS}\left(\mathfrak{P}_{\kappa}, p_{1}\right)$ iff $p_{1} \rightarrow p$ where $\rightarrow$ is the reachability relation of $\operatorname{TS}\left(\mathfrak{P}_{\kappa}, p_{1}\right)$; and also that, if $p$ is a state of $\operatorname{TS}\left(\mathfrak{P}_{\kappa}, p_{1}\right),\left(\operatorname{TS}\left(\mathfrak{P}_{\kappa}, p_{1}\right)\right)_{p}=\operatorname{TS}\left(\mathfrak{P}_{\kappa}, p\right)$. From this, and the definitions of $\widetilde{\rightarrow}$ and $\widehat{\rightarrow}$, it follows that $\left[\operatorname{TS}\left(\mathfrak{P}_{\kappa}, p_{1}\right)\right] \stackrel{\rightarrow}{\longrightarrow}\left[\operatorname{TS}\left(\mathfrak{P}_{\kappa}, p_{2}\right)\right]$ iff there exists a $p$ such that $p_{1} \rightarrow \mathfrak{P}_{\kappa} p$ and $\operatorname{TS}\left(\mathfrak{P}_{\kappa}, p\right) \in\left[\mathrm{TS}\left(\mathfrak{P}_{\kappa}, p_{2}\right)\right]$. Moreover, by Lemma 6.1, $\mathrm{TS}\left(\mathfrak{P}_{\kappa}, p\right) \in\left[\mathrm{TS}\left(\mathfrak{P}_{\kappa}, p_{2}\right)\right]$ iff $p=p_{2}$. Thus, we conclude that $p_{1} \underset{\rightarrow}{\widetilde{\longrightarrow}} p_{2}$ iff $p_{1} \rightarrow \mathfrak{P}_{\kappa}$ $p_{2}$. Because $\mathfrak{P}_{\kappa}^{\text {dlf }}$ is a definitional expansion of $\mathfrak{P}_{\kappa}$, it follows immediately that

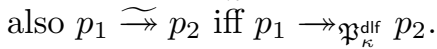

A useful corollary of the proof of Lemma 10.1 is the following.

Corollary 10.1 (External Reachability Is Internal Reachability in $\mathfrak{P}_{\kappa}$ ). Let $p_{1}, p_{2} \in \mathbb{T S}_{\kappa} / \leftrightarrows$ for some $\kappa \geq \aleph_{0}$. Then $p_{1} \rightarrow \mathfrak{P}_{\kappa} p_{2}$ iff $p_{1} \underset{\rightarrow}{\overparen{ }} p_{2}$.

There does not exist a consistent extension of $\mathrm{BPA}_{\delta}^{\text {fo }} \cup$ DLF with first-order axioms that has only models in which external deadlock freedom coincides with internal deadlock freedom. 
Theorem 10.2 (Undefinability of External Deadlock Freedom). External deadlock freedom is not first-order definable in any consistent extension of $\mathrm{BPA}_{\delta}^{\text {fo }} \cup \mathrm{DLF}$.

Proof. From Proposition 10.3, we know that it is sufficient to show that each first-order consistent extension of $\mathrm{BPA}_{\delta}^{\text {fo }} \cup \mathrm{DLF}$ has a model in which external deadlock freedom is not internal deadlock freedom.

Suppose that each first-order consistent extension of $\mathrm{BPA}_{\delta}^{\text {fo }} \cup \mathrm{DLF}$ has only models in which external deadlock freedom is internal deadlock freedom. A contradiction is found as follows. Let $c_{0}, c_{1}, c_{2}, \ldots$ be different new constants; and let $a$ be an action. Consider the following set of formulas:

$$
H=\left\{\neg \operatorname{dlf}\left(c_{0}\right)\right\} \cup\left\{c_{i}=a \cdot c_{i+1} \mid i \geq 0\right\} .
$$

Each finite $H^{\prime} \subseteq H$ is satisfiable in an expansion of $\mathfrak{P}_{\aleph_{0}}^{\text {dlf }}$. Hence, each finite $H^{\prime} \subseteq H$ is consistent with $\mathrm{BPA}_{\delta}^{\text {fo }} \cup \mathrm{DLF}$. From this, it follows by the Compactness Theorem that $H$ is consistent with $\mathrm{BPA}_{\delta}^{\text {fo }} \cup$ DLF. Now consider an arbitrary model $\mathfrak{A}$ of $\mathrm{BPA}_{\delta}^{\text {fo }} \cup \mathrm{DLF} \cup H$. It is easy to see that the reachability relation $\rightarrow$ of $\operatorname{TS}\left(\mathfrak{A}, c_{0}^{\mathfrak{A}}\right)$ is such that not $c_{0}^{\mathfrak{A}} \rightarrow \delta^{\mathfrak{A}}$. Hence, $\operatorname{dlf}_{\mathfrak{A}}\left(c_{0}^{\mathfrak{A}}\right)$. However, because $\mathfrak{A} \models \neg \operatorname{dlf}\left(c_{0}\right)$, not $\operatorname{dlf}^{\mathfrak{A}}\left(c_{0}^{\mathfrak{A}}\right)$.

Apparently, there is a discrepancy in relation to deadlock freedom which is similar to the discrepancy in relation to bisimilarity found in Section 6 .

We can summarize the state of affairs as follows. Deadlock freedom derivable from $\mathrm{BPA}_{\delta}^{\text {fo }} \cup \mathrm{DLF}$ implies external deadlock freedom in each model of $\mathrm{BPA}_{\delta}^{\text {fo }} \cup$ DLF. In the full bisimulation models $\mathfrak{P}_{\kappa}^{\text {dlf }}$, external deadlock freedom coincides with internal deadlock freedom. However, there also exist models of which the domain contains elements that are externally deadlock free, but not internally deadlock free. Moreover, those models cannot be excluded by extending $\mathrm{BPA}_{\delta}^{\text {fo }} \cup$ DLF with first-order axioms.

\section{Determinism}

In the previous section, the relation between external deadlock freedom and internal deadlock freedom in models of $\mathrm{BPA}_{\delta}^{\text {fo }} \cup \mathrm{DLF}$ was analysed in detail. It is obvious that there are other properties of processes of which the relation between the external version and the internal version can be analysed. In this section, we briefly consider one other property, namely determinism.

The determinism predicate symbol det is explicitly defined in terms of $\mathcal{L}\left(\mathrm{BPA}_{\delta}^{\text {fo }}\right)$ by

$$
\begin{aligned}
& \operatorname{det}(x) \Leftrightarrow \forall y \bullet\left(x \rightarrow y \Rightarrow \bigwedge_{a \in \mathrm{A}}((y \stackrel{a}{\rightarrow} \vee \Rightarrow \forall z \bullet \neg y \stackrel{a}{\rightarrow} z) \wedge\right. \\
& \left.\left.\forall z, z^{\prime} \bullet\left(y \stackrel{a}{\longrightarrow} z \wedge y \stackrel{a}{\longrightarrow} z^{\prime} \Rightarrow z=z^{\prime}\right)\right)\right) .
\end{aligned}
$$

External determinism can be defined in the same vein as external deadlock freedom. 
Table 8. First-Order and Second-Order Axioms for Restricted Reachability

\begin{tabular}{lr}
\hline$x \stackrel{a}{\rightarrow} x$ & RR1 \\
$x \stackrel{a}{\rightarrow} y \wedge y \stackrel{a}{\rightarrow} z \Rightarrow x \stackrel{a}{\rightarrow} z$ & RR2 \\
$\exists ! y \bullet \psi^{a, b}(x, y)$ & RR3 \\
$x \stackrel{a}{\rightarrow} y \wedge$ & \\
$\forall x^{\prime}, y^{\prime}, z^{\prime} \bullet\left(\phi\left(x^{\prime}, x^{\prime}\right) \wedge\left(x^{\prime} \stackrel{a}{\rightarrow} y^{\prime} \wedge \phi\left(y^{\prime}, z^{\prime}\right) \Rightarrow \phi\left(x^{\prime}, z^{\prime}\right)\right)\right) \Rightarrow \phi(x, y)$ & RRS \\
$\forall R \bullet(x \stackrel{a}{\rightarrow} y \wedge$ & \\
$\left.\quad \forall x^{\prime}, y^{\prime}, z^{\prime} \bullet\left(R\left(x^{\prime}, x^{\prime}\right) \wedge\left(x^{\prime} \stackrel{a}{\rightarrow} y^{\prime} \wedge R\left(y^{\prime}, z^{\prime}\right) \Rightarrow R\left(x^{\prime}, z^{\prime}\right)\right)\right) \Rightarrow R(x, y)\right)$ & $\mathrm{RR}$ \\
\hline
\end{tabular}

In this case, it is easy to see that there exist models of the extension of $\mathrm{BPA}_{\delta}^{\text {fo }}$ with determinism in which external determinism does not coincide with internal determinism. We know from Theorem 6.1 that each first-order extension of $\mathrm{BPA}_{\delta}^{\text {fo }}$ has a model of which the domain contains pairs of different elements that are externally bisimilar. Let $\mathfrak{A}$ be such a model, and let $p$ and $p^{\prime}$ be elements

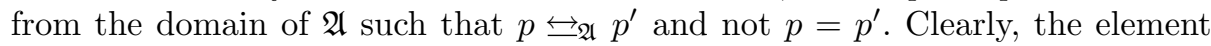
$a^{\mathfrak{A}} \cdot \mathfrak{A} p+{ }^{\mathfrak{A}} a^{\mathfrak{A}} \cdot \mathfrak{A} p^{\prime}$ is externally deterministic, but not internally deterministic.

It is also easy to see that external determinism coincides with internal determinism in the unique expansions of the full bisimulation models $\mathfrak{P}_{\kappa}$ determined by the explicit definition of det. We know from Proposition 6.1 that external bisimilarity coincides with identity in those models; and we know from Corollary 10.1 that external reachability coincides with internal reachability in those models. From this, it is clear that external determinism coincides with internal determinism in those models.

\section{Restricted Reachability}

In this section, we present an interesting extension of $\mathrm{BPA}_{\delta}^{\mathrm{fo}}$, called $\mathrm{BPA}_{\delta \mathrm{rr}}^{\mathrm{fo}}$. It is obtained as follows. We add to the nonlogical symbols of $\mathrm{BPA}_{\delta}^{\text {fo }}$, for each $a \in \mathrm{A}$, a binary reachability by a-steps predicate symbol $\stackrel{a}{\rightarrow}$. Morover, we add the axioms given in Table 8, with the exception of the second-order axiom RR, to the axioms of $\mathrm{BPA}_{\delta}^{\mathrm{fo}}$. In axiom RR3 and henceforth, $\psi^{a, b}(x, y)$, where $a$ and $b$ are different actions, stands for the formula

$$
\begin{aligned}
& y \stackrel{b}{\rightarrow} x \wedge \forall \bar{x} \bullet(y \stackrel{b}{\rightarrow} \bar{x} \Rightarrowx=\bar{x}) \wedge \\
& \forall y^{\prime} \bullet\left(y \stackrel{a}{\longrightarrow} y^{\prime} \Rightarrow \exists x^{\prime}, x^{\prime \prime} \bullet\right.\left(y^{\prime} \stackrel{b}{\rightarrow} x^{\prime} \wedge \bigvee_{a^{\prime} \in \mathrm{A}} x^{\prime} \stackrel{a^{\prime}}{\longrightarrow} x^{\prime \prime} \Rightarrow\right. \\
&\left.\exists ! y^{\prime \prime} \bullet\left(y^{\prime} \stackrel{a}{\longrightarrow} y^{\prime \prime} \wedge y^{\prime \prime} \stackrel{b}{\rightarrow} x^{\prime \prime}\right)\right) \wedge \\
& \exists x^{\prime}, y^{\prime \prime} \bullet \\
&\left(y^{\prime} \stackrel{b}{\rightarrow} x^{\prime} \wedge y^{\prime} \stackrel{a}{\longrightarrow} y^{\prime \prime} \Rightarrow\right. \\
&\left.\left.\exists ! x^{\prime \prime} \bullet\left(\bigvee_{a^{\prime} \in \mathrm{A}} x^{\prime} \stackrel{a^{\prime}}{\longrightarrow} x^{\prime \prime} \wedge y^{\prime \prime} \stackrel{b}{\rightarrow} x^{\prime \prime}\right)\right)\right) .
\end{aligned}
$$


RR1-RR3 are actually axiom schemas in which $a$ and $b$ stand for arbitrary different actions. RRS is actually an axiom schema in which $a$ stands for an arbitrary action and $\phi$ for an arbitrary formula of $\mathcal{L}\left(\mathrm{BPA}_{\delta \mathrm{rr}}^{\mathrm{fo}}\right)$ in which only the variables $x_{1}$ and $x_{2}$ may have free occurrences. The differences of RR1, RR2 and RRS with R1, R2 and RS reflect that $\stackrel{a}{\rightarrow}$ is the restricted kind of reachability in which only action $a$ is involved. We will return to the additional axiom schema RR3 below. Axiom schema RRS is called the restricted subprocess induction schema.

Similar to RS, the first-order axiom schema RRS does not exclude all models in which there are processes that have more processes reachable by $a$-steps than needed to satisfy the instances of axiom schemas RR1 and RR2. Similar to R, the second-order axiom schema RR from Table 8, in which $a$ stands for an arbitrary action, would exclude all such models.

One can think of $\psi^{a, b}(x, y)$ as a formula expressing that $y$ produces an indexing of the processes reachable from $x$ with a set of processes reachable from $y$ by $a$-steps only. Axiom schema RR3 excludes models in which such an indexing cannot be produced for all processes. This is indispensable to establish that the (unrestricted) reachability predicate is explicitly definable by means of a restricted reachability predicate. It is unknown to us whether RR3 is derivable from the other axioms of $\mathrm{BPA}_{\delta \mathrm{rr}}^{\mathrm{fo}}$.

Note further that axiom schema RR3 induces the existence of an indexing operator for each pair of different actions $a$ and $b$. The formula

$$
\chi_{a, b}(x)=y \Leftrightarrow \psi^{a, b}(x, y)
$$

is an explicit definition of this operator in terms of $\mathcal{L}\left(\mathrm{BPA}_{\delta \mathrm{rr}}^{\mathrm{fo}}\right)$. Thus, a definitional extension of $\mathrm{BPA}_{\delta \mathrm{rr}}^{\mathrm{fo}}$ is obtained. Hence, every model of $\mathrm{BPA}_{\delta \mathrm{rr}}^{\text {fo }}$ can be expanded in a unique way with an indexing operation that satisfies this formula. Using an auxiliary operator $\bar{\chi}_{a, b}$, we can equationally characterize $\chi_{a, b}$ as follows:

$$
\begin{aligned}
& \chi_{a, b}(x)=b \cdot x+\bar{\chi}_{a, b}(x), \\
& \bar{\chi}_{a, b}(c)=\delta, \\
& \bar{\chi}_{a, b}(c \cdot x)=a \cdot \chi_{a, b}(x), \\
& \bar{\chi}_{a, b}(x+y)=\bar{\chi}_{a, b}(x)+\bar{\chi}_{a, b}(x),
\end{aligned}
$$

where $c$ stands for an arbitrary constant of $\mathrm{BPA}_{\delta \mathrm{rr}}^{\text {fo }}$ (i.e. $c \in \mathrm{A} \cup\{\delta\}$ ).

Now we come back to the explicit definability of unrestricted reachability.

Theorem 12.1 (Explicit Definability of Unrestricted Reachability). We have $\mathrm{BPA}_{\delta \mathrm{rr}}^{\mathrm{fo}} \vdash x \rightarrow y \Leftrightarrow \mathrm{P}_{\rightarrow}(x, y)$, where $\mathrm{P}_{\rightarrow}(x, y)$ stands for the following formula of $\mathcal{L}\left(\mathrm{BPA}_{\delta \mathrm{rr}}^{\mathrm{fo}}\right)$ :

$$
\exists z \bullet\left(\psi^{a, b}(x, z) \wedge \exists z^{\prime} \bullet\left(z \stackrel{a}{\longrightarrow} z^{\prime} \wedge z^{\prime} \stackrel{b}{\rightarrow} y\right)\right),
$$

with $a$ and $b$ different actions. 
Proof. We will apply the subprocess induction schema RS, taking $\mathrm{P}_{\rightarrow}(x, y)$ for $\phi$, to prove the implication $x \rightarrow y \Rightarrow \mathrm{P}_{\rightarrow}(x, y)$.

First of all, we conclude from RR1 and RR3, because $\psi^{a, b}(x, z) \Rightarrow z \stackrel{b}{\rightarrow} x$, that

$$
\forall x^{\prime} \bullet \mathrm{P}_{\rightarrow}\left(x^{\prime}, x^{\prime}\right) .
$$

Moreover, using SI1, SI9, TR2 and RR2, we easily derive the following:

$$
\begin{aligned}
& x^{\prime} \stackrel{a^{\prime}}{\longrightarrow} y^{\prime} \wedge \psi^{a, b}\left(y^{\prime}, u^{\prime}\right) \wedge \exists u^{\prime \prime} \bullet\left(u^{\prime} \stackrel{a}{\longrightarrow} u^{\prime \prime} \wedge u^{\prime \prime} \stackrel{b}{\rightarrow} z^{\prime}\right) \Rightarrow \\
& \psi^{a, b}\left(x^{\prime}, a \cdot u^{\prime}+b \cdot x^{\prime}\right) \wedge \exists u^{\prime \prime} \bullet\left(a \cdot u^{\prime}+b \cdot x^{\prime} \stackrel{a}{\longrightarrow} u^{\prime \prime} \wedge u^{\prime \prime} \stackrel{b}{\rightarrow} z^{\prime}\right) .
\end{aligned}
$$

Hence, we conclude from RR3, using existential generalization, that

$$
\forall x^{\prime}, y^{\prime}, z^{\prime} \cdot \bigwedge_{a^{\prime} \in \mathrm{A}}\left(x^{\prime} \stackrel{a^{\prime}}{\longrightarrow} y^{\prime} \wedge \mathrm{P}_{\rightarrow}\left(y^{\prime}, z^{\prime}\right) \Rightarrow \mathrm{P}_{\rightarrow}\left(x^{\prime}, z^{\prime}\right)\right) .
$$

Using the subprocess induction schema, it follows from these conclusions that $x \rightarrow y \Rightarrow \mathrm{P}_{\rightarrow}(x, y)$.

In the proof of the implication $\mathrm{P}_{\rightarrow}(x, y) \Rightarrow x \rightarrow y$ given below, $\rho\left(u, u^{\prime}\right)$ stands for the formula

$$
\begin{aligned}
& \exists x \bullet \psi^{a, b}(x, u) \Rightarrow \\
& \quad \exists ! x \bullet\left(\psi^{a, b}(x, u) \wedge u \stackrel{a}{\rightarrow} u^{\prime} \wedge \exists ! y \bullet\left(u^{\prime} \stackrel{b}{\rightarrow} y \wedge x \rightarrow y\right)\right) .
\end{aligned}
$$

We will apply the restricted subprocess induction schema RRS, taking $\rho\left(u, u^{\prime}\right)$ for $\phi$. When we have shown in this manner that $u \stackrel{a}{\longrightarrow} u^{\prime} \Rightarrow \rho\left(u, u^{\prime}\right)$, we can conclude that $\mathrm{P}_{\rightarrow}(x, y) \Rightarrow x \rightarrow y$ as follows. Assume $\mathrm{P}_{\rightarrow}(x, y)$. Then there exist $u$ and $u^{\prime}$ such that $\psi^{a, b}(x, u) \wedge u \stackrel{a}{\rightarrow} u^{\prime} \wedge u^{\prime} \stackrel{b}{\rightarrow} y$. Because $u \stackrel{a}{\rightarrow} u^{\prime} \Rightarrow \rho\left(u, u^{\prime}\right)$, also $\rho\left(u, u^{\prime}\right)$. This immediately gives $x \rightarrow y$.

It remains to be shown by means of RRS that $u \stackrel{a}{\rightarrow} u^{\prime} \Rightarrow \rho\left(u, u^{\prime}\right)$. First of all, we conclude from RR1 and R1, because $\psi^{a, b}(x, u) \Rightarrow u \stackrel{b}{\rightarrow} x$, that

$$
\forall u \bullet \rho(u, u) \text {. }
$$

Moreover, using RR2 and R2, we easily derive from the hypothesis $\exists x \bullet \psi^{a, b}(x, u)$ the following implications:

$$
\begin{aligned}
& u \stackrel{a}{\rightarrow} u^{\prime} \wedge \psi^{a, b}\left(x^{\prime}, u^{\prime}\right) \Rightarrow \exists ! x \bullet\left(\psi^{a, b}(x, u) \wedge \bigvee_{a^{\prime} \in \mathrm{A}} x \stackrel{a^{\prime}}{\rightarrow} x^{\prime}\right), \\
& u \stackrel{a}{\longrightarrow} u^{\prime} \wedge u^{\prime} \stackrel{a}{\rightarrow} u^{\prime \prime} \Rightarrow u \stackrel{a}{\rightarrow} u^{\prime \prime}, \\
& \bigvee_{a^{\prime} \in \mathrm{A}} x \stackrel{a^{\prime}}{\longrightarrow} x^{\prime} \wedge \exists ! y \bullet\left(u^{\prime \prime} \stackrel{b}{\rightarrow} y \wedge x^{\prime} \rightarrow y\right) \Rightarrow \exists ! y \bullet\left(u^{\prime \prime} \stackrel{b}{\rightarrow} y \wedge x \rightarrow y\right) .
\end{aligned}
$$

The left-hand sides of the first and second implication are conjunctions of $u \stackrel{a}{\rightarrow} u^{\prime}$ and (an instance of) one of the first two conjuncts occurring in the right-hand side of $\rho\left(u^{\prime}, u^{\prime \prime}\right)$. The left-hand side of the third implication is a conjunction of the second conjunct occurring in the right-hand side of the first implication and 
(an instance of) the third conjunct occurring in the right-hand side of $\rho\left(u^{\prime}, u^{\prime \prime}\right)$. Hence, we also conclude that

$$
\forall u, u^{\prime}, u^{\prime \prime} \bullet\left(u \stackrel{a}{\rightarrow} u^{\prime} \wedge \rho\left(u^{\prime}, u^{\prime \prime}\right) \Rightarrow \rho\left(u, u^{\prime \prime}\right)\right) .
$$

Using the restricted subprocess induction schema, it follows from these conclusions that $u \stackrel{a}{\longrightarrow} u^{\prime} \Rightarrow \rho\left(u, u^{\prime}\right)$.

The following is a corollary of Theorem 12.1 .

Corollary 12.1 (RRS Implies RS). We have $\mathrm{BPA}_{\delta \mathrm{rr}}^{\mathrm{fo}}=\mathrm{RS}$.

Moreover, in the models of $\mathrm{BPA}_{\delta \mathrm{rr}}^{\mathrm{fo}}, \mathrm{R}$ holds if RR holds.

Theorem 12.2 (RR Implies R). We have $\mathrm{BPA}_{\delta \mathrm{rr}}^{\mathrm{fo}} \cup \mathrm{RR} \models \mathrm{R}$.

Proof. Suppose $\forall x^{\prime}, y^{\prime}, z^{\prime} \bullet\left(R\left(x^{\prime}, x^{\prime}\right) \wedge \bigwedge_{a \in \mathrm{A}}\left(x^{\prime} \stackrel{a}{\rightarrow} y^{\prime} \wedge R\left(y^{\prime}, z^{\prime}\right) \Rightarrow R\left(x^{\prime}, z^{\prime}\right)\right)\right)$. Then we must show that $\mathrm{BPA}_{\delta \mathrm{rr}}^{\mathrm{fo}} \cup \mathrm{RR}=x \rightarrow y \Rightarrow R(x, y)$. By Theorem 12.1, it is sufficient to show that $\forall u, u^{\prime} \bullet\left(\psi^{a, b}(x, u) \wedge u \stackrel{a}{\rightarrow} u^{\prime} \wedge u^{\prime} \stackrel{b}{\rightarrow} y \Rightarrow R(x, y)\right)$. This is done by induction on the number of steps, say $k$, required for $u \stackrel{a}{\rightarrow} u^{\prime}$. If $k=0$, then we immediately have $R(x, y)$. If $k=n+1$, then there exists a $u^{\prime \prime}$ such that $u \stackrel{a}{\longrightarrow} u^{\prime \prime}$ and $u^{\prime \prime} \stackrel{a}{\rightarrow} u^{\prime}$. It follows from $\psi^{a, b}(x, u)$, that there exists a unique $x^{\prime \prime}$ such that $x \stackrel{a^{\prime}}{\longrightarrow} x^{\prime \prime}$ for some action $a^{\prime}$ and $u^{\prime \prime} \stackrel{b}{\longrightarrow} x^{\prime \prime}$. By the induction hypothesis, $R\left(x^{\prime \prime}, y\right)$. From $x \stackrel{a^{\prime}}{\longrightarrow} x^{\prime \prime}$ and $R\left(x^{\prime \prime}, y\right)$, it follows that $R(x, y)$.

For each $\kappa \geq \aleph_{0}, \mathfrak{P}_{\kappa}^{\mathrm{rr}}$ is the expansion of $\mathfrak{P}_{\kappa}$ that additionally has for each

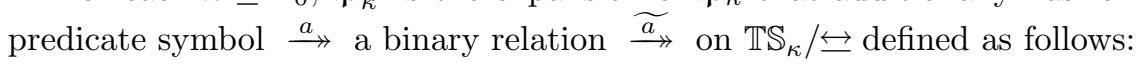

$$
\left[T_{1}\right] \stackrel{\widetilde{a}}{\rightarrow}\left[T_{2}\right] \text { iff } \exists T \in\left[T_{2}\right] \bullet T_{1} \stackrel{\widehat{a}}{\rightarrow} T,
$$

where $\underset{a_{\rightarrow}}{\rightarrow}$ is a binary relation on $\mathbb{T S}_{\kappa}$ which will be defined below. However, we first introduce an auxiliary notion. Let $T=\left(S, \rightarrow, \rightarrow \sqrt{ }, s^{0}\right)$ be a transition system. Then, for each $a \in \mathrm{A}$, the reachability by a-steps relation of $T$ is the smallest relation $\stackrel{a}{\rightarrow} \subseteq S \times S$ such that:

$-s \stackrel{a}{\rightarrow} s$

- if $s \stackrel{a}{\longrightarrow} s^{\prime}$ and $s^{\prime} \stackrel{a}{\longrightarrow} s^{\prime \prime}$, then $s \stackrel{a}{\longrightarrow} s^{\prime \prime}$.

We write $\operatorname{RS}_{a}(T)$ for $\left\{s \in S \mid s^{0} \stackrel{a}{\rightarrow} s\right\}$. Now the relation $\stackrel{\widehat{a}}{\rightarrow}$ on $\mathbb{T} \mathbb{S}_{\kappa}$ is defined as follows. Let $T_{1}, T_{2} \in \mathbb{T S} \mathbb{S}_{\kappa}$. Then

$$
T_{1} \stackrel{\widehat{a}}{\rightarrow} T_{2} \text { iff } \exists s \in \operatorname{RS}_{a}\left(T_{1}\right) \bullet\left(T_{1}\right)_{s}=T_{2} .
$$

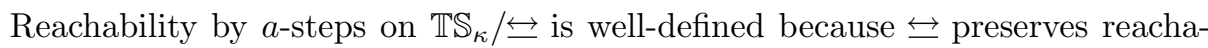
bility by $a$-steps on $\mathbb{T S}_{\kappa}$ up to $\leftrightarrows$.

The structures $\mathfrak{P}_{\kappa}^{\mathrm{rr}}$ are models of $\mathrm{BPA}_{\delta \mathrm{rr}}^{\mathrm{fo}}$.

Theorem 12.3 (Soundness of $\mathbf{B P A}_{\delta \mathrm{rr}}^{\mathrm{fo}}$ ). For all $\kappa \geq \aleph_{0}$, we have $\mathfrak{P}_{\kappa}^{\mathrm{rr}}=$ $\mathrm{BPA}_{\delta \mathrm{rr}}^{\mathrm{fo}}$. 
Table 9. Bar Induction Schema

$\bigwedge_{a \in \mathrm{A}} \psi(a) \wedge \forall x \bullet\left(\neg \infty(x) \Rightarrow\left(\forall y \bullet \bigwedge_{a \in \mathrm{A}}(x \stackrel{a}{\rightarrow} y \Rightarrow \psi(y)) \Rightarrow \psi(x)\right)\right) \Rightarrow$

$$
\forall x \bullet(\neg \infty(x) \Rightarrow \psi(x)) \quad \text { BAR }
$$

Proof. The soundness of the additional axioms for restricted reachability follows easily from the definition of the relation associated with each predicate symbol $\stackrel{a}{\longrightarrow}$ in $\mathfrak{P}_{\kappa}^{\mathrm{rr}}$.

It is unknown to us whether the restricted reachability predicates $\stackrel{a}{\rightarrow}$ are definable in terms of $\mathcal{L}\left(\mathrm{BPA}_{\delta}^{\text {fo }}\right)$ in $\mathrm{BPA}_{\delta}^{\text {fo }}$. In any case, the extension turns out to have great expressive power. Consider the following formula of $\mathcal{L}\left(\mathrm{BPA}_{\delta \mathrm{rr}}^{\text {fo }}\right)$ :

$$
\begin{aligned}
& \exists z \bullet(\forall u \bullet(z \stackrel{a}{\longrightarrow} u \Rightarrow \exists ! v \bullet u \stackrel{a}{\longrightarrow} v \wedge \\
& \left.\exists ! u^{\prime} \bullet u \stackrel{b}{\rightarrow} u^{\prime} \wedge \bigwedge_{a^{\prime} \in \mathrm{A}, a^{\prime} \neq a, b} \neg \exists w \bullet u \stackrel{a^{\prime}}{\longrightarrow} w\right) \wedge \\
& z \stackrel{b}{\rightarrow} x \wedge \\
& \forall u, v \bullet(z \stackrel{a}{\longrightarrow} u \wedge u \stackrel{a}{\longrightarrow} v \Rightarrow \\
& \left.\left.\exists u^{\prime}, v^{\prime} \bullet\left(u \stackrel{b}{\rightarrow} u^{\prime} \wedge v \stackrel{b}{\rightarrow} v^{\prime} \wedge \bigvee_{a^{\prime} \in \mathrm{A}} u^{\prime} \stackrel{a^{\prime}}{\longrightarrow} v^{\prime}\right)\right)\right),
\end{aligned}
$$

where $a$ and $b$ are different actions. We use $\infty(x)$ as an abbreviation of the above formula. Let $\mathfrak{A}$ be a model of $\mathrm{BPA}_{\delta \mathrm{rr}}^{\text {fo }}$ with domain $P$, and let $p \in P$. Then $\mathfrak{A}=\neg \infty(x)[p]$ only if $p$ has no infinite path in $\operatorname{TS}(\mathfrak{A}, p)$. If $\mathfrak{A}$ is one of the full bisimulation models $\mathfrak{P}_{\kappa}^{\text {rr }}$, "only if" can be replaced by "if and only if". There is no formula of $\mathcal{L}\left(\mathrm{BPA}_{\delta}^{\text {fo }}\right)$ with analogous properties.

The axiom schema BAR given in Table 9 can be used to prove properties of all processes that have no infinite path. BAR is an axiom schema in which $\psi$ stands for an arbitrary formula of $\mathcal{L}\left(\mathrm{BPA}_{\delta \mathrm{rr}}^{\text {fo }}\right)$ in which only the variable $x_{1}$ may have free occurrences. Axiom schema BAR is an induction schema, called the bar induction schema.

BAR is valid in the full bisimulation models $\mathfrak{P}_{\kappa}^{\text {rr }}$.

Theorem 12.4 (Soundness of BAR). For all $\kappa \geq \aleph_{0}$, we have $\mathfrak{P}_{\kappa}^{\text {rr }} \models$ BAR.

Proof. We define an ordinal function $\left\|_{-}\right\|$on the domain $\mathcal{P}$ of $\mathfrak{P}_{\kappa}^{\text {rr }}$ as follows:

- if $\left\{p^{\prime} \mid p \stackrel{\longrightarrow}{\rightrightarrows} p^{\prime}\right\}=\emptyset$, then $\|p\|=0$;

- if $\left\{p^{\prime} \mid p \underset{\rightarrow}{\dddot{\longrightarrow}} p^{\prime}\right\} \neq \emptyset$ and $\left\{\left\|p^{\prime}\right\| \mid p \underset{\rightarrow}{\rightarrow} p^{\prime}\right\}$ has a maximal element, then $\|p\|=\max \left\{\left\|p^{\prime}\right\| \mid p \underset{\rightarrow}{\rightarrow} p^{\prime}\right\}+1$

- if $\left\{p^{\prime} \mid p \stackrel{\leftrightarrow}{\rightarrow} p^{\prime}\right\} \neq \emptyset$ and $\left\{\left\|p^{\prime}\right\| \mid p \stackrel{\sim}{\rightarrow} p^{\prime}\right\}$ has no maximal element, then $\|p\|=\sup \left\{\left\|p^{\prime}\right\| \mid p \underset{\sim}{\dddot{\prime}} p^{\prime}\right\}$.

Because $p \stackrel{\widetilde{a}}{\longrightarrow} p^{\prime}$ implies $\left\|p^{\prime}\right\|<\|p\|$ if $p$ has no infinite path, it is easily proved by transfinite induction on $\|x\|$ that $\mathrm{BAR}$ is valid in $\mathfrak{P}_{\kappa}^{\mathrm{rr}}$. 


\section{The First-Order Theory ACP $\mathrm{Ao}^{\text {fo }}$}

In this section, we present $\mathrm{ACP}^{\text {fo }}$, a first-order extension of $\mathrm{ACP}$. Like in $\mathrm{BPA}^{\text {fo }}$, it is assumed that there is a fixed but arbitrary finite set of actions $\mathrm{A}$ with $\delta \notin \mathrm{A}$. We write $\mathrm{A}_{\delta}$ for $\mathrm{A} \cup\{\delta\}$. In $\mathrm{ACP}^{\text {fo }}$, it is further assumed that there is a fixed but arbitrary commutative and associative communication function $\mid: \mathrm{A}_{\delta} \times \mathrm{A}_{\delta} \rightarrow \mathrm{A}_{\delta}$ such that $\delta \mid a=\delta$ for all $a \in \mathrm{A}_{\delta}$.

The first-order theory $\mathrm{ACP}^{\text {fo }}$ is an extension of $\mathrm{BPA}_{\delta}^{\text {fo }}$. It has the nonlogical symbols of $\mathrm{BPA}_{\delta}^{\text {fo }}$ and in addition:

- the binary parallel composition operator $\|$;

- the binary left merge operator $\amalg$;

- the binary communication merge operator $\mid$;

- for each $H \subseteq \mathrm{A}$, the unary encapsulation operator $\partial_{H}$.

We use infix notation for the additional binary operators as well. The precedence conventions for the binary operators are now as follows. The operator $\cdot$ binds stronger than all other binary operators and the operator + binds weaker than all other binary operators.

The constants and operators of $\mathrm{ACP}^{\text {fo }}$ are the same as the constants and operators of ACP.

Let $t$ and $t^{\prime}$ be closed terms of $\mathcal{L}\left(\mathrm{ACP}^{\text {fo }}\right)$. Intuitively, the additional operators can be explained as follows:

- $t \| t^{\prime}$ behaves as the process that proceeds with $t$ and $t^{\prime}$ in parallel;

- $t \Perp t^{\prime}$ behaves the same as $t \| t^{\prime}$, except that it starts with performing an action of $t$;

- $t \mid t^{\prime}$ behaves the same as $t \| t^{\prime}$, except that it starts with performing an action of $t$ and an action of $t^{\prime}$ synchronously;

- $\partial_{H}(t)$ behaves the same as $t$, except that it does not perform actions in $H$.

The axioms of $\mathrm{ACP}^{\text {fo }}$ are the axioms of $\mathrm{BPA}_{\delta}^{\text {fo }}$ and the additional axioms given in Table 10. CM2-CM3, CM5-CM7, C1-C3 and D1-D2 are actually axiom schemas in which $a, b$ and $c$ stand for arbitrary constants of $\mathrm{ACP}^{\text {fo }}$ (i.e. $a, b, c \in$ $\mathrm{A}_{\delta}$ ). In D1-D4, $H$ stands for an arbitrary subset of A. So, D3 and D4 are axiom schemas as well.

Axioms A1-A7, CM1-CM9, C1-C3 and D1-D4 are the axioms of ACP. So $\mathrm{ACP}^{\text {fo }}$ imports the (equational) axioms of $\mathrm{ACP}$.

A well-known subtheory of ACP is PA, ACP without communication. Likewise, we have a subtheory of $\mathrm{ACP}^{\text {fo }}$, to wit $\mathrm{PA}^{\text {fo }}$. The first-order theory $\mathrm{PA}^{\text {fo }}$ is $\mathrm{ACP}^{\text {fo }}$ without the communication merge operator, without axioms CM5-CM9 and $\mathrm{C} 1-\mathrm{C} 3$, and with axiom CM1 replaced by $x \| y=x \llbracket y+y \llbracket x$ (M1). In other words, the possibility that actions are performed synchronously is not covered by $\mathrm{PA}^{\text {fo }}$.

To prove a statement for all closed terms of $\mathcal{L}\left(\mathrm{ACP}^{\text {fo }}\right)$, it is sufficient to prove it for all basic terms over $\mathrm{BPA}_{\delta}^{\text {fo }}$. The reason for this is that all closed terms of $\mathcal{L}\left(\mathrm{ACP}^{\text {fo }}\right)$ are derivably equal to a basic term over $\mathrm{BPA}_{\delta}^{\text {fo }}$. 
Table 10. Additional Axioms for $\operatorname{ACP}^{\text {fo }}\left(a, b, c \in \mathrm{A}_{\delta}\right)$

\begin{tabular}{|c|c|c|c|}
\hline$x \| y=x \llbracket y+y \llbracket x+x \mid y$ & CM1 & $a|b=b| a$ & $\mathrm{C} 1$ \\
\hline$a \llbracket x=a \cdot x$ & CM2 & $(a \mid b)|c=a|(b \mid c)$ & $\mathrm{C} 2$ \\
\hline$a \cdot x \llbracket y=a \cdot(x \| y)$ & CM3 & $\delta \mid a=\delta$ & $\mathrm{C} 3$ \\
\hline$(x+y) \llbracket z=x \llbracket z+y \llbracket z$ & CM4 & & \\
\hline$a \cdot x \mid b=(a \mid b) \cdot x$ & CM5 & $\partial_{H}(a)=a$ & $\mathrm{D}$ \\
\hline$a \mid b \cdot x=(a \mid b) \cdot x$ & CM6 & $\partial_{H}(a)=\delta$ & $\mathrm{D} 2$ \\
\hline$a \cdot x \mid b \cdot y=(a \mid b) \cdot(x \| y)$ & CM7 & $\partial_{H}(x+y)=\partial_{H}(x)+\partial_{H}(y)$ & $\mathrm{D}$ \\
\hline$(x+y)|z=x| z+y \mid z$ & CM8 & $\partial_{H}(x \cdot y)=\partial_{H}(x) \cdot \partial_{H}(y)$ & $\mathrm{D}$ \\
\hline$x|(y+z)=x| y+x \mid z$ & CM9 & & \\
\hline
\end{tabular}

Proposition 13.1 (Elimination). For all closed terms $t$ of $\mathcal{L}\left(\mathrm{ACP}^{\mathrm{fo}}\right)$ there exists a basic term $t^{\prime}$ such that $\mathrm{ACP}^{\text {fo }} \vdash t=t^{\prime}$.

Proof. This follows immediately from the elimination property for ACP: the closed terms of $\mathcal{L}\left(\mathrm{ACP}^{\text {fo }}\right)$ are the same as the closed terms of $\mathcal{L}(\mathrm{ACP})$, and the equational axioms of $\mathrm{ACP}^{\text {fo }}$ are the same as the axioms of $\mathrm{ACP}$.

For closed equations, $\mathrm{ACP}^{\text {fo }}$ is a complete theory.

Theorem 13.1 (Complete Theory for Closed Equations). For all closed terms $t_{1}, t_{2}$ of $\mathcal{L}\left(\mathrm{ACP}^{\text {fo }}\right)$, we have either $\mathrm{ACP}^{\text {fo }} \vdash t_{1}=t_{2}$ or $\mathrm{ACP}^{\text {fo }} \vdash \neg t_{1}=t_{2}$, but not both.

Proof. This follows immediately from Proposition 13.1 and Theorem 2.1.

We have not yet investigated the decidability of $\mathrm{ACP}^{\text {fo }}$, but it is to be expected that it is an undecidable theory. By adaptation of the proof of a similar theorem from [11], we can easily establish the undecidability of $\mathrm{ACP}^{\text {fo }} \cup \mathrm{AIP}$. Here, and in Theorem 13.2, we abuse the name AIP for $\left\{\mathrm{OBS}_{n} \mid n \geq 0\right\} \cup$ $\{\mathrm{OBS}, \mathrm{AIP}\}$.

Theorem 13.2 (Undecidability). $\mathrm{ACP}^{\mathrm{fo}} \cup \mathrm{AIP}$ is an undecidable theory.

Proof. We consider a register machine with three registers, numbered 1, 2 and 3. A program for the register machine is a finite sequence $I_{1}, \ldots, I_{k}$ of instructions of the following form:

- $\left(\operatorname{add}_{i}, j\right)$ : add 1 to the contents of register $i$ and go to instruction $j$;

- $\left(\operatorname{sub}_{i}, j\right)$ : if the contents of register $i$ equals 0 , then go to instruction $j$, otherwise subtract 1 from the contents of register $i$ and go to instruction $j$;

- $\left(\right.$ zero $\left._{i}, j, j^{\prime}\right)$ : if the contents of register $i$ equals 0 , then go to instruction $j$, otherwise go to instruction $j^{\prime}$;

- halt: halt; 
where $i \in\{1,2,3\}$ and $j, j^{\prime} \in\{1, \ldots, k\}$.

Let $K$ be a recursively enumerable but not recursive subset of $\mathbb{N}$, and let $n \in \mathbb{N}$. Then there exists a program for this register machine such that, if the registers are initialized to $n, 0$ and 0 , the program halts iff $n \in K$ (see e.g. [25]). Let $P=I_{1}, \ldots, I_{l}$ be this program. We will show that $P$ can be represented in $\mathrm{ACP}^{\text {fo }} \cup \mathrm{AIP}$.

Let $A=\left\{a_{i}, s_{i}, z_{i} \mid 1 \leq i \leq 3\right\}$ and $\bar{A}=\left\{\bar{a}_{i}, \bar{s}_{i}, \bar{z}_{i} \mid 1 \leq i \leq 3\right\}$. We fix the set of actions $\mathrm{A}$ and the communication function $\mid$ as follows: $\mathrm{A}=A \cup \bar{A} \cup\{t, h\}$; and $a \mid b=t$ if either $a \in A, b \in \bar{A}$ and $\bar{a}=b$, or $a \in \bar{A}, b \in A$ and $a=\bar{b}$, and $a \mid b=\delta$ otherwise.

Let $E$ be the guarded recursive specification that consists of the following equations:

$$
\begin{array}{ll}
R_{i}=\bar{z}_{i} \cdot R_{i}+\bar{a}_{i} \cdot R_{i}^{\prime} \cdot R_{i} & \text { for } i \in\{1,2,3\}, \\
R_{i}^{\prime}=\bar{s}_{i}+\bar{a}_{i} \cdot R_{i}^{\prime} \cdot R_{i}^{\prime} & \text { for } i \in\{1,2,3\}, \\
X_{j}=\llbracket I_{j} \rrbracket & \text { for } j \in\{1, \ldots, l\}, \\
T=t \cdot T, &
\end{array}
$$

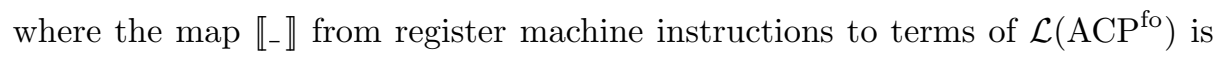
defined as follows:

$$
\begin{array}{ll}
\llbracket\left(\operatorname{add}_{i}, j\right) \rrbracket & =a_{i} \cdot X_{j}, \\
\llbracket\left(\operatorname{sub}_{i}, j\right) \rrbracket & =\left(z_{i}+s_{i}\right) \cdot X_{j}, \\
\llbracket\left(\operatorname{zero}_{i}, j, j^{\prime}\right) \rrbracket & =z_{i} \cdot X_{j}+s_{i} \cdot a_{i} \cdot X_{j^{\prime}}, \\
\llbracket \text { halt } \rrbracket & =h .
\end{array}
$$

We introduce for $m \geq 0$ the abbreviation $R_{i}(m)$ defined by $R_{i}(0)=R_{i}$ and $R_{i}(m+1)=R_{i}^{\prime} \cdot R_{i}(m)$. Note that $R_{i}(m)$ represents register $i$ in the state where its contents is $m$.

It is easy to see that $P$ does not halt iff

$$
\mathrm{ACP}^{\mathrm{fo}} \cup \mathrm{AIP} \vdash E \Rightarrow \partial_{H}\left(X_{1}\left\|R_{1}(n)\right\| R_{2}(0) \| R_{3}(0)\right)=T,
$$

where $H=A \cup \bar{A}$. Therefore, the problem whether $n \notin K$ is one to one reducible to the problem whether a given formula of $\mathcal{L}\left(\mathrm{ACP}^{\mathrm{fo}} \cup \mathrm{AIP}\right)$ is derivable. Because the former problem is undecidable, we conclude that the latter problem is undecidable as well. This shows that $\mathrm{ACP}^{\mathrm{fo}} \cup \mathrm{AIP}$ is an undecidable theory.

In this section, $\mathrm{BPA}_{\delta}^{\text {fo }}$ has been extended to $\mathrm{ACP}^{\mathrm{fo}}$. $\mathrm{BPA}_{\delta \mathrm{rr}}^{\text {fo }}$ can be extended with the same nonlogical symbols and axioms as $\mathrm{BPA}_{\delta}^{\mathrm{fo}}$, resulting in $\mathrm{ACP}_{\mathrm{rr}}^{\mathrm{fo}}$. 


\section{Full Bisimulation Models of $\mathrm{ACP}^{\text {fo }}$}

In this section, we expand the full bisimulation models of $\mathrm{BPA}_{\delta}^{\text {fo }}$ to $\mathrm{ACP}^{\text {fo }}$. We will use the abbreviation $s \stackrel{a}{\rightarrow} s^{\prime} \chi s^{\prime \prime}$ for $s \stackrel{a}{\longrightarrow} s^{\prime} \vee\left(s \stackrel{a}{\longrightarrow} \sqrt{ } \wedge s^{\prime}=s^{\prime \prime}\right)$.

First of all, we associate with each additional operator $f$ of $\mathrm{ACP}^{\text {fo }}$ an operation $\widehat{f}$ on $\mathbb{T} \mathbb{S}_{\kappa}$ as follows.

- Let $T_{i}=\left(S_{i}, \rightarrow_{i}, \rightarrow{ }, s_{i}^{0}\right) \in \mathbb{T} \mathbb{S}_{\kappa}$ for $i=1,2$, and $s^{\vee} \notin S_{1} \cup S_{2}$. Then

$$
T_{1} \Uparrow T_{2}=\left(S, \rightarrow, \rightarrow \sqrt{ }, s^{0}\right)
$$

where

$$
\begin{aligned}
& S=\left(\left(S_{1} \cup\left\{s^{\vee}\right\}\right) \times\left(S_{2} \cup\left\{s^{\vee}\right\}\right)\right) \backslash\left\{\left(s^{\vee}, s^{\vee}\right)\right\}, \\
& s^{0}=\left(s_{1}^{0}, s_{2}^{0}\right)
\end{aligned}
$$

and for every $a \in \mathrm{A}$ :

$$
\begin{aligned}
& \stackrel{a}{\longrightarrow}=\left\{\left(\left(s_{1}, s_{2}\right),\left(s_{1}^{\prime}, s_{2}\right)\right) \mid\left(s_{1}^{\prime}, s_{2}\right) \in S \wedge s_{1} \stackrel{a}{\longrightarrow} 1 s_{1}^{\prime}\left\langle s^{\vee}\right\}\right. \\
& \cup\left\{\left(\left(s_{1}, s_{2}\right),\left(s_{1}, s_{2}^{\prime}\right)\right) \mid\left(s_{1}, s_{2}^{\prime}\right) \in S \wedge s_{2} \stackrel{a}{\longrightarrow}_{2} s_{2}^{\prime}\left\langle s^{\vee}\right\}\right. \\
& \cup\left\{\left(\left(s_{1}, s_{2}\right),\left(s_{1}^{\prime}, s_{2}^{\prime}\right)\right) \mid\left(s_{1}^{\prime}, s_{2}^{\prime}\right) \in S \wedge\right. \\
& \left.\bigvee_{a^{\prime}, b^{\prime} \in \mathrm{A}}\left(s_{1} \stackrel{a^{\prime}}{\longrightarrow} 1 s_{1}^{\prime} \prec s^{\vee} \wedge s_{2}{\stackrel{b^{\prime}}{\longrightarrow}}_{2} s_{2}^{\prime}\left\langle s^{\vee} \wedge a^{\prime}\right| b^{\prime}=a\right)\right\}, \\
& \stackrel{a}{\rightarrow} \sqrt{ }=\left\{\left(s_{1}, s^{\sqrt{ }}\right) \mid s_{1} \stackrel{a}{\longrightarrow} \sqrt{ }_{1}\right\} \cup\left\{\left(s^{\vee}, s_{2}\right) \mid s_{2} \stackrel{a}{\longrightarrow} \sqrt{ }_{2}\right\} \\
& \cup\left\{\left(s_{1}, s_{2}\right) \mid \bigvee_{a^{\prime}, b^{\prime} \in \mathrm{A}}\left(s_{1} \stackrel{a^{\prime}}{\longrightarrow} \sqrt{ }_{1} \wedge s_{2} \stackrel{b^{\prime}}{\longrightarrow} \sqrt{ }_{2} \wedge a^{\prime} \mid b^{\prime}=a\right)\right\} .
\end{aligned}
$$

- Let $T_{i}=\left(S_{i}, \rightarrow_{i}, \rightarrow{ } i, s_{i}^{0}\right) \in \mathbb{T} \mathbb{S}_{\kappa}$ for $i=1,2$, and $s^{\vee} \notin S_{1} \cup S_{2}$. Suppose that $T_{1} \Uparrow T_{2}=\left(S, \rightarrow, \rightarrow \sqrt{ }, s^{0}\right)$ with $S=\left(\left(S_{1} \cup\left\{s^{\vee}\right\}\right) \times\left(S_{2} \cup\left\{s^{\vee}\right\}\right)\right) \backslash\left\{\left(s^{\vee}, s^{\vee}\right)\right\}$ and $s^{0 \prime} \notin S$. Then

$$
T_{1} \Uparrow T_{2}=\Gamma\left(S^{\prime}, \rightarrow^{\prime}, \rightarrow \sqrt{ }, s^{0 \prime}\right),
$$

where

$$
S^{\prime}=\left\{s^{0 \prime}\right\} \cup S,
$$

and for every $a \in \mathrm{A}$ :

$$
\stackrel{a}{\longrightarrow}^{\prime}=\left\{\left(s^{0 \prime},\left(s, s_{2}^{0}\right)\right) \mid s_{1}^{0} \stackrel{a}{\longrightarrow}_{1} s\left\{s^{\vee}\right\} \cup \stackrel{a}{\longrightarrow} .\right.
$$

- Let $T_{i}=\left(S_{i}, \rightarrow_{i}, \rightarrow \sqrt{ }_{i}, s_{i}^{0}\right) \in \mathbb{T} \mathbb{S}_{\kappa}$ for $i=1,2$, and $s^{\vee} \notin S_{1} \cup S_{2}$. Suppose that $T_{1} \Uparrow T_{2}=\left(S, \rightarrow, \rightarrow \sqrt{ }, s^{0}\right)$ with $S=\left(\left(S_{1} \cup\left\{s^{\vee}\right\}\right) \times\left(S_{2} \cup\left\{s^{\vee}\right\}\right)\right) \backslash\left\{\left(s^{\vee}, s^{\vee}\right)\right\}$ and $s^{0 \prime} \notin S$. Then

$$
T_{1} \widehat{\uparrow} T_{2}=\Gamma\left(S^{\prime}, \rightarrow^{\prime}, \rightarrow \sqrt{ }^{\prime}, s^{0 \prime}\right)
$$

where

$$
S^{\prime}=\left\{s^{0 \prime}\right\} \cup S,
$$


and for every $a \in \mathrm{A}$ :

$$
\begin{aligned}
& \stackrel{a}{\longrightarrow}=\left\{\left(s^{0 \prime},\left(s_{1}, s_{2}\right)\right) \mid\left(s_{1}, s_{2}\right) \in S \wedge\right. \\
& \bigvee_{a^{\prime}, b^{\prime} \in \mathrm{A}}\left(s_{1}^{0} \stackrel{a^{\prime}}{\longrightarrow} s_{1}\left\langle s^{\vee} \wedge s_{2}^{0} \stackrel{b}{\longrightarrow}_{2} s_{2}\left\{s^{\vee} \wedge a^{\prime} \mid b^{\prime}=a\right)\right\} \cup \stackrel{a}{\longrightarrow},\right. \\
& \stackrel{a}{\longrightarrow} \sqrt{ }^{\prime}=\left\{s^{0 \prime} \mid \bigvee_{a^{\prime}, b^{\prime} \in \mathrm{A}}\left(s_{1}^{0} \stackrel{a^{\prime}}{\longrightarrow} \sqrt{ }_{1} \wedge s_{2}^{0} \stackrel{b^{\prime}}{\longrightarrow} \sqrt{ }_{2} \wedge a^{\prime} \mid b^{\prime}=a\right)\right\} \cup \stackrel{a}{\longrightarrow} \sqrt{ } .
\end{aligned}
$$

- Let $T=\left(S, \rightarrow, \rightarrow \sqrt{ }, s^{0}\right) \in \mathbb{T} \mathbb{S}_{\kappa}$. Then

$$
\widehat{\partial H}(T)=\Gamma\left(S, \rightarrow^{\prime}, \rightarrow \sqrt{ }^{\prime}, s^{0}\right),
$$

where for every $a \notin H$ :

$$
\begin{aligned}
& \stackrel{a}{\longrightarrow}=\stackrel{a}{\longrightarrow}, \\
& \stackrel{a}{\longrightarrow} \sqrt{ }^{\prime}=\stackrel{a}{\longrightarrow} \sqrt{ },
\end{aligned}
$$

and for every $a \in H$ :

$$
\begin{aligned}
& \stackrel{a}{\longrightarrow}=\emptyset, \\
& \stackrel{a}{\longrightarrow} \sqrt{ }^{\prime}=\emptyset .
\end{aligned}
$$

We can easily show that bisimilarity is a congruence with respect to parallel composition, left merge, communication merge and encapsulation.

Proposition 14.1 (Congruence). For all $T_{1}, T_{2}, T_{1}^{\prime}, T_{2}^{\prime} \in \mathbb{T S}_{\kappa}\left(\kappa \geq \aleph_{0}\right), T_{1} \leftrightarrows$

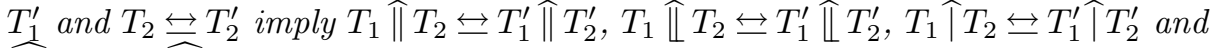

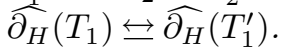

Proof. Let $R_{1}$ and $R_{2}$ be bisimulations witnessing $T_{1} \leftrightarrows T_{1}^{\prime}$ and $T_{2} \leftrightarrows T_{2}^{\prime}$, respectively. Then we construct relations $R_{\Uparrow}, R_{\widehat{\Perp}}, R_{\uparrow}$ and $R_{\widehat{\partial_{H}}}$ as follows:

$-R_{\Uparrow}=\left\{\left(\left(s_{1}, s_{2}\right),\left(s_{1}^{\prime}, s_{2}^{\prime}\right)\right) \mid\left(s_{1}, s_{1}^{\prime}\right) \in R_{1},\left(s_{2}, s_{2}^{\prime}\right) \in R_{2}\right\}$

$-R_{\widehat{\Perp}}=\left(\left\{\left(s^{0}, s^{0 \prime}\right)\right\} \cup R_{\Uparrow}\right) \cap\left(S \times S^{\prime}\right)$, where $S$ and $S^{\prime}$ are the sets of states of $T_{1} \Uparrow T_{2}$ and $T_{1}^{\prime} \Uparrow T_{2}^{\prime}$, respectively, and $s^{0}$ and $s^{0 \prime}$ are the initial states of $T_{1} \Uparrow T_{2}$ and $T_{1}^{\prime} \Uparrow T_{2}^{\prime}$, respectively;

$-R_{\uparrow}=\left(\left\{\left(s^{0}, s^{0 \prime}\right)\right\} \cup R_{\Uparrow}\right) \cap\left(S \times S^{\prime}\right)$, where $S$ and $S^{\prime}$ are the sets of states of

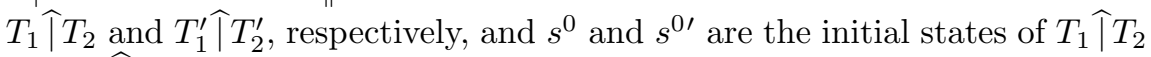
and $T_{1}^{\prime} \uparrow T_{2}^{\prime}$, respectively;

$-R_{\widehat{\partial_{H}}}=R_{1} \cap\left(S \times S^{\prime}\right)$, where $S$ and $S^{\prime}$ are the sets of states of $\widehat{\partial_{H}}\left(T_{1}\right)$ and $\widehat{\partial_{H}}\left(T_{1}^{\prime}\right)$, respectively.

Given the definitions of parallel composition, left merge, communication merge and encapsulation, it is easy to see that $R_{\widehat{\|}}, R_{\widehat{\Perp}}, R_{\Uparrow}$ and $R_{\widehat{\partial_{H}}}$ are bisimulations

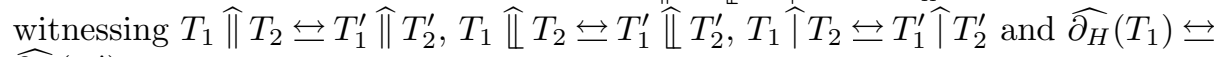
$\widehat{\partial_{H}}\left(T_{1}^{\prime}\right)$, respectively. 
The full bisimulation models $\mathfrak{P}_{\kappa}^{\prime}$ of $\mathrm{ACP}^{\text {fo }}$, one for each $\kappa \geq \aleph_{0}$, are the expansions of the full bisimulation models $\mathfrak{P}_{\kappa}$ of $\mathrm{BPA}_{\delta}^{\text {fo }}$ with an $n$-ary operation

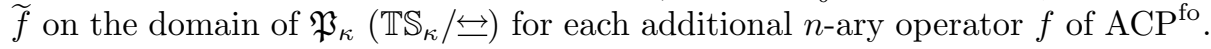
Those additional operations are defined as follows:

$$
\begin{aligned}
& {\left[T_{1}\right] \widetilde{\|}\left[T_{2}\right]=\left[T_{1} \widehat{\|} T_{2}\right],} \\
& {\left[T_{1}\right] \widetilde{\mathbb{L}}\left[T_{2}\right]=\left[T_{1} \widehat{\amalg} T_{2}\right] \text {, }} \\
& {\left[T_{1}\right] \widetilde{\lceil}\left[T_{2}\right]=\left[T_{1} \uparrow T_{2}\right],} \\
& \widetilde{\partial_{H}}\left(\left[T_{1}\right]\right)=\left[\widehat{\partial_{H}}\left(T_{1}\right)\right] \text {. }
\end{aligned}
$$

Parallel composition, left merge, communication merge and encapsulation on

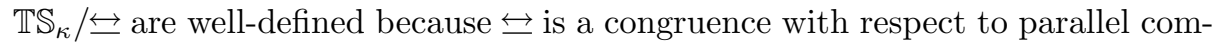
position, left merge, communication merge and encapsulation on $\mathbb{T} \mathbb{S}_{\kappa}$.

The structures $\mathfrak{P}_{\kappa}^{\prime}$ are models of $\mathrm{ACP}^{\text {fo }}$.

Theorem 14.1 (Soundness of $\mathbf{A C P}^{\text {fo }}$ ). For all $\kappa \geq \aleph_{0}$, we have $\mathfrak{P}_{\kappa}^{\prime}=$ $\mathrm{ACP}^{\text {fo }}$.

Proof. The soundness of all additional axioms follows easily from the definition of the domain $\mathcal{P}$ of $\mathfrak{P}_{\kappa}^{\prime}$ and the definitions of the elements of $\mathcal{P}$, the operations on $\mathcal{P}$ and the relations on $\mathcal{P}$ associated with the constants, operators and predicate symbols of $\mathrm{ACP}^{\text {fo }}$ in $\mathfrak{P}_{\kappa}^{\prime}$.

It is easy to see that Theorems $5.2,5.4$ and 5.5 go through for $\mathfrak{P}_{\kappa}^{\prime}$.

In this section, the full bisimulation models $\mathfrak{P}_{\kappa}$ of $\mathrm{BPA}_{\delta}^{\text {fo }}$ have been expanded to obtain the full bisimulation models $\mathfrak{P}_{\kappa}^{\prime}$ of $\mathrm{ACP}^{\text {fo }}$. The full bisimulation models $\mathfrak{P}_{\kappa}^{\text {rr }}$ of $\mathrm{BPA}_{\delta \mathrm{rr}}^{\mathrm{fo}}$ can be expanded in the same way to obtain the full bisimulation models $\mathfrak{P}_{\kappa}^{\mathrm{rr} \prime}$ of $\mathrm{ACP}_{\mathrm{rr}}^{\mathrm{fo}}$.

Henceforth, we will loosely write $\mathfrak{P}_{\kappa}$ for $\mathfrak{P}_{\kappa}^{\prime}$, and $\mathfrak{P}_{\kappa}^{\text {rr }}$ for $\mathfrak{P}_{\kappa}^{\text {rr’ }}$. It is always made sure that no confusion between the original model and its expansion may arise.

\section{Interpretation of One Theory in Another}

Let $T$ be a first-order theory with non-logical symbols $\Sigma$. Then we say that $\Sigma$ is the signature of $T$. We write $\Sigma(T)$ for the signature of $T$.

Let $T$ and $T^{\prime}$ be first-order theories, and $\mathfrak{d} \notin \Sigma(T) \cup \Sigma\left(T^{\prime}\right)$. Then an interpretation $\Theta$ of $T$ in $T^{\prime}$ is a family of formulas that consists of the following:

- an explicit definition $\Theta_{\mathfrak{d}}$ of a unary predicate $\mathfrak{d}$ in terms of $\mathcal{L}\left(T^{\prime}\right)$;

- for each $\sigma \in \Sigma(T) \backslash \Sigma\left(T^{\prime}\right)$, an explicit definition $\Theta_{\sigma}$ in terms of $\mathcal{L}\left(T^{\prime}\right)$;

such that the following holds for $T^{\prime \prime}=T^{\prime} \cup\left\{\Theta_{\sigma} \mid \sigma \in\left(\Sigma(T) \backslash \Sigma\left(T^{\prime}\right)\right) \cup\{\mathfrak{d}\}\right\}$ : 


$$
\begin{aligned}
& T^{\prime \prime} \vdash \exists x \bullet \mathfrak{d}(x), \\
& T^{\prime \prime} \vdash \mathfrak{d}\left(x_{1}\right) \wedge \ldots \wedge \mathfrak{d}\left(x_{n}\right) \Rightarrow \mathfrak{d}\left(f\left(x_{1}, \ldots, x_{n}\right)\right)
\end{aligned}
$$

for each $n$-ary operator $f \in \Sigma(T)$,

$$
T^{\prime \prime} \vdash \phi^{*}
$$

for each axiom $\phi$ of $T$,

where $\phi^{*}$ is the formula obtained from $\phi$ by first taking the universal closure of $\phi$ and then replacing each subformula $\forall x \bullet \phi^{\prime}$ by $\forall x \bullet\left(\mathfrak{d}(x) \Rightarrow \phi^{\prime}\right)$ and each subformula $\exists x \bullet \phi^{\prime}$ by $\exists x \bullet\left(\mathfrak{d}(x) \wedge \phi^{\prime}\right)$.

This notion of interpretation of one theory in another is more general than the corresponding notion from [28], but in line with the notion of interpretability of one theory in another from [28]. It is less general than the corresponding notion in [24]. In the terminology of [24], an interpretation as defined here is an injective one-dimensional interpretation. We believe that higher dimensional interpretations are irrelevant to the case where theories about processes are considered. So long as we only consider bisimilarity as the intended notion of identity, non-injective interpretations are irrelevant as well. Note that the last condition in the definition given above can be replaced by

$$
T \vdash \phi \text { implies } T^{\prime \prime} \vdash \phi^{*} \text { for each formula } \phi \text { of } \mathcal{L}(T) \text {. }
$$

The following is an important property of interpretations. For each interpretation $\Theta$ of a theory $T$ in a theory $T^{\prime}, T^{\prime \prime}=T^{\prime} \cup\left\{\Theta_{\sigma} \mid \sigma \in\left(\Sigma(T) \backslash \Sigma\left(T^{\prime}\right)\right) \cup\{\mathfrak{d}\}\right\}$ is a definitional extension of $T^{\prime}$. This means that, for each model $\mathfrak{A}^{\prime}$ of $T^{\prime}$, there is a unique expansion of $\mathfrak{A}^{\prime}$ that is a model of $T^{\prime \prime}$.

Let $\Theta$ be an interpretation of theory $T$ in theory $T^{\prime}$, and let $T^{\prime \prime}=T^{\prime} \cup\left\{\Theta_{\sigma} \mid\right.$ $\left.\sigma \in\left(\Sigma(T) \backslash \Sigma\left(T^{\prime}\right)\right) \cup\{\mathfrak{d}\}\right\}$. Suppose that $\mathfrak{A}^{\prime}$ is a model of $T^{\prime}$. Then a model $\mathfrak{A}$ of $T$ can be obtained from $\mathfrak{A}^{\prime}$ in the following steps:

1. take the unique expansion $\mathfrak{A}^{\prime \prime}$ of $\mathfrak{A}^{\prime}$ such that $\mathfrak{A}^{\prime \prime}$ is a model of $T^{\prime \prime}$;

2. take the restriction $\left.\mathfrak{A}^{\prime \prime}\right|_{\Sigma(T) \cup\{\mathfrak{d}\}}$ of $\mathfrak{A}^{\prime \prime}$ to $\Sigma(T) \cup\{\mathfrak{d}\}$;

3. take the unique substructure $\mathfrak{A}^{*}$ of $\left.\mathfrak{A}^{\prime \prime}\right|_{\Sigma(T) \cup\{\mathfrak{o}\}}$ such that $\mathfrak{A}^{*} \models \forall x \bullet \mathfrak{d}(x)$;

4. take the restriction $\mathfrak{A}=\left.\mathfrak{A}^{*}\right|_{\Sigma(T)}$ of $\mathfrak{A}^{*}$ to $\Sigma(T)$.

The most simple example of this construction is the following: The interpretation of BPA in $\mathrm{BPA}_{\delta}^{\text {fo }}$ consists only of the explicit definition $\mathfrak{d}(x) \Leftrightarrow \neg x \rightarrow \delta$. That is, $\mathfrak{d}$ is in this case just another symbol for the deadlock freedom predicate. If we apply the construction described above to one of the full bisimulation models of $\mathrm{BPA}_{\delta}^{\text {fo }}$, then we obtain one of the main models of BPA.

$\mathrm{MPA}_{\delta}$, Minimal Process Algebra with deadlock, provides another simple example. $\mathrm{MPA}_{\delta}$, introduced in [21], differs from $\mathrm{BPA}_{\delta}$ by having a unary action prefixing operator $a$. for each $a \in \mathrm{A}$ instead of the binary sequential composition operator of $\mathrm{BPA}_{\delta} \cdot{ }^{3}$ The axioms of $\mathrm{MPA}_{\delta}$ are axioms A1, A2, A3 and A6 from

\footnotetext{
${ }^{3}$ For action prefixing and sequential composition different kinds of dot, viz. the low dot and the centered dot, are used. In $\mathrm{MPA}_{\delta}$, we have action prefixing without variable binding. In [2], the semicolon is used for action prefixing with variable binding.
} 
Table 1. The interpretation of $\mathrm{MPA}_{\delta}$ in $\mathrm{BPA}_{\delta}^{\text {fo }}$ consists of the explicit definition $\mathfrak{d}(x) \Leftrightarrow \bigwedge_{a \in \mathrm{A}} \neg x \rightarrow a$ and an explicit definition $a \cdot x=y \Leftrightarrow a \cdot x=y$ for each $a \in A$. If we apply the construction described above to one of the full bisimulation models of $\mathrm{BPA}_{\delta}^{\text {fo }}$, then we obtain one of the main models of $\mathrm{MPA}_{\delta}$.

\section{Interpretation of $\mathrm{ACP}^{\text {fo }}$ in $\mathrm{BPA}_{\delta \mathrm{rr}}^{\text {fo }}$}

In this section, we consider the interpretation of $\mathrm{ACP}^{\mathrm{fo}}$ in $\mathrm{BPA}_{\delta \mathrm{rr}}^{\mathrm{fo}}$. This interpretation consists of explicit definitions of the predicate symbol $\mathfrak{d}$ and the operators $\|, \downarrow, \mid$ and $\partial_{H}$ (one for each $H \subseteq \mathrm{A}$ ). The explicit definition of $\mathfrak{d}$ is simply $\mathfrak{d}(x) \Leftrightarrow x=x$. The explicit definitions of the operators are quite unusual in the sense that they involve an auxiliary process $(u)$ that is used to represent a bisimulation.

First, we consider the explicit definition of the parallel composition operator. We begin by introducing the abbreviation $\mathrm{P}_{\|}^{\prime}(x, y, z, u)$, which enables us to formulate the explicit definition of $\|$ as $x \| y=z \Leftrightarrow \exists u \bullet \mathrm{P}_{\|}^{\prime}(x, y, z, u)$. We fix different actions $i, l, r$ and $m$. We use $\mathrm{P}_{\|}^{\prime}(x, y, z, u)$ as an abbreviation of the following formula of $\mathcal{L}\left(\mathrm{BPA}_{\delta \mathrm{rr}}^{\text {fo }}\right)$ :

$$
\begin{aligned}
& \phi_{1}(x, y, z, u) \wedge \phi_{2}(x, y, z, u) \wedge \phi_{3}(u) \wedge \phi_{4}(u) \wedge \phi_{5}(u) \wedge \\
& \phi_{6}(u) \wedge \phi_{7}(u) \wedge \phi_{8}(u) \wedge \phi_{9}(u) \wedge \phi_{10}(u) \wedge \phi_{11}(u) ;
\end{aligned}
$$

where:

$\phi_{1}(x, y, z, u)$ is the formula

$$
\begin{aligned}
& \forall u^{\prime} \bullet\left(u \stackrel{i}{\rightarrow} u^{\prime} \Rightarrow\right. \\
& \exists ! x^{\prime}, y^{\prime}, z^{\prime} \bullet\left(x \rightarrow x^{\prime}\right. \wedge y \rightarrow y^{\prime} \wedge z \rightarrow z^{\prime} \Rightarrow \\
& u^{\prime}\left.\stackrel{l}{\rightarrow} x^{\prime} \wedge u^{\prime} \stackrel{r}{\rightarrow} y^{\prime} \wedge u^{\prime} \stackrel{m}{\longrightarrow} z^{\prime}\right) \wedge \\
&\left.\bigwedge_{a^{\prime} \in \mathrm{A}, a^{\prime} \neq i, l, r, m} \neg \exists v^{\prime} \bullet u^{\prime} \stackrel{a^{\prime}}{\longrightarrow} v^{\prime}\right),
\end{aligned}
$$

$\phi_{2}(x, y, z, u)$ is the formula

$$
u \stackrel{l}{\rightarrow} x \wedge u \stackrel{r}{\longrightarrow} y \wedge u \stackrel{m}{\longrightarrow} z,
$$

$\phi_{3}(u)$ is the formula

$$
\begin{aligned}
& \forall x^{\prime}, y^{\prime}, z^{\prime}, u^{\prime}, x^{\prime \prime} \bullet \\
& \bigwedge_{a^{\prime} \in \mathrm{A}}\left(u \stackrel{i}{\rightarrow} u^{\prime \prime}, z^{\prime \prime} \wedge u^{\prime} \stackrel{l}{\rightarrow} x^{\prime} \wedge u^{\prime} \stackrel{r}{\rightarrow} y^{\prime} \wedge u^{\prime} \stackrel{m}{\longrightarrow} z^{\prime} \wedge x^{\prime} \stackrel{a^{\prime}}{\rightarrow} x^{\prime \prime} \Rightarrow\right. \\
& \left.\left(u^{\prime} \stackrel{i}{\rightarrow} u^{\prime \prime} \wedge u^{\prime \prime} \stackrel{l}{\rightarrow} x^{\prime \prime} \wedge u^{\prime \prime} \stackrel{r}{\rightarrow} y^{\prime} \wedge u^{\prime \prime} \stackrel{m}{\longrightarrow} z^{\prime \prime} \wedge z^{\prime} \stackrel{a^{\prime}}{\longrightarrow} z^{\prime \prime}\right)\right),
\end{aligned}
$$


$\phi_{4}(u)$ is the formula

$$
\begin{aligned}
& \forall x^{\prime}, y^{\prime}, z^{\prime}, u^{\prime}, y^{\prime \prime} \bullet \\
& \bigwedge_{a^{\prime} \in \mathrm{A}}\left(u \stackrel{i}{\rightarrow} u^{\prime \prime}, z^{\prime \prime} \bullet u^{\prime} \stackrel{l}{\rightarrow} x^{\prime} \wedge u^{\prime} \stackrel{r}{\rightarrow} y^{\prime} \wedge u^{\prime} \stackrel{m}{\longrightarrow} z^{\prime} \wedge y^{\prime} \stackrel{a^{\prime}}{\rightarrow} y^{\prime \prime} \Rightarrow\right. \\
& \left.\quad\left(u^{\prime} \stackrel{i}{\rightarrow} u^{\prime \prime} \wedge u^{\prime \prime} \stackrel{l}{\rightarrow} x^{\prime} \wedge u^{\prime \prime} \stackrel{r}{\rightarrow} y^{\prime \prime} \wedge u^{\prime \prime} \stackrel{m}{\longrightarrow} z^{\prime \prime} \wedge z^{\prime} \stackrel{a^{\prime}}{\longrightarrow} z^{\prime \prime}\right)\right),
\end{aligned}
$$

$\phi_{5}(u)$ is the formula

$$
\begin{aligned}
& \forall x^{\prime}, y^{\prime}, z^{\prime}, u^{\prime}, x^{\prime \prime}, y^{\prime \prime} \bullet \\
& \bigwedge_{a^{\prime}, b^{\prime} \in \mathrm{A}, a^{\prime} \mid b^{\prime} \neq \delta}\left(u \stackrel{i}{\rightarrow} u^{\prime} \wedge u^{\prime} \stackrel{a^{\prime}}{\rightarrow} x^{\prime \prime} \wedge x^{\prime} \wedge u^{\prime} \stackrel{r}{\rightarrow} y^{\prime} \wedge y^{\prime \prime} \stackrel{m}{\longrightarrow} z^{\prime} \wedge\right. \\
& \exists u^{\prime \prime}, z^{\prime \prime} \bullet \\
& \quad\left(u^{\prime} \stackrel{i}{\rightarrow} u^{\prime \prime} \wedge u^{\prime \prime} \stackrel{l}{\rightarrow} x^{\prime \prime} \wedge u^{\prime \prime} \stackrel{r}{\rightarrow} y^{\prime \prime} \wedge u^{\prime \prime} \stackrel{m}{\longrightarrow} z^{\prime \prime} \wedge\right. \\
& \left.\left.z^{\prime} \stackrel{a^{\prime} \mid b^{\prime}}{\longrightarrow} z^{\prime \prime}\right)\right),
\end{aligned}
$$

$\phi_{6}(u)$ is the formula

$$
\begin{aligned}
& \forall x^{\prime}, y^{\prime}, z^{\prime}, u^{\prime} \bullet \\
& \bigwedge_{a^{\prime} \in \mathrm{A}}\left(u \stackrel{i}{\rightarrow} x^{\prime} \stackrel{a^{\prime}}{\longrightarrow} \vee \sqrt{\longrightarrow} \Rightarrow u^{\prime} \stackrel{l}{\rightarrow} x^{\prime} \wedge z^{\prime} \stackrel{a^{\prime}}{\longrightarrow} y^{\prime}\right),
\end{aligned}
$$

$\phi_{7}(u)$ is the formula

$$
\begin{aligned}
& \forall x^{\prime}, y^{\prime}, z^{\prime}, u^{\prime} \\
& \quad \bigwedge_{a^{\prime} \in \mathrm{A}}\left(u \stackrel{i}{\rightarrow} y^{\prime} \stackrel{a^{\prime}}{\longrightarrow} \vee \sqrt{\rightarrow} \Rightarrow u^{\prime} \stackrel{l}{\rightarrow} x^{\prime} \wedge z^{\prime} \stackrel{a^{\prime}}{\longrightarrow} x^{\prime}\right),
\end{aligned}
$$

$\phi_{8}(u)$ is the formula

$$
\begin{aligned}
& \bigwedge_{a^{\prime}, b^{\prime} \in \mathrm{A}, a^{\prime} \mid b^{\prime} \neq \delta}\left(u \stackrel{i}{\rightarrow} u^{\prime} \wedge u^{\prime} \stackrel{a^{\prime}}{\longrightarrow} x^{\prime \prime} \wedge x^{\prime} \wedge u^{\prime} \stackrel{r}{\rightarrow} y^{\prime} \wedge u^{\prime} \stackrel{m}{\longrightarrow} \sqrt{\longrightarrow} z^{\prime} \wedge z^{\prime} \stackrel{a^{\prime} \mid b^{\prime}}{\longrightarrow} x^{\prime \prime}\right),
\end{aligned}
$$

$\phi_{9}(u)$ is the formula

$$
\begin{aligned}
& \bigwedge_{a^{\prime}, b^{\prime} \in \mathrm{A}, a^{\prime} \mid b^{\prime} \neq \delta}\left(u \stackrel{i}{\rightarrow} x^{\prime} u^{\prime} \wedge u^{\prime} \stackrel{l}{\longrightarrow} \sqrt{\rightarrow} \wedge x^{\prime} \wedge y^{\prime} \stackrel{b^{\prime}}{\longrightarrow} y^{\prime \prime} \Rightarrow u^{\prime} y^{\prime} \stackrel{a^{\prime} \mid b^{\prime}}{\longrightarrow} y^{\prime \prime}\right), \stackrel{m}{\longrightarrow} z^{\prime} \wedge
\end{aligned}
$$

$\phi_{10}(u)$ is the formula

$$
\begin{aligned}
& \bigwedge_{x^{\prime}, y^{\prime}, z^{\prime}, u^{\prime}} \\
& \bigwedge_{a^{\prime}, b^{\prime} \in \mathrm{A}, a^{\prime} \mid b^{\prime} \neq \delta}\left(u \stackrel{i}{\rightarrow} x^{\prime} \stackrel{\stackrel{a^{\prime}}{\longrightarrow}}{\longrightarrow} \wedge \wedge u^{\prime} \stackrel{l}{\rightarrow} x^{\prime} \wedge u^{\prime} \stackrel{b^{\prime}}{\longrightarrow} \sqrt{\longrightarrow} y^{\prime} \wedge z^{\prime} \stackrel{a^{\prime} \mid b^{\prime}}{\longrightarrow} \sqrt{ }\right),
\end{aligned}
$$


$\phi_{11}(u)$ is the formula

$$
\begin{aligned}
& \forall x^{\prime}, y^{\prime}, z^{\prime}, u^{\prime}, z^{\prime \prime} \bullet \\
& \bigwedge\left(u \stackrel{i}{\rightarrow} u^{\prime} \wedge u^{\prime} \stackrel{l}{\rightarrow} x^{\prime} \wedge u^{\prime} \stackrel{r}{\rightarrow} y^{\prime} \wedge u^{\prime} \stackrel{m}{\longrightarrow} z^{\prime} \wedge z^{\prime} \stackrel{a^{\prime}}{\longrightarrow} z^{\prime \prime} \Rightarrow\right. \\
& a^{\prime} \in \mathrm{A} \exists x^{\prime \prime}, u^{\prime \prime} \bullet \\
& \left(u^{\prime} \stackrel{i}{\rightarrow} u^{\prime \prime} \wedge u^{\prime \prime} \stackrel{l}{\rightarrow} x^{\prime \prime} \wedge u^{\prime \prime} \stackrel{r}{\longrightarrow} y^{\prime} \wedge u^{\prime \prime} \stackrel{m}{\longrightarrow} z^{\prime \prime} \wedge x^{\prime} \stackrel{a^{\prime}}{\longrightarrow} x^{\prime \prime}\right) \vee \\
& \exists y^{\prime \prime}, u^{\prime \prime} \bullet \\
& \left(u^{\prime} \stackrel{i}{\rightarrow} u^{\prime \prime} \wedge u^{\prime \prime} \stackrel{l}{\rightarrow} x^{\prime} \wedge u^{\prime \prime} \stackrel{r}{\longrightarrow} y^{\prime \prime} \wedge u^{\prime \prime} \stackrel{m}{\longrightarrow} z^{\prime \prime} \wedge y^{\prime} \stackrel{a^{\prime}}{\longrightarrow} y^{\prime \prime}\right) \vee \\
& \exists x^{\prime \prime}, y^{\prime \prime}, u^{\prime \prime} \bullet \\
& \left(u^{\prime} \stackrel{i}{\rightarrow} u^{\prime \prime} \wedge u^{\prime \prime} \stackrel{l}{\rightarrow} x^{\prime \prime} \wedge u^{\prime \prime} \stackrel{r}{\rightarrow} y^{\prime \prime} \wedge u^{\prime \prime} \stackrel{m}{\longrightarrow} z^{\prime \prime} \wedge\right. \\
& \left.\bigvee_{\mathrm{A}, a^{\prime}=b^{\prime} \mid c^{\prime}}\left(x^{\prime} \stackrel{b^{\prime}}{\longrightarrow} x^{\prime \prime} \wedge y^{\prime} \stackrel{c^{\prime}}{\longrightarrow} y^{\prime \prime}\right)\right) \vee \\
& \left(x^{\prime} \stackrel{a^{\prime}}{\longrightarrow} \vee \wedge z^{\prime \prime}=y^{\prime}\right) \vee\left(y^{\prime} \stackrel{a^{\prime}}{\longrightarrow} \vee \wedge z^{\prime \prime}=x^{\prime}\right) \vee \\
& \bigvee\left(x^{\prime} \stackrel{b^{\prime}}{\longrightarrow} z^{\prime \prime} \wedge y^{\prime} \stackrel{c^{\prime}}{\longrightarrow} \sqrt{ }\right) \vee \\
& b^{\prime}, c^{\prime} \in \mathrm{A}, a^{\prime}=b^{\prime} \mid c^{\prime} \\
& \left.\bigvee\left(x^{\prime} \stackrel{b^{\prime}}{\longrightarrow} \vee \wedge y^{\prime} \stackrel{c^{\prime}}{\longrightarrow} z^{\prime \prime}\right)\right) \wedge \\
& b^{\prime}, c^{\prime} \in \mathrm{A}, a^{\prime}=b^{\prime} \mid c^{\prime} \\
& \forall x^{\prime}, y^{\prime}, z^{\prime}, u^{\prime} \bullet \\
& \bigwedge_{a^{\prime} \in \mathrm{A}}\left(u \stackrel{i}{\rightarrow} u^{\prime} \wedge u^{\prime} \stackrel{l}{\rightarrow} x^{\prime} \wedge u^{\prime} \stackrel{r}{\rightarrow} y^{\prime} \wedge u^{\prime} \stackrel{m}{\longrightarrow} z^{\prime} \wedge z^{\prime} \stackrel{a^{\prime}}{\longrightarrow} \vee \Rightarrow\right. \\
& \left.\bigvee_{b^{\prime}, c^{\prime} \in \mathrm{A}, a^{\prime}=b^{\prime} \mid c^{\prime}}\left(x^{\prime} \stackrel{b^{\prime}}{\longrightarrow} \vee \wedge y^{\prime} \stackrel{c^{\prime}}{\longrightarrow} \sqrt{ }\right)\right) .
\end{aligned}
$$

Formula $\phi_{1}$ expresses that each process reachable by $i$-steps from $u$ relates a process reachable from $x$ and a process reachable from $y$ to a process reachable from $z$. Formula $\phi_{2}$ expresses that $u$ relates $x$ and $y$ to $z$. Formulas $\phi_{3}-\phi_{10}$ express that, if $x^{\prime}$ and $y^{\prime}$ are related to $z^{\prime}$, then $z^{\prime}$ is capable of behaving as $x^{\prime} \| y^{\prime}$. Formula $\phi_{11}$ expresses that, if $x^{\prime}$ and $y^{\prime}$ are related to $z^{\prime}$, then $x^{\prime} \| y^{\prime}$ is capable of behaving as $z^{\prime}$. In other words, the relation $R$ defined by

$$
\begin{aligned}
& R\left(w^{\prime}, z^{\prime}\right) \Leftrightarrow \\
& \quad \exists u^{\prime}, x^{\prime}, y^{\prime} \bullet\left(w^{\prime}=x^{\prime} \| y^{\prime} \wedge u \stackrel{i}{\rightarrow} u^{\prime} \wedge u^{\prime} \stackrel{l}{\rightarrow} x^{\prime} \wedge u^{\prime} \stackrel{r}{\rightarrow} y^{\prime} \wedge u^{\prime} \stackrel{m}{\longrightarrow} z^{\prime}\right)
\end{aligned}
$$

is a bisimulation witnessing the bisimilarity of $x \| y$ and $z$.

The formula $x \| y=z \Leftrightarrow \exists u \bullet \mathrm{P}_{\|}^{\prime}(x, y, z, u)$ is only admissible as an explicit definition of $\|$ if $\exists ! z \bullet \exists u \bullet \mathrm{P}_{\|}^{\prime}(x, y, z, u)$ is derivable. This admissibility condition for $\|$ can be split into an existence condition $\exists z \bullet \exists u \bullet \mathrm{P}_{\|}^{\prime}(x, y, z, u)$ and a uniqueness condition $\exists u \bullet \mathrm{P}_{\|}^{\prime}(x, y, z, u) \wedge \exists u \bullet \mathrm{P}_{\|}^{\prime}(x, y, \bar{z}, u) \Rightarrow z=\bar{z}$. The uniqueness condition for $\|$ is derivable in $\mathrm{BPA}_{\delta \mathrm{rr}}^{\mathrm{fo}}$.

Proposition 16.1 (Uniqueness for Parallel Composition). We have $\mathrm{BPA}_{\delta \mathrm{rr}}^{\mathrm{fo}} \vdash \exists u \bullet \mathrm{P}_{\|}^{\prime}(x, y, z, u) \wedge \exists u \bullet \mathrm{P}_{\|}^{\prime}(x, y, \bar{z}, u) \Rightarrow z=\bar{z}$. 
Proof. Assume $\mathrm{P}_{\|}^{\prime}(x, y, z, u)$ and $\mathrm{P}_{\|}^{\prime}(x, y, \bar{z}, \bar{u})$. Then we derive $z=\bar{z}$ by applying the bisimulation axiom schema BS, taking the following formula for $\phi$ :

$$
\begin{aligned}
& z \rightarrow z^{\prime} \wedge \bar{z} \rightarrow \bar{z}^{\prime} \wedge \\
& \left(z^{\prime}=\bar{z}^{\prime} \vee\right. \\
& \exists x^{\prime}, y^{\prime}, u^{\prime}, \bar{u}^{\prime} \bullet\left(x \rightarrow x^{\prime} \wedge y \rightarrow y^{\prime} \wedge u \stackrel{i}{\rightarrow} u^{\prime} \wedge \bar{u} \stackrel{i}{\rightarrow} \bar{u}^{\prime} \wedge u^{\prime} \stackrel{m}{\rightarrow} z^{\prime} \wedge\right. \\
& \left.\left.\quad \bar{u}^{\prime} \stackrel{m}{\rightarrow} \bar{z}^{\prime} \wedge u^{\prime} \stackrel{l}{\rightarrow} x^{\prime} \wedge \bar{u}^{\prime} \stackrel{l}{\rightarrow} x^{\prime} \wedge u^{\prime} \stackrel{r}{\rightarrow} y^{\prime} \wedge \bar{u}^{\prime} \stackrel{r}{\rightarrow} y^{\prime}\right)\right) .
\end{aligned}
$$

We will come back to the existence condition for $\|$ later on.

As mentioned in Section 13, left merge and communication merge are the same as parallel composition except that the actions that can be performed at the start are restricted. As a consequence, the explicit definitions of the left merge operator and the communication merge operator can be formulated as $x \llbracket y=z \Leftrightarrow \exists u \bullet \mathrm{P}_{\Perp}^{\prime}(x, y, z, u)$ and $x \mid y=z \Leftrightarrow \exists u \bullet \mathrm{P}_{\mid}^{\prime}(x, y, z, u)$, where the formulas for which $\mathrm{P}_{\mathbb{U}}^{\prime}(x, y, z, u)$ and $\mathrm{P}_{\mid}^{\prime}(x, y, z, u)$ stand are simply obtained from the formula for which $\mathrm{P}_{\|}^{\prime}(x, y, z, u)$ stands by replacing at appropriate places $u \stackrel{i}{\rightarrow} u^{\prime}$ by $u \stackrel{i}{\rightarrow} u^{\prime} \wedge \neg u=u^{\prime}$. We refrain from giving the precise formulas for which $\mathrm{P}_{\Perp}^{\prime}(x, y, z, u)$ and $\mathrm{P}_{\mid}^{\prime}(x, y, z, u)$ stand. We mention that the uniqueness conditions for $\llbracket$ and $\mid$ are derivable in $\mathrm{BPA}_{\delta \mathrm{rr}}^{\mathrm{fo}}$.

Next, we consider the explicit definition of the encapsulation operators. As in the case of parallel composition, we begin by introducing the abbreviation $\mathrm{P}_{\partial_{H}}^{\prime}(x, y, u)$, which enables us to formulate the explicit definition of $\partial_{H}$ as $\partial_{H}(x)=y \Leftrightarrow \exists u \bullet \mathrm{P}_{\partial_{H}}^{\prime}(x, y, u)$. We fix different actions $i, l$ and $e$. We use $\mathrm{P}_{\partial_{H}}^{\prime}(x, y, u)$ as an abbreviation of the following formula of $\mathcal{L}\left(\mathrm{BPA}_{\delta \mathrm{r}}^{\mathrm{fo}}\right)$ :

$$
\phi_{1}(x, y, u) \wedge \phi_{2}(x, y, u) \wedge \phi_{3}(u) \wedge \phi_{4}(u) \wedge \phi_{5}(u) ;
$$

where:

$\phi_{1}(x, y, u)$ is the formula

$$
\begin{aligned}
& \forall u^{\prime} \bullet\left(u \stackrel{i}{\rightarrow} u^{\prime} \Rightarrow\right. \\
& \quad \exists ! x^{\prime}, y^{\prime} \bullet\left(x \rightarrow x^{\prime} \wedge y \rightarrow y^{\prime} \Rightarrow u^{\prime} \stackrel{l}{\rightarrow} x^{\prime} \wedge u^{\prime} \stackrel{e}{\rightarrow} y^{\prime}\right) \wedge \\
& \left.\bigwedge_{a^{\prime} \in \mathrm{A}, a^{\prime} \neq i, l, e} \neg \exists v^{\prime} \bullet u^{\prime} \stackrel{a^{\prime}}{\longrightarrow} v^{\prime}\right),
\end{aligned}
$$

$\phi_{2}(x, y, u)$ is the formula

$$
u \stackrel{l}{\rightarrow} x \wedge u \stackrel{e}{\rightarrow} y,
$$

$\phi_{3}(u)$ is the formula

$$
\begin{aligned}
& \forall x^{\prime}, y^{\prime}, u^{\prime}, x^{\prime \prime} \bullet \\
& \bigwedge_{a^{\prime} \in \mathrm{A} \backslash H}\left(u \stackrel{i}{\rightarrow} u^{\prime} \wedge u^{\prime \prime}, y^{\prime \prime} \bullet\left(u^{\prime} \stackrel{l}{\rightarrow} x^{\prime} \wedge u^{\prime} \stackrel{e}{\rightarrow} u^{\prime \prime} \wedge u^{\prime \prime} \stackrel{l}{\rightarrow} x^{\prime \prime} \wedge x^{\prime} \stackrel{a^{\prime}}{\rightarrow} x^{\prime \prime} \stackrel{e}{\rightarrow} y^{\prime \prime} \wedge y^{\prime} \stackrel{a^{\prime}}{\rightarrow} y^{\prime \prime}\right)\right),
\end{aligned}
$$


Table 11. Existence Conditions

\begin{tabular}{ll}
\hline$\exists z \cdot \exists u \cdot \mathrm{P}_{\|}^{\prime}(x, y, z, u)$ & $\mathrm{X} 1$ \\
$\exists z \bullet \exists u \cdot \mathrm{P}_{\Perp}^{\prime}(x, y, z, u)$ & $\mathrm{X} 2$ \\
$\exists z \bullet \exists u \bullet \mathrm{P}_{\mid}^{\prime}(x, y, z, u)$ & $\mathrm{X} 3$ \\
$\exists y \bullet \exists u \bullet \mathrm{P}_{\partial_{H}}^{\prime}(x, y, u)$ & $\mathrm{X} 4$ \\
\hline
\end{tabular}

$\phi_{4}(u)$ is the formula

$$
\forall x^{\prime}, y^{\prime}, u^{\prime} \bullet \bigwedge_{a^{\prime} \in \mathrm{A} \backslash H}\left(u \stackrel{i}{\rightarrow} u^{\prime} \wedge u^{\prime} \stackrel{l}{\rightarrow} x^{\prime} \wedge u^{\prime} \stackrel{e}{\rightarrow} y^{\prime} \wedge x^{\prime} \stackrel{a^{\prime}}{\longrightarrow} \sqrt{ } \Rightarrow y^{\prime} \stackrel{a^{\prime}}{\rightarrow} \sqrt{ }\right),
$$

$\phi_{5}(u)$ is the formula

$$
\begin{aligned}
& \forall x^{\prime}, y^{\prime}, u^{\prime}, y^{\prime \prime} \bullet \\
& \bigwedge_{a^{\prime} \in \mathrm{A}}\left(u \stackrel{i}{\rightarrow} x^{\prime \prime}, u^{\prime \prime} \wedge u^{\prime} \stackrel{l}{\rightarrow} x^{\prime} \wedge u^{\prime} \stackrel{e}{\rightarrow} y^{\prime} \wedge y^{\prime} \stackrel{a^{\prime}}{\rightarrow} y^{\prime \prime} \Rightarrow\right. \\
& \left.\quad\left(u^{\prime} \stackrel{i}{\rightarrow} u^{\prime \prime} \wedge u^{\prime \prime} \stackrel{l}{\rightarrow} x^{\prime \prime} \wedge u^{\prime \prime} \stackrel{e}{\rightarrow} y^{\prime \prime} \wedge \bigvee_{b^{\prime} \in \mathrm{A} \backslash H, a^{\prime}=b^{\prime}} x^{\prime} \stackrel{b^{\prime}}{\rightarrow} x^{\prime \prime}\right)\right) \wedge \\
& \forall x^{\prime}, y^{\prime}, u^{\prime} \bullet \\
& \bigwedge_{a^{\prime} \in \mathrm{A}}\left(u \stackrel{i}{\rightarrow} u^{\prime} \wedge u^{\prime} \stackrel{l}{\rightarrow} x^{\prime} \wedge u^{\prime} \stackrel{e}{\rightarrow} y^{\prime} \wedge y^{\prime} \stackrel{a^{\prime}}{\rightarrow} \sqrt{ } \Rightarrow \underset{b^{\prime} \in \mathrm{A} \backslash H, a^{\prime}=b^{\prime}}{\bigvee^{\rightarrow}} \stackrel{b^{\prime}}{\rightarrow}\right) .
\end{aligned}
$$

The uniqueness condition for $\partial_{H}$ is derivable in $\mathrm{BPA}_{\delta \mathrm{rr}}^{\mathrm{fo}}$.

Proposition 16.2 (Uniqueness for Encapsulation). We have $\mathrm{BPA}_{\delta \mathrm{rr}}^{\text {fo }} \vdash$ $\exists u \bullet \mathrm{P}_{\partial_{H}}^{\prime}(x, y, u) \wedge \exists u \bullet \mathrm{P}_{\partial_{H}}^{\prime}(x, \bar{y}, u) \Rightarrow y=\bar{y}$.

Proof. The proof follows the same line as to the proof of Proposition 16.1.

The formulas of $\mathcal{L}\left(\mathrm{BPA}_{\delta \mathrm{rr}}^{\text {fo }}\right)$ that are given in Table 11 are existence conditions for $\|\|,, \mid$ and $\partial_{H}$. We write $\mathrm{X}$ for this set of formulas. X4 is actually a schema with an instance for each $H \subseteq \mathrm{A}$. The existence conditions from Table 11 are valid in the full bisimulation models $\mathfrak{P}_{\kappa}^{\text {rr }}\left(\kappa \geq \aleph_{0}\right)$. It is unknown to us whether they are derivable from $\mathrm{BPA}_{\delta \mathrm{rr}}^{\text {fo }}$.

Theorem 16.1 (Interpretation of $\mathbf{A C P}^{\text {fo }}$ in $\mathbf{B P A} \mathbf{A r r}_{\delta \mathrm{rr}}^{\text {) }}$. The following is an interpretation of $\mathrm{ACP}^{\text {fo }}$ in $\mathrm{BPA}_{\delta \mathrm{rr}}^{\text {fo }} \cup \mathrm{X}$ :

$$
\begin{aligned}
& \mathfrak{d}(x) \Leftrightarrow x=x, \\
& x \| y=z \Leftrightarrow \exists u \bullet \mathrm{P}_{\|}^{\prime}(x, y, z, u), \\
& x \| y=z \Leftrightarrow \exists u \bullet \mathrm{P}_{\Perp}^{\prime}(x, y, z, u), \\
& x \mid y=z \Leftrightarrow \exists u \bullet \mathrm{P}_{\mid}^{\prime}(x, y, z, u), \\
& \partial_{H}(x)=y \Leftrightarrow \exists u \bullet \mathrm{P}_{\partial_{H}}^{\prime}(x, y, u) \quad \text { for each } H \subseteq \mathrm{A} .
\end{aligned}
$$


Proof. Because $\mathfrak{d}(x) \Leftrightarrow x=x$, the first two conditions made in the definition of interpretation are trivially fulfilled. Because $\mathfrak{d}(x) \Leftrightarrow x=x$, the third condition becomes

$$
\mathrm{BPA}_{\delta \mathrm{rr}}^{\mathrm{fo}} \cup \mathrm{X} \cup \mathrm{E} \vdash \phi \text { for each axiom } \phi \text { of } \mathrm{ACP}^{\mathrm{fo}},
$$

where $\mathrm{E}$ is the set of explicit definitions given above. For each axiom $\phi$ of $\mathrm{BPA}_{\delta}^{\mathrm{fo}}$, we immediately have $\mathrm{BPA}_{\delta \mathrm{rr}}^{\mathrm{fo}} \cup \mathrm{X} \cup \mathrm{E} \vdash \phi$. Hence, it is sufficient to establish $\mathrm{BPA}_{\delta \mathrm{rr}}^{\mathrm{fo}} \cup \mathrm{X} \cup \mathrm{E} \vdash \phi$ only for each axiom $\phi$ of $\mathrm{ACP}^{\text {fo }}$ that is not an axiom of $\mathrm{BPA}_{\delta}^{\mathrm{fo}}$.

All axioms in question are atomic formulas of $\mathcal{L}\left(\mathrm{BPA}_{\delta \mathrm{rr}}^{\mathrm{fo}} \cup \mathrm{E}\right)$. Each atomic formula $\phi$ of $\mathcal{L}\left(\mathrm{BPA}_{\delta \mathrm{rr}}^{\mathrm{fo}} \cup \mathrm{E}\right)$ is equivalent in $\mathrm{BPA}_{\delta \mathrm{rr}}^{\mathrm{fo}} \cup \mathrm{X} \cup \mathrm{E}$ to an existential formula $\phi^{\prime}$ of $\mathcal{L}\left(\mathrm{BPA}_{\delta \mathrm{rr}}^{\text {fo }} \cup \mathrm{E}\right)$ in which no other terms occur than terms of $\mathcal{L}\left(\mathrm{BPA}_{\delta \mathrm{rr}}^{\text {fo }}\right)$ and terms $t_{1} \| t_{2}, t_{1} \Perp t_{2}, t_{1} \mid t_{2}$ and $\partial_{H}\left(t_{1}\right)$ of which the subterms $t_{1}$ and $t_{2}$ are terms of $\mathcal{L}\left(\mathrm{BPA}_{\delta \mathrm{rr}}^{\mathrm{fo}}\right)$ (see e.g. [24]). Because $\mathrm{E}$ contains the explicit definitions for $\|$,$\| ,$ $\mid$ and $\partial_{H}$, this existential formula $\phi^{\prime}$ is equivalent in $\mathrm{BPA}_{\delta \mathrm{rr}}^{\mathrm{fo}} \cup \mathrm{X} \cup \mathrm{E}$ to a formula $\phi^{\prime \prime}$ of $\mathcal{L}\left(\mathrm{BPA}_{\delta \mathrm{rr}}^{\mathrm{fo}}\right)$. Because definitional extensions are conservative extensions (see e.g. [28]), $\mathrm{BPA}_{\delta \mathrm{rr}}^{\mathrm{fo}} \cup \mathrm{X} \cup \mathrm{E} \vdash \phi^{\prime \prime}$ iff $\mathrm{BPA}_{\delta \mathrm{rr}}^{\mathrm{fo}} \cup \mathrm{X} \vdash \phi^{\prime \prime}$. This suggests the following three-steps approach to establish that $\mathrm{BPA}_{\delta \mathrm{rr}}^{\mathrm{fo}} \cup \mathrm{X} \cup \mathrm{E} \vdash \phi$ :

1. eliminate from $\phi$ all nested terms other than terms of $\mathcal{L}\left(\mathrm{BPA}_{\delta \mathrm{rr}}^{\mathrm{fo}}\right)$, resulting in $\phi^{\prime}$;

2. eliminate from $\phi^{\prime}$ all atomic formulas in which $\|, \downarrow, \mid$ or $\partial_{H}$ occur, resulting in $\phi^{\prime \prime}$;

3. derive $\phi^{\prime \prime}$ from $\mathrm{BPA}_{\delta \mathrm{rr}}^{\mathrm{fo}} \cup \mathrm{X}$.

For each axiom of $\mathrm{ACP}^{\text {fo }}$ that is not an axiom of $\mathrm{BPA}_{\delta}^{\text {fo }}$, the first two steps are short and simple. The last step is generally straightforward, but tedious. We outline the proof for axioms CM3 and CM4.

The first two steps result for CM3 in the formula

$$
\exists z \bullet\left(\exists u \bullet \mathrm{P}_{\llbracket}^{\prime}(a \cdot x, y, a \cdot z, u) \wedge \exists u^{\prime} \bullet \mathrm{P}_{\|}^{\prime}\left(x, y, z, u^{\prime}\right)\right)
$$

and for CM4 in the formula

$$
\begin{aligned}
\exists v, w \bullet & \left(\exists u \bullet \mathrm{P}_{\llbracket}^{\prime}(x+y, z, v+w, u) \wedge\right. \\
& \left.\exists u^{\prime} \bullet \mathrm{P}_{\llbracket}^{\prime}\left(x, z, v, u^{\prime}\right) \wedge \exists u^{\prime \prime} \bullet \mathrm{P}_{\llbracket}^{\prime}\left(y, z, w, u^{\prime \prime}\right)\right) .
\end{aligned}
$$

The last step for CM3 goes as follows. First of all, it follows from X that $\exists z \bullet$ $\exists u^{\prime} \bullet \mathrm{P}_{\|}^{\prime}\left(x, y, z, u^{\prime}\right)$. Therefore, it is sufficient to show that $\exists u^{\prime} \bullet \mathrm{P}_{\|}^{\prime}\left(x, y, z, u^{\prime}\right) \Rightarrow$ $\exists u \bullet \mathrm{P}_{\|}^{\prime}(a \cdot x, y, a \cdot z, u)$. This is done as follows. Assume $\mathrm{P}_{\|}^{\prime}\left(x, y, z, u^{\prime}\right)$. Take $l \cdot(a \cdot x)+r \cdot y+m \cdot(a \cdot z)+i \cdot u^{\prime}$ for $u$. Then $\mathrm{P}_{\|}^{\prime}(a \cdot x, y, a \cdot z, u)$ is easily derived.

The last step for CM4 follows essentially the same line as the last step for CM3. However, there are two complications in the construction of $u$ from $u^{\prime}$ and $u^{\prime \prime}$. The first complication is that four cases have to be distinguished according to the reachability of $x$ from $x$ in one or more steps and the reachability of $y$ from $y$ in one or more steps. The second complication is that $u$ has to be constructed from subprocesses of $u^{\prime}$ and $u^{\prime \prime}$ instead of $u^{\prime}$ and $u^{\prime \prime}$ themselves. 
Thus, although the construction of $u$ is rather straightforward, it becomes very tedious to express it in $\mathcal{L}\left(\mathrm{BPA}_{\delta \mathrm{rr}}^{\mathrm{fo}}\right)$ and to derive $\mathrm{P}_{\|}^{\prime}(x+y, z, v+w, u)$. We refrain from outlining the last step for $\mathrm{CM} 4$ further.

The proofs for CM2, CM5-CM7 and D4 are similar to the proof for CM3. The proofs for CM1, CM8-CM9 and D3 are similar to the proof for CM4. The proofs for $\mathrm{C} 1-\mathrm{C} 3$ are easy.

\section{Concluding Remarks}

In this paper, we build on earlier work on ACP. The algebraic theory ACP was first presented in [12] and RDP, RSP and AIP were first formulated in [14]. Moreover, the full bisimulation models are basically the graph models of ACP, which are most extensively described in [5]. In this paper, we extend ACP to a first-order theory and look into that theory from classical model theory. Some open problems that arise from this work are:

- What are the relations between RDP, RSP (Table 2), B, R (Table 3) and AIP (Table 4) in the presence of $\mathrm{BPA}_{\delta}^{\text {for }}$ ? In particular, do all models of $\mathrm{BPA}_{\delta}^{\text {fo }}$ extended with $\mathrm{R}$ satisfy $\mathrm{B}$ ?

- Is it derivable from $\mathrm{BPA}_{\delta}^{\mathrm{fo}}$ or a finitary first-order extension thereof, for all pairs of guarded recursive specifications of which the solutions in $\mathfrak{P}_{\aleph_{0}}$ are not identical, that their solutions are not equal?

- Are the restricted reachability predicates $\stackrel{a}{\rightarrow}$ of $\mathrm{BPA}_{\delta \mathrm{rr}}^{\mathrm{fo}}$ definable in terms of $\mathcal{L}\left(\mathrm{BPA}_{\delta}^{\text {fo }}\right)$ in $\mathrm{BPA}_{\delta}^{\text {fo }}$ ?

- Is axiom RR3 (Table 8) derivable from the other axioms of $\mathrm{BPA}_{\delta \mathrm{rr}}^{\mathrm{fo}}$ ?

- Are the existence conditions for $\|, \mathbb{L}, \mid$ and $\partial_{H}$ (Table 11) derivable from $\mathrm{BPA}_{\delta \mathrm{rr}}^{\mathrm{fo}}$ ?

To the best of our knowledge there is no related work. Many options for future work remain. We mention:

- Development of extensions of $\mathrm{ACP}^{\text {fo }}$ with additional operators, such as the iteration operators from $[9,10,19]$.

- Development of first-order extensions of variants of ACP with timing, such as the ones from $[1,3,6]$.

- Re-development of the $\alpha / \beta$-calculus [4] in the setting of $\mathrm{ACP}^{\mathrm{fo}}$.

- Further analysis of the relation between external and internal versions of predicates on processes.

- Further investigations into interpretation of existing process algebras in $\mathrm{ACP}^{\text {fo }}$.

- Investigations into interpretation of other related algebraic theories, such as the network algebra from [18], in $\mathrm{ACP}^{\mathrm{fo}}$.

- Exploration of the strong and weak points of $\mathrm{ACP}^{\text {fo }}$ for process specification and verification. 


\section{References}

1. J. C. M. Baeten and J. A. Bergstra. Real time process algebra. Formal Aspects of Computing, 3(2):142-188, 1991.

2. J. C. M. Baeten and J. A. Bergstra. On sequential composition, action prefixes and process prefix. Formal Aspects of Computing, 6:250-268, 1994.

3. J. C. M. Baeten and J. A. Bergstra. Discrete time process algebra. Formal Aspects of Computing, 8(2):188-208, 1996.

4. J. C. M. Baeten, J. A. Bergstra, and J. W. Klop. Conditional axioms and $\alpha / \beta$ calculus in process algebra. In M. Wirsing, editor, Formal Description of Programming Concepts III, pages 53-75. North-Holland, 1987.

5. J. C. M. Baeten, J. A. Bergstra, and J. W. Klop. On the consistency of Koomen's fair abstraction rule. Theoretical Computer Science, 51(1/2):129-176, 1987.

6. J. C. M. Baeten and C. A. Middelburg. Process Algebra with Timing. Monographs in Theoretical Computer Science, An EATCS Series. Springer-Verlag, Berlin, 2002.

7. J. C. M. Baeten and W. P. Weijland. Process Algebra, volume 18 of Cambridge Tracts in Theoretical Computer Science. Cambridge University Press, Cambridge, 1990.

8. J. A. Bergstra and I. Bethke. Polarized process algebra and program equivalence. In J. C. M. Baeten, J. K. Lenstra, J. Parrow, and G. J. Woeginger, editors, Proceedings 30th ICALP, volume 2719 of Lecture Notes in Computer Science, pages 1-21. Springer-Verlag, 2003.

9. J. A. Bergstra, I. Bethke, and A. Ponse. Process algebra with iteration and nesting. Computer Journal, 37:243-258, 1994.

10. J. A. Bergstra, W. J. Fokkink, and A. Ponse. Process algebra with recursive operations. In J. A. Bergstra, A. Ponse, and S. A. Smolka, editors, Handbook of Process Algebra, pages 333-389. Elsevier, Amsterdam, 2001.

11. J. A. Bergstra and J. W. Klop. The algebra of recursively defined processes and the algebra of regular processes. In J. Paredaens, editor, Proceedings 11th ICALP, volume 172 of Lecture Notes in Computer Science, pages 82-95. Springer-Verlag, 1984.

12. J. A. Bergstra and J. W. Klop. Process algebra for synchronous communication. Information and Control, 60(1/3):109-137, 1984.

13. J. A. Bergstra and J. W. Klop. Algebra of communicating processes. In J. W. de Bakker, M. Hazewinkel, and J. K. Lenstra, editors, Proceedings Mathematics and Computer Science I, volume 1 of CWI Monograph, pages 89-138. North-Holland, 1986.

14. J. A. Bergstra and J. W. Klop. Process algebra: Specification and verification in bisimulation semantics. In M. Hazewinkel, J. K. Lenstra, and L. G. L. T. Meertens, editors, Proceedings Mathematics and Computer Science II, volume 4 of CWI Monograph, pages 61-94. North-Holland, 1986.

15. J. A. Bergstra and J. W. Klop. Process theory based on bisimulation semantics. In J. W. de Bakker, W. P. de Roever, and G. Rozenberg, editors, Linear Time, Branching Time and Partial Order in Logics and Models for Concurrency, volume 354 of Lecture Notes in Computer Science, pages 50-122. Springer-Verlag, 1989.

16. J. A. Bergstra and J. W. Klop. A convergence theorem in process algebra. In J. W. de Bakker and J. J. M. M. Rutten, editors, Ten Years of Concurrency Semantics, pages 164-195. World Scientific, 1992.

17. J. A. Bergstra and M. E. Loots. Program algebra for sequential code. Journal of Logic and Algebraic Programming, 51(2):125-156, 2002. 
18. J. A. Bergstra, C. A. Middelburg, and Gh. Ştefănescu. Network algebra for asynchronous dataflow. International Journal of Computer Mathematics, 65(1/2):5788, 1997.

19. J. A. Bergstra and A. Ponse. Non-regular iterators in process algebra. Theoretical Computer Science, 269:203-229, 2001

20. C. C. Chang and H. J. Keisler. Model Theory, volume 73 of Studies in Logic and the Foundations of Mathematics. Elsevier, Amsterdam, third edition, 1990.

21. W. J. Fokkink. A complete equational axiomatization for prefix iteration. Information Processing Letters, 52(6):333-337, 1994.

22. W. J. Fokkink. Introduction to Process Algebra. Texts in Theoretical Computer Science, An EATCS Series. Springer-Verlag, Berlin, 2000.

23. M. Hennessy and R. Milner. Algebraic laws for non-determinism and concurrency. Journal of the ACM, 32:137-161, 1985.

24. W. A. Hodges. Model Theory, volume 42 of Encyclopedia of Mathematics and Its Applications. Cambridge University Press, Cambridge, 1993.

25. J. E. Hopcroft and J. D. Ullman. Introduction to Automata Theory, Languages and Computation. Addison-Wesley, Reading, MA, 1979.

26. R. Milner. A Calculus of Communicating Systems, volume 92 of Lecture Notes in Computer Science. Springer-Verlag, Berlin, 1980.

27. R. Milner. Communication and Concurrency. Prentice-Hall, Englewood Cliffs, 1989.

28. J. R. Shoenfield. Mathematical Logic. Addison-Wesley Series in Logic. AddisonWesley, Reading, MA, 1967.

29. J. F. A. K. van Benthem and J. A. Bergstra. Logic of transition systems. Journal of Logic, Language and Information, 3:247-283, 1995.

30. J. F. A. K. van Benthem, D. J. N. van Eijck, and V. Stebletsova. Modal logic, transition systems and processes. Journal of Logic and Computation, 4:811-855, 1994. 